WHC-EP-0527-5

UC-630

\title{
Environmental Releases for Calendar Year 1995
}

B. P. Gleckler

Date Published

July 1996

Prepared for the U.S. Department of Energy Office of Environmental Restoration and Waste Management

$\begin{array}{ll}\text { (2) Westinghouse } & \text { P.O Box } 1970 \\ \text { Hanford Company } & \text { Richland, Washington }\end{array}$

Management and Operations Contractor for the

U.S. Department of Energy under Contract DE-AC06-87RL10930

Approved for Public Release 
THIS PAGE INTENTIONAILY LEFT BLANK 


\section{RELEASE AUTHORIZATION}

Document Number:

Document Title:
WHC-EP-0527-5

Environmental Releaseg For Calendar Year 1995

Release Date: $07 / 30 / 96$

This document was reviewed following the procedures described in WHC-CM-3-4 and is:

\section{APPROVED FOR PUBLIC RELEASE}

wHC Information Release Administration specialist:
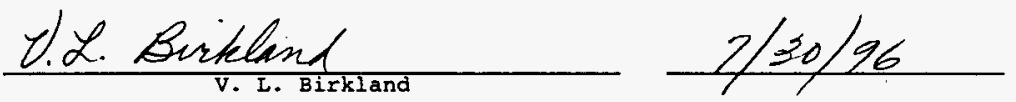
THIS PAGE INTENTJONALLY

LET TSLANK 
WHC-EP-0527-5

\section{APPROVAL PAGE}

Document Title: Environmental Releases for Calendar Year 1995

Prepared by:

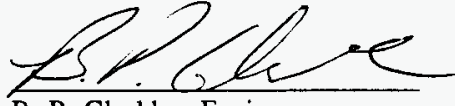

B. P. Gleckler, Engineer

Air \& Water Services Integration

Approved by:

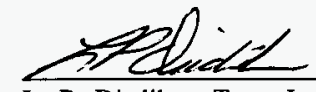

L. P. Diediker, Team Leader

Air \& Water Services Integration

$$
\frac{7 / 3 / / 96}{\text { Date }}
$$

$7-3 /-9$

Date 
WHC-EP-0527-5

This page intentionally left blank. 
WHC-EP-0527-5

\title{
ENVIRONMENTAL RELEASES FOR \\ CALENDAR YEAR 1995
}

\begin{abstract}
This report presents data on radioactive and nonradioactive materials released into the environment during calendar year 1995 from facilities managed by the Westinghouse Hanford Company and facilities monitored by Bechtel Hanford, Incorporated. The Westinghouse Hanford Company provides effluent monitoring services for Bechtel Hanford, Incorporated, which includes release reporting. Both summary and detailed presentations of the environmental releases are provided. When appropriate, comparisons to data from previous years are made.
\end{abstract}


WHC-EP-0527-5

This page intentionally left blank. 


\section{EXECUTIVE SUMMARY}

This report fulfills the annual environmental release reporting requirements of U.S. Department of Energy (DOE) Orders $5484.1^{*}$ and 5400.1.** This report provides supplemental information to the Hanford Site Environmental Report. The Hanford Site Environmental Report provides an update on the environmental status of the entire Hanford Site. The sitewide annual report summarizes the degree of compliance of the Hanford Site with applicable environmental regulations and informs the public about the impact of Hanford operations on the surrounding environment.

Like the Hanford Site Environmental Report, this annual report presents a summary of the environmental releases from facilities managed by the Westinghouse Hanford Company (WHC) and facilities monitored by Bechtel Hanford, Incorporated (BHI). In addition to the summary data, this report also includes detailed data on air emissions, liquid effluents, and hazardous substances released to the environment during calendar year 1995 from these facilities.

*DOE, 1990, Environmental Protection, Safety, and Health Protection Information Reporting Requirements, DOE Order 5484.1, Change 7, U.S. Department of Energy, Washington, D.C.

**DOE, 1990, General Environmental Protection Program, DOE Order 5400.1, Change 1, U.S. Department of Energy, Washington, D.C. 
As part of this executive summary, comprehensive data summaries of air emissions and liquid effluents in 1995 are displayed in Tables ES-1 through ES-5. These tables represent the following:

- Table ES-1. Radionuclide air emissions data (detailed data on emissions are presented in Section 2.0)

- Table ES-2. Data on radioactive liquid effluents discharged to the soil (detailed data on releases from individual waste streams to soil column disposal sites are presented in Section 3.0)

- Table ES-3. Radionuclides discharged to the Columbia River (detailed data are shown in Section 3.0)

- Table ES-4. Nonradioactive air emissions data (detailed information is given in Section 2.0)

- Table ES-5. Total Volumes and Flow Rates of 200/600 Area Liquid Effluents (detailed data are presented in Section 3.0). 
WHC-EP-0527-5

Table ES-1

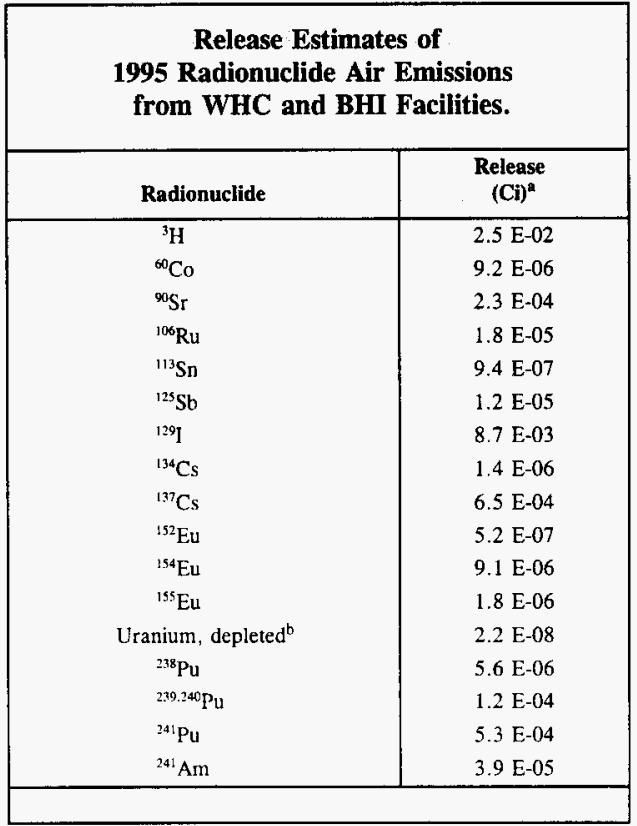

a $1 \mathrm{Ci}=3.7 \mathrm{E}+10$ Becquerel; $\mathrm{ND}=$ not detected (i.e. either the radionuclide was not detected in any sample during the year, or the average of all the measurements for that given radionuclide or type of radioactivity made during the year was below background levels).

b Determined from total alpha measurements. Assumed to be depleted uranium consisting of $63.478 \mathrm{Ci} \%{ }^{238} \mathrm{U}, 0.821 \mathrm{Ci} \%{ }^{235} \mathrm{U}$, and $35.701 \mathrm{Ci} \%$ ${ }^{234} \mathrm{U}\left(99.797 \mathrm{Wt} \%{ }^{238} \mathrm{U}, 0.200 \mathrm{Wt} \%{ }^{235} \mathrm{U}\right.$, and $\left.0.003 \mathrm{Wt} \%{ }^{234} \mathrm{U}\right)$. 
Table ES-2

\begin{tabular}{|c|c|}
\hline \multicolumn{2}{|c|}{$\begin{array}{l}\text { Release Estimates of } \\
1995 \text { Radioactive Liquid Effluents Discharged to Soi } \\
\text { from WHC and BHI Facilities. }\end{array}$} \\
\hline Radionuclide & $\begin{array}{c}\text { Release } \\
\left.(\mathrm{C})^{2}\right)^{2}\end{array}$ \\
\hline $\begin{array}{l}{ }^{3} \mathrm{H} \\
{ }^{60} \mathrm{Co} \\
{ }^{90} \mathrm{Sr} \\
{ }^{90} \mathrm{Tc} \\
{ }^{106} \mathrm{Ru} \\
{ }^{113} \mathrm{Sn} \\
{ }^{125} \mathrm{Sb} \\
{ }^{139} \mathrm{Cs} \\
{ }^{137} \mathrm{Cs} \\
{ }^{152} \mathrm{Eu} \\
{ }^{154} \mathrm{Eu} \\
{ }^{155} \mathrm{Eu} \\
{ }^{\mathrm{Uranium}} \text {, natural }{ }^{\mathrm{b}} \\
{ }^{238} \mathrm{Pu} \\
{ }^{239.240} \mathrm{Pu} \\
{ }^{241} \mathrm{Am}\end{array}$ & $\begin{array}{l}1.5 \mathrm{E}-02 \\
9.5 \mathrm{E}-03 \\
1.2 \mathrm{E}-01^{\mathrm{b}} \\
2.3 \mathrm{E}-04 \\
1.9 \mathrm{E}-01 \\
1.0 \mathrm{E}-01 \\
3.8 \mathrm{E}-03 \\
1.2 \mathrm{E}-03 \\
4.5 \mathrm{E}-02 \\
1.1 \mathrm{E}-03 \\
3.3 \mathrm{E}-02 \\
2.3 \mathrm{E}-02 \\
5.5 \mathrm{E}-04 \\
7.5 \mathrm{E}-04 \\
7.2 \mathrm{E}-03 \\
1.2 \mathrm{E}-03 \\
\end{array}$ \\
\hline
\end{tabular}

a $\quad 1 \mathrm{Ci}=3.7 \mathrm{E}+10$ Becquerel.

b Determined from total alpha measurements. Assumed to be natural uranium. 
WHC-EP-0527-5

Table ES-3

\begin{tabular}{|c|c|}
\hline \multicolumn{2}{|c|}{$\begin{array}{l}\text { Release Estimates of } \\
1995 \text { Radionuclides in Liquid Effluents } \\
\text { Discharged to the Columbia River } \\
\text { from WHC and BHI Facilities. }\end{array}$} \\
\hline Radionuclide & $\begin{array}{c}\text { Release } \\
(\mathrm{Ci})^{\mathrm{a}}\end{array}$ \\
\hline${ }^{3} \mathrm{H}$ & $1.5 \mathrm{E}-01$ \\
\hline${ }^{60} \mathrm{Co}$ & $1.1 \mathrm{E}-04$ \\
\hline${ }^{90} \mathrm{Sr}$ & $2.1 \mathrm{E}-01$ \\
\hline${ }^{106} \mathrm{Ru}$ & ND \\
\hline${ }^{12} \mathrm{Sb}$ & $2.7 \mathrm{E}-04$ \\
\hline${ }^{134} \mathrm{Cs}$ & ND \\
\hline${ }^{i 37} \mathrm{Cs}$ & $2.1 \mathrm{E}-03$ \\
\hline${ }^{154} \mathrm{Eu}$ & $4.1 \mathrm{E}-03$ \\
\hline${ }^{238} \mathrm{Pu}$ & ND \\
\hline${ }^{239: 24: 40} \mathrm{Pu}$ & $4.3 \mathrm{E}-07$ \\
\hline${ }^{241} \mathrm{Am}$ & $1.2 \mathrm{E}-05$ \\
\hline
\end{tabular}

a $\quad 1 \mathrm{Ci}=3.7 \mathrm{E}+10$ Becquerel; $\mathrm{ND}=$ Not Detected. 
WHC-EP-0527-5

Table ES-4

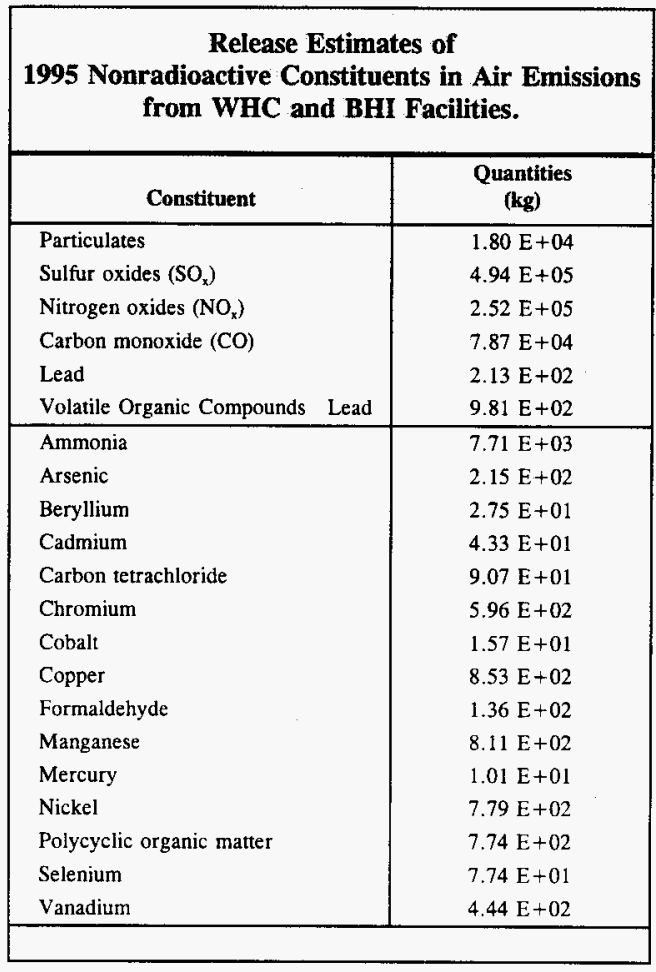


Table ES-5

\begin{tabular}{|c|c|c|c|c|c|c|c|}
\hline \multirow{3}{*}{$\begin{array}{c}\text { Stream } \\
\text { Code }\end{array}$} & \multicolumn{7}{|c|}{$\begin{array}{l}\text { Total Volumes and Flow Rates of Liquid Effluents } \\
\text { Discharged to the } 200 / 600 \text { Area Disposal Sites during } 1994 \text { and } 1995 \\
\text { from WHC and BHI Facilities }{ }^{a} \text {. }\end{array}$} \\
\hline & \multirow{2}{*}{$\begin{array}{l}\text { EDP } \\
\text { Code }\end{array}$} & \multirow[t]{2}{*}{ Effluent Source } & \multirow[t]{2}{*}{ Disposal Site } & \multicolumn{2}{|c|}{$\begin{array}{l}\text { Volume } \\
\text { (L) }\end{array}$} & \multicolumn{2}{|c|}{$\begin{array}{c}\text { Flow Rate } \\
\text { (gpm) }\end{array}$} \\
\hline & & & & 1994 & 1995 & 1994 & 1995 \\
\hline $207-\mathrm{SL}$ & $\mathrm{H} 101$ & 222-S Laboratory Chemical Sewer & $216-5-26$ Crib & $6.3 \mathrm{E}+06$ & $2.4 \mathrm{E}+06$ & 3.2 & 2.8 \\
\hline $207-U$ & H102 & $\mathrm{UO}_{3}$ Plant Waste Water & 216.U.14 Ditch & $1.1 \mathrm{E}+08$ & 0.0 & 55.3 & 0 \\
\hline 2904-ZA & H103 & PFP Cooling Water & $216-\mathrm{Z}-20 \mathrm{Crib}$ & $2.7 \mathbf{E}+07$ & $7.3 \mathrm{E}+06$ & 13.3 & 9.2 \\
\hline $\mathrm{ACW}$ & $\mathrm{H} 108$ & 242-A Evaporator Cooling Water & 216-B-3 Pond & $1.6 \mathrm{E}+09$ & $7.8 \mathrm{E}+08$ & 780 & 1175 \\
\hline ASC & H110 & 242-A Evaporator Steam Condensate & 216-B-3 Pond & $5.2 \mathrm{E}+07$ & $2.3 \mathrm{E}+07$ & 25.9 & 22.9 \\
\hline CA8 & H115 & 241-A Tank Farm Cooling Water & 216-B-3 Pond & $6.5 \mathrm{E}+08$ & $8.3 \mathrm{E}+08$ & 327 & 414 \\
\hline CAR & H116 & 244.AR Vault Cooling Water & 216-B-3 Pond & $8.2 \mathbf{E}+07$ & $4.8 \mathrm{E}+06$ & 41.2 & 2 \\
\hline $\mathrm{CBC}$ & $\mathrm{H} 117$ & B Plant Cooling Water & 216-B-3 Pond & $3.2 \mathrm{E}+09$ & $3.0 \mathrm{E}+09$ & 1608 & 1491 \\
\hline CSL & $\mathrm{H} 118$ & PUREX Chemical Sewer & 216-B-3 Pond & 4.6 $\mathrm{E}+08$ & $1.8 \mathrm{E}+08$ & 233 & 230 \\
\hline CWL & $\mathrm{H} 119$ & PUREX Cooling Water & 216-B-3 Pond & 0.0 & 0.0 & 0 & 0 \\
\hline LWC & H 120 & Laundry Waste Water & 216.W-LC Crib & 0.0 & 0.0 & 0 & 0 \\
\hline $\mathrm{U}-17$ & $\mathrm{H} 126$ & UO, Plant Process Condensate & 216-U-17 Crib & $3.3 \mathrm{E}+0.5$ & 0.0 & 0.2 & 0 \\
\hline
\end{tabular}

a These discharges do not include discharges to the 200 East Area Treated Effluent Disposal Facility, since discharges from this facility meet drinking water standards. Streams CSL, 207-SL, and 2904-ZA no longer discharge to the environment as of May 1995. 
WHC-EP-0527-5

This page intentionally left blank. 


\section{CONTENTS}

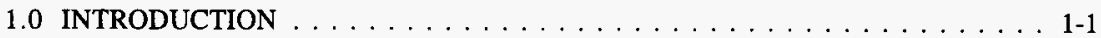

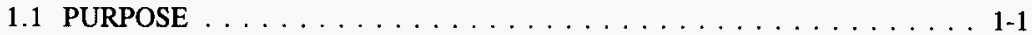

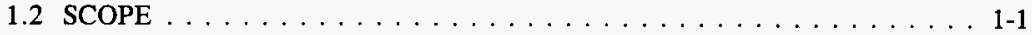

1.3 TYPES AND LOCATIONS OF RELEASES . . . . . . . . . . 1-2

1.4 ENVIRONMENTAL RELEASE LIMITS AND GUIDELINES . . . . . . 1-2

1.4.1 Limits for Radioactive Releases . . . . . . . . . . . . . . . . 1-2

1.4.2 Limits for Nonradioactive Releases . . . . . . . . . . . . . 1-3

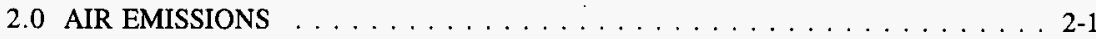

2.1 RADIONUCLIDE AIR EMISSIONS $\ldots \ldots \ldots \ldots \ldots \ldots \ldots \ldots$. . . . . . . .

2.1.1 Filtration of Radionuclide Air Emissions . . . . . . . . . . . 2-1

2.1.2 Radionuclide Air Emissions . . . . . . . . . . . . . . 2-1

2.1.3 Nonradioactive Constituents in Radionuclide Air Emissions . . . . 2-2

2.2 NONRADIOACTIVE AIR EMISSIONS . . . . . . . . . . 2-3

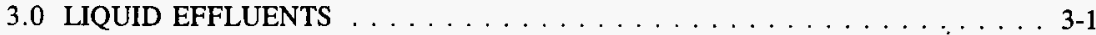

3.1 RADIOACTIVE LIQUID EFFLUENTS . . . . . . . . . . . . 3-1

3.2 NONRADIOACTIVE LIQUID EFFLUENTS $\ldots \ldots \ldots \ldots \ldots$. . . . . . . . .

3.2.1 Sanitary Sewage Discharges to the Soil . . . . . . . . . . . . 3-3

3.2.2 National Pollutant Discharge Elimination System Discharges . . . 3 3-3

3.2.3 Process Water Discharges . . . . . . . . . . . . . 3-3

4.0 HAZARDOUS SUBSTANCE RELEASES $\ldots \ldots \ldots \ldots \ldots \ldots \ldots$. . . . . . . . .

4.1 NONROUTINE RELEASES . . . . . . . . . . . . . . . . 4 4-1

4.2 ROUTINE CONTINUOUS RELEASES . . . . . . . . . . . . . 4-1

5.0 REFERENCES . . . . . . . . . . . . . . . . . 5-1

\section{APPENDICES}

A POINT SOURCES FOR RADIONUCLIDE AIR EMISSIONS $\ldots \ldots \ldots \ldots \ldots$ A-1

B MONITORING PROGRAM FOR AIR EMISSIONS AND LIQUID EFFLUENTS . B-1 


\section{LIST OF FIGURES}

2-1. Historical Effective Dose Equivalents Received by the Maximally Exposed Individual Located Offsite, from Airborne Radionuclide Emissions. . . . . . . . 2-2

2-2. Historical Airborne Effluent Releases of ${ }^{239 / 240} \mathrm{Pu} \ldots \ldots \ldots \ldots \ldots$. . . . . . . . . .

2-3. Historical Airborne Effluent Releases of ${ }^{129} \mathrm{I} \ldots \ldots \ldots$. . . . . . . . . . 2-5

3-1. Historical Liquid Effluent Releases of ${ }^{90} \mathrm{Sr}$ to the Columbia River . . . . . . . . 3-1

\section{LIST OF TABLES}

2-1. Release Estimates of 1995 Radionuclide Air Emissions from WHC and BHI Facilities. . . . . . . . . . . . . . . . . . . . . 2-4

2-2. 1995 Radionuclide Air Emissions Data for Major Point Sources from WHC and BHI Facilities . . . . . . . . . . . . . . . . . . . . 2-7

2-3. 1995 Radionuclide Air Emissions Data for Minor Point Sources from WHC and BHI

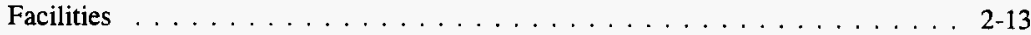

2-4. 1995 Nonradioactive Air Emissions from WHC Facilities . . . . . . . . . 2-22

3-1. 1995 Radionuclide Liquid Effluent Data for Individual Effluent Streams Discharged from WHC and BHI Facilities . . . . . . . . . . . . 3-5

3-2. Sanitary Sewage Discharged in $1995 \ldots \ldots$. . . . . . . . . . . . . 3-10

3-3. National Pollutant Discharge Elimination System (NPDES) Discharge Points . . . 3-11

3-4. Summary of National Pollutant Discharge Elimination System (NPDES) Data for 1995

4-1. Comprehensive Environmental Response, Compensation, and Liability Act of 1980 Continuous Release Summary for Calendar Year 1995 
WHC-EP-0527-5

\section{LIST OF TERMS}

BHI Bechtel Hanford, Incorporated

CERCLA Comprehensive Environmental Response, Compensation, and Liability Act of 1980

CFR Code of Federal Regulations

DCG derived concentration guide

DOE U.S. Department of Energy

Ecology State of Washington Department of Ecology

EDE effective dose equivalent

EDP Code Electronic Data Processing Code

EP

EPA

external publication

FFTF Fast Flux Test Facility

HEPA high-efficiency particulate air (filter)

LWDF Liquid Waste Disposal Facility

MASF Maintenance and Storage Facility

MEI maximally exposed individual

mrem millirem (unit of dose)

ND not detected

NPDES National Pollutant Discharge Elinination System

PFP Plutonium Finishing Plant

PSD Prevention of Significant Deterioration

PNL Pacific Northwest Laboratory

POTW publicly owned treatment works (City of Richland)

ppm parts per million

PUREX Plutonium-Uranium Extraction

RCRA Resource Conservation and Recovery Act of 1976

REDOX Reduction-Oxidation

RL U.S. Department of Energy, Richland Operations Office

RQ reportable quantity

TEDF Treated Effluent Disposal Facility

TRIGA Test Reactor and Isotope Production, General Atomics

TRU transuranic (waste)

$\mathrm{UO}_{3} \quad$ uranium trioxide

WAC Washington Administrative Code

WESF Waste Encapsulation Storage Facility

WHC Westinghouse Hanford Company 
WHC-EP-0527-5

This page intentionally left blank. 
WHC-EP-0527-5

\section{ENVIRONMENTAL RELEASES FOR \\ CALENDAR YEAR 1995}

\subsection{INTRODUCTION}

The Westinghouse Hanford Company (WHC) and Bechtel Hanford, Incorporated (BHI) are responsible for monitoring radioactive and nonradioactive material released into the environment from DOE facilities at the Hanford Site. Bechtel Hanford, Incorporated has contracted WHC to perform some of its effluent monitoring duties, which includes fulfilling its annual effluent reporting requirements. This report documents the environmental releases from facilities managed by WHC and monitored by BHI during calendar year 1995. The monitored facilities are located in the 100 Areas (reactor areas), 200 Areas (separations areas), 300 and 400 Areas (research and development areas), 1100 Area (warehouses, stores, and offices), and the 600 Area (all remaining areas of the Hanford Site).

\subsection{PURPOSE}

This report fulfills the annual environmental release reporting requirements of U.S. Department of Energy (DOE) Orders 5484.1 and 5400.1 (DOE 1990a and DOE 1990b). This report provides supplemental information to the Hanford Site Environmental Report for Calendar Year 1995 (PNNL, 1996). The Hanford Site Environmental Report provides an update on the environmental status of the entire Hanford Site. The sitewide annual report summarizes the degree of compliance of the Hanford Site with applicable environmental regulations and informs the public about the impact of Hanford operations on the surrounding environment.

Like the Hanford Site Environmental Report, this annual report presents a summary of the environmental releases from facilities managed by WHC and monitored by BHI. In addition to the summary data, this report also includes detailed data on air emissions, liquid effluents, and hazardous substances released to the environment from these facilities, during calendar year 1995 .

\subsection{SCOPE}

This report presents information and data on liquid effluents, air emissions, and nonroutine hazardous substance releases that entered the environment in 1995 from facilities managed by WHC and monitored by BHI. When appropriate, comparisons are made to past releases. 


\subsection{TYPES AND LOCATIONS OF RELEASES}

Radioactive liquid effluents and air emissions are released from facilities in the 100, 200, 300, and 400 Areas. Radioactive liquid effluents are discharged to the soil in the 200 and 300 Areas and to the Columbia River at the $100-\mathrm{N}$ and $100-\mathrm{K}$ Areas.

The major potential sources of nonradioactive air emissions of industrial origin are (1) fossil-fuel combustion emissions from powerhouse facilities in the 200 and 300 Areas, (2) ammonia emissions from liquid radioactive waste tanks and the operation of the 242-A Evaporator, (3) carbon tetrachloride emissions from passively vented wells and the $\mathrm{CCl}_{4}$ Vapor Extraction Project, and (4) nitrogen-oxide $\left(\mathrm{NO}_{\mathrm{x}}\right)$ emissions from the Plutonium-Uranium Extraction (PUREX) Plant . During 1995, the PUREX Plant did not operate and therefore did not emit any $\mathrm{NO}_{\mathrm{x}}$.

Waste water from the powerhouse facilities and water treatment facilities in the 200 Areas is discharged to the soil. In the 300 Areas, filter backwash from the water treatment plant is routed to a pond. Sanitary waste water is discharged to (1) the 100-N sanitary sewage lagoon or septic tanks or drain fields in the 100-B, 100-D, 100-H, and 100-K Areas, (2) several septictank or subsurface disposal systems in the 200 Areas, (3) a septic-tank trench system in the 300 Area, and (4) the sewage treatment plant and lagoon in the 400 Area. Solid waste from offices is buried at the Central Landfill in the 600 Area.

\subsection{ENVIRONMENTAL RELEASE LIMITS AND GUIDELINES}

This section presents environmental release standards. Relevant limits and guidelines for nonradioactive constituents also are included in this section. Guidelines are applicable for nonradioactive constituents when they: (1) affect the release and transport of radioactive constituents, (2) are necessary to meet any issued Federal, State, or local permit, or (3) are necessary to meet any Federal, State, or local regulations or guidelines prescribed by the U.S. Department of Energy, Richland Operations Office (RL).

\subsubsection{Limits for Radioactive Releases}

Concentrations of radionuclides in air emissions and liquid effluents from Hanford facilities are regulated by DOE Order 5400.5, Radiation Protection of the Public and the Environment (DOE 1993a) and Title 40 of the Code of Federal Regulations Part 61, Subpart H (EPA 1989). The effective dose equivalent (EDE) received by any member of the offsite public from all effluents and emissions released during routine operations at the Hanford Site is not to exceed $100 \mathrm{mrem} / \mathrm{yr}$ from continuous exposure throughout a prolonged period (5 years) and $500 \mathrm{mrem} / \mathrm{yr}$ from noncontinuous, occasional exposure. From the air pathway only, the EDE to any member of the public is not to exceed $10 \mathrm{mrem} / \mathrm{yr}$.

The derived concentration guide (DCG) values in DOE Order 5400.5 apply at the location of actual exposure to members of the public. DCG values are used for comparison purposes only. 
$500 \mathrm{mrem} / \mathrm{yr}$ from noncontinuous, occasional exposure. From the air pathway only, the EDE to any member of the public is not to exceed $10 \mathrm{mrem} / \mathrm{yr}$.

The derived concentration guide (DCG) values in DOE Order 5400.5 apply at the location of actual exposure to members of the public. DCG values are used for comparison purposes only.

The Pacific Northwest National Laboratory (PNNL), operated for DOE by Battelle Memorial Institute, issues the annual environmental summary report for the Hanford Site (PNNL 1996) as required by DOE Order 5400.1 . This report assesses the radiological impact to the public resulting from all Hanford Site operations, in accordance with DOE Order 5480.1B (DOE 1993b) and DOE Order 5400.5 (DOE 1993a). The PNNL report uses the release data contained in this report and the Radionuclide Air Emissions Report for the Hanford Site Calendar Year 1995 (DOE-RL 1996) to calculate the offsite radiological dose impact. The PNNL report is used to verify that Hanford Site is in compliance with the standards in DOE Order 5400.5.

\subsubsection{Limits for Nonradioactive Releases}

Nitrogen oxides emitted from the PUREX Plant are regulated in accordance with the limits defined in Prevention of Significant Deterioration Permit No. PSD-X80-14. All other nonradioactive emissions are reported annually to Ecology, as required.

The Resource Conservation and Recovery Act of 1976 (RCRA), the Comprehensive Environmental Response, Compensation, and Liability Act of 1980 (CERCLA), and WAC 173-303 also regulate nonradioactive constituents in air emissions and liquid effluents. Releases and spills of waste materials are reported immediately to appropriate Federal, State, and local agencies when required by CERCLA or WAC 173-303-145. Reporting to the National Response Center is required under CERCLA when the released or spilled material exceeds the reportable quantities listed in 40 CFR 302. Reporting to Ecology is required when a dangerous waste or hazardous substanceis spilled or released into the environment, such that human health or the environment is threatened, regardless of quantity, as defined in WAC 173-303-145. This report summarizes significant releases and spills reported in 1995 from WHC and BHI facilities.

The Westinghouse Hanford Company manages nonradioactive hazardous solid waste in accordance with WAC 173-303. These wastes are kept at a permit-approved interim storage facility before eventual transport to a recycling center or to an approved offsite disposal facility. Nonradioactive, nonhazardous solid waste is handled in accordance with accepted practices for solid-waste management. 


\section{WHC-EP-0527-5}

This page intentionally left blank. 
WHC-EP-0527-5

\subsection{AIR EMISSIONS}

Both radioactive and nonradioactive air emissions to the atmosphere from facilities managed by WHC and monitored by BHI. Release data for each type of emission are discussed separately.

\subsection{RADIONUCLIDE AIR EMISSIONS}

Radionuclide air emissions generally are discharged from stacks and vents. In the 200 Areas, stacks and vents are designated by a number that has a "291" or "296" prefix, depending on their height. Stacks in the 200 Areas that are $61 \mathrm{~m}(200 \mathrm{ft})$ tall are designated by a "291" prefix. All other stacks and vents are designated by the "296" prefix. In the 100,300 , and 400 Areas, stacks and vents usually are identified by facility names.

During 1995, 95 emission points monitored by WHC and BHI. Information on all of these emissions points are included in this report.

\subsubsection{Filtration of Radionuclide Air Emissions}

The following methods are used to remove radionuclides from air emissions:

(1) high-efficiency particulate air (HEPA) filters, (2) sand filters, (3) charcoal absorbers (for iodine removal), (4) water scrubbers, (5) deep-bed fiberglass filters, (6) and fiberglass prefilters. Generally at least one stage, and often several stages, of HEPA filtration is used as the final particulate removal method before the air is discharged to the atmosphere. All in-place HEPA filters are required to have an efficiency of $99.95 \%$ in removing airborne particles larger than $0.3 \mu \mathrm{m}$ in diameter. Filter efficiency is routinely tested. Past release data have shown that radionuclide concentrations in many emissions are below the lower limit of analytical detection.

\subsubsection{Radionuclide Air Emissions}

Release data on radionuclide air emissions from facilities, by area, are presented in Table 2-1. Tables 2-2 and 2-3 present data on the radionuclide air emissions from individual stacks and vents. The data consist of radionuclides detected or sampled for, average concentrations, and total activities.

Point source emissions are reported in this document when the following criteria were met during 1995: (1) emission point required continuous monitoring or periodic confirmatory measurements by 40 CFR 61 Subpart H or WAC 246-247, (2) the emission point normally had radionuclide emissions or potentially had radionuclide emissions. Stacks or emission points not included in this report did not meet the previous criteria or were rendered inoperable (e.g. sealed off) before 1995, as indicated by the previous year's annual report.

Radionuclide air emissions from Hanford Site point sources resulted in an offsite dose to the hypothetical maximally exposed individual (MEI) of $0.0029 \mathrm{mrem}$ EDE (see Figure 2-1). This 
Radionuclide air emissions from Hanford Site point sources resulted in an offsite dose to the hypothetical maximally exposed individual (MEI) of 0.0029 mrem EDE (see Figure 2-1). This dose is well below the $10 \mathrm{mrem} / \mathrm{yr}$ EDE standard imposed by the U.S. Environmental Protection Agency (EPA) on radionuclide air emissions (40 CFR 61 Subpart H).

Figure 2-1

Historical Effective Dose Equivalent Received by the Maximally Exposed Individual Located Offsite, from Airborne Radionuclide Emissions"

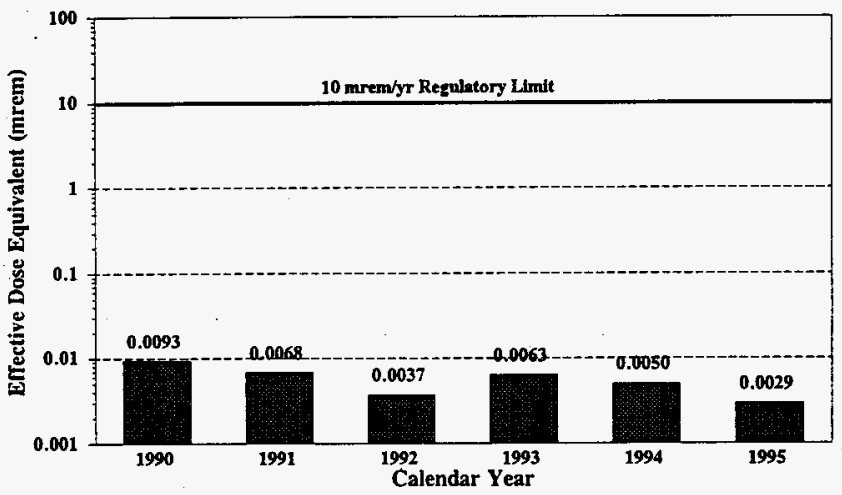

a Includes emissions from PNNL facilities also.

In the 100 Areas, radionuclide emissions at $100-\mathrm{N}$ Area remained below those reported when $\mathrm{N}$ Reactor operated, and the 100-K Area emissions did not change significantly from 1993. In the 200 Areas, radionuclide air emissions accounting for the greatest releases were ${ }^{129} \mathrm{I}$ and ${ }^{241} \mathrm{Am}$ via the PUREX Plant main stack and ${ }^{239.240} \mathrm{Pu}$ from PFP, PUREX Plant, and T Plant. 200 Area emissions have not changed significantly from 1994 emissions. Releases from the 300 and 400 Area facilities did not vary substantially from those reported for 1994 (DOE-RL 1995).

\subsubsection{Nonradioactive Constituents in Radionuclide Air Emissions}

Nitrogen-oxide emissions are released in association with processes at the PUREX and $\mathrm{UO}_{3}$ Plants. The PUREX Plant did not operate in 1995, therefore, no $\mathrm{NO}_{\mathrm{x}}$ emissions were generated or released. Release limits for $\mathrm{NO}_{\mathrm{x}}$ were established by Prevention of Significant Deterioration Permit No. PSD-X80-14, issued by the EPA. 


\subsection{NONRADIOACTIVE AIR EMISSIONS}

In 1995, the significant nonradioactive air emissions were discharged from the following WHC managed facilities: (1) 200 East (284-E) powerhouse, (2) 200 West $284-W$ powerhouse and 284WB oil fired package boiler, and (3) the 300 Area powerhouse, (4) 200 East Area Tank Farms, (5) 200 West Area Tank Farms, (6) 242-A Evaporator, and (7) the 200 West Area $\mathrm{CCl}_{4}$ vapor extraction systems. Data on emissions from these sources are shown in Tables 2-4 and 4-1. Powerhouse stack emissions were calculated using formulas established by the EPA (EPA 1990a).

Fabric-filter collection systems, called baghouses, remove particulate matter and, to a small degree, sulfur oxides $\left(\mathrm{SO}_{x}\right)$, emitted from coal-fired boilers. Baghouses are installed at the coalfired boilers in the 200 Area's Powerhouses 284-E and 284-W. The 284-W Powerhouse's baghouses were shutdown in February 1995. Anytime a baghouse is bypassed (usually because of unavoidable replacement, repair, and maintenance of equipment), RL and the Washington Department of Ecology are notified through a monthly report of excess emissions. 
Table 2-1

\begin{tabular}{|c|c|c|c|c|c|c|}
\hline \multirow[b]{3}{*}{ Radionuclide } & \multicolumn{5}{|c|}{$\begin{array}{l}\text { Release Estimates of } \\
1995 \text { Radionuclide Air Emissions } \\
\text { from WHC and BHI Facilities. }\end{array}$} & \multirow[b]{3}{*}{ Total } \\
\hline & \multicolumn{5}{|c|}{ Release, $\mathrm{Cr}^{\mathbf{Z}}$} & \\
\hline & 100 Areas & $\begin{array}{c}200 \text { East } \\
\text { Area }\end{array}$ & $\begin{array}{c}200 \text { West } \\
\text { Area }\end{array}$ & 300 Area & 400 Area & \\
\hline${ }^{3} \mathrm{H}$ (as HTO) & NM & NM & NM & NM & $2.5 \mathrm{E}-02$ & $2.5 \mathrm{E}-02$ \\
\hline${ }^{60} \mathrm{Co}$ & 9.2 E-06 & NM & NM & ND & NM & $9.2 \mathrm{E}-06$ \\
\hline${ }^{68} \mathrm{Zn}$ & ND & NM & NM & ND & NM & ND \\
\hline${ }^{90} \mathrm{Sr}$ & $6.1 \mathrm{E}-05$ & $6.8 \mathrm{E}-05^{\mathrm{b}}$ & $1.0 \mathrm{E}-04^{\mathrm{b}}$ & $1.1 \mathrm{E}-06^{\mathrm{b}}$ & NM & $2.3 \mathrm{E}-04^{\mathrm{b}}$ \\
\hline${ }^{95} \mathrm{Zr}$ & ND & ND & ND & ND & NM & ND \\
\hline${ }^{106} \mathrm{Ru}$ & $1.1 \mathrm{E}-05$ & 6.7 E-06 & $1.0 \mathrm{E}-08$ & ND & NM & $1.8 \mathrm{E}-05$ \\
\hline${ }^{133} \mathrm{Sn}$ & ND & 8.0 E-07 & $1.4 \mathrm{E}-07$ & ND & NM & $9.4 \mathrm{E}-07$ \\
\hline${ }^{129} \mathrm{Sb}$ & $2.8 \mathrm{E}-06$ & $9.1 \mathrm{E}-06$ & $1.2 \mathrm{E}-07$ & ND & NM & $1.2 \mathrm{E}-05$ \\
\hline${ }^{129} \mathrm{I}$ & NM & 8.9 E-03 & $\mathrm{NM}$ & ND & NM & $8.7 \mathrm{E}-03$ \\
\hline${ }^{131} \mathrm{I}$ & NM & ND & ND & ND & ND & ND \\
\hline${ }^{13.4} \mathrm{Cs}$ & $1.3 \mathrm{E}-06$ & $2.4 \mathrm{E}-08$ & $1.1 \mathrm{E}-07$ & NM & $\mathrm{NM}$ & $1.4 \mathrm{E}-06$ \\
\hline${ }^{137} \mathrm{Cs}$ & $2.5 \mathrm{E}-04$ & $3.8 \mathrm{E}-04$ & $1.9 \mathrm{E}-05$ & ND & $5.9 \mathrm{E}-06^{\mathrm{C}}$ & $6.6 \mathrm{E}-04^{\circ}$ \\
\hline${ }^{152} \mathrm{Eu}$ & ND & $3.7 \mathrm{E}-07$ & $1.6 \mathrm{E}-07$ & ND & NM & $5.2 \mathrm{E}-07$ \\
\hline${ }^{1.5} \mathrm{Eu}$ & $8.3 \mathrm{E}-06$ & $4.6 \mathrm{E}-07$ & $2.6 \mathrm{E}-07$ & ND & NM & $9.1 \mathrm{E}-06$ \\
\hline${ }^{15 s} \mathrm{Eu}$ & $1.5 \mathrm{E}-06$ & $2.2 \mathrm{E}-07$ & $1.2 \mathrm{E}-07$ & $4.3 \mathrm{E}-08$ & NM & $1.8 \mathrm{E}-06$ \\
\hline Uranium. depleted ${ }^{\mathrm{d}}$ & $\mathrm{NM}$ & NM & NM & $2.2 \mathrm{E}-08$ & NM & $2.2 \mathrm{E}-08$ \\
\hline${ }_{238} \mathrm{Pu}$ & $2.3 \mathrm{E}-06$ & $6.9 \mathrm{E}-07$ & $2.6 \mathrm{E}-06$ & NM & NM & $5.6 \mathrm{E}-06$ \\
\hline${ }^{239.240} \mathrm{Pu}$ & $1.5 \mathrm{E}-05$ & $7.9 \mathrm{E}-06^{\mathrm{E}}$ & $1.0 \mathrm{E}-04^{\circ}$ & $3.9 \mathrm{E}-07^{\mathrm{e}}$ & $1.7 \mathrm{E}-06^{\mathrm{e}}$ & $1.3 \mathrm{E}-04^{\circ}$ \\
\hline${ }^{24} \mathrm{Pu}$ & $2.1 \mathrm{E}-04$ & $1.2 \mathrm{E}-04$ & $2.0 \mathrm{E}-04$ & NM & NM & $5.3 \mathrm{E}-04$ \\
\hline${ }^{24 !} \mathrm{Am}$ & 5.7 E-06 & $1.6 \mathrm{E}-05$ & $1.7 \mathrm{E}-05$ & NM & NM & $3.9 \mathrm{E}-05$ \\
\hline
\end{tabular}

Notes:

a $1 \mathrm{Ci}=3.7 \mathrm{E}+10$ Becquerel; $\mathrm{ND}=$ not detected (i.e. either the radionuclide was not detected in any sample during the year, or the average of all the measurements for that given radionuclide or type of radioactivity made during the year was below background levels); $\mathrm{NM}=$ not measured.

b This value includes total beta release data. Total beta results assumed to be ${ }^{90} \mathrm{Sr}$ for dose calculations.

c This value includes total beta release data. Total beta results assumed to be ${ }^{137} \mathrm{Cs}$ for dose calculations from FFTF emissions.

d Determined from total alpha measurements. Assumed to be depleted uranium consisting of $63.478 \mathrm{Ci} \%{ }^{238} \mathrm{U}, 0.821 \mathrm{Ci} \%$ ${ }^{235} \mathrm{U}$, and $35.701 \mathrm{Ci} \%{ }^{234} \mathrm{U}\left(99.797 \mathrm{Wt} \%{ }^{238} \mathrm{U}, 0.200 \mathrm{Wt} \%{ }^{235} \mathrm{U}\right.$, and $\left.0.003 \mathrm{Wt} \%{ }^{234} \mathrm{U}\right)$.

e This value includes total alpha release data. Total alpha results assumed to be ${ }^{239 / 240} \mathrm{Pu}$ for dose calculations. 
WHC-EP-0527-5

Figure 2-2

Historical Airborne Effluent Releases of ${ }^{239240} \mathrm{Pu}$

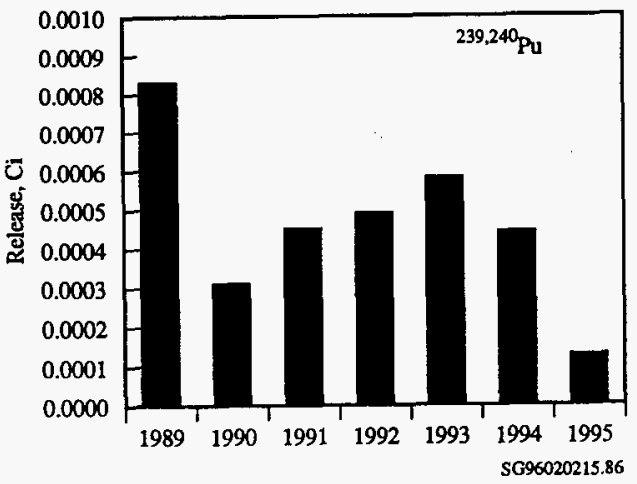

Figure 2-3

Historical Airborne Effluent Releases of ${ }^{129} \mathrm{I}$

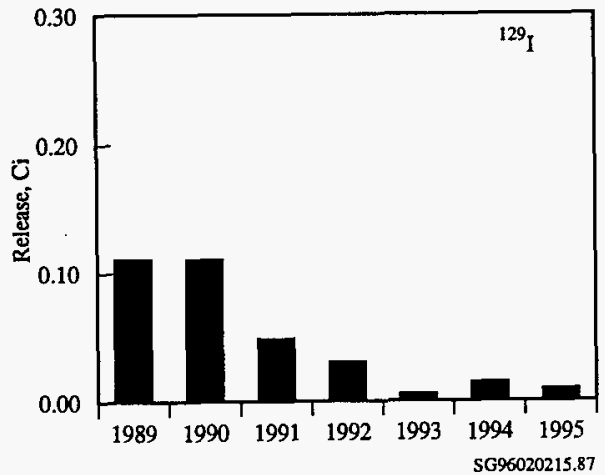


WHC-EP-0527-5

This page intentionally left blank. 
Table 2-2

(6 sheets)

\begin{tabular}{|c|c|c|c|c|c|c|}
\hline \multicolumn{7}{|c|}{$\begin{array}{l}1995 \text { Hanford Site Radionuclide Air Emissions Data } \\
\text { for Major Point Sources from WHC and BHI Facilities. } \\
\text { (major point sources have the potential of }>0.1 \mathrm{mrem} / \mathrm{y} \text { EDE to nearest offsite resident) }\end{array}$} \\
\hline $\begin{array}{l}\text { Source } \mathbf{~ m}^{\mathbf{b}} \\
\text { [Facility/Contractor] } \\
\text { (EDP Code(s)) }\end{array}$ & $\begin{array}{l}\text { Discharge } \\
\text { Height } \\
\text { (m) }\end{array}$ & $\begin{array}{l}\text { Emission } \\
\text { Control }^{\mathfrak{c}} \\
\text { (stages) }^{\text {stag) }}\end{array}$ & $\begin{array}{l}\text { Total } \\
\text { Flow } \\
\left(\mathrm{m}^{3}\right)\end{array}$ & Radionuclide $^{\mathrm{d}}$ & $\begin{array}{c}\text { Average } \\
\text { Concentration } \\
(\mu \mathrm{Ci} / \mathbf{m L})^{\mathrm{e}, f, g}\end{array}$ & $\begin{array}{c}\text { Annual } \\
\text { Emissions } \\
\text { (Ci) })^{e, I, 8}\end{array}$ \\
\hline \multicolumn{7}{|c|}{200 East Area Point Sources } \\
\hline $\begin{array}{l}291-\mathrm{A}-1 \\
\text { [PUREX/WHC] } \\
\text { (A552,A511) }\end{array}$ & 61.0 & HEPA (3) & $1.6 \mathrm{E}+09$ & \begin{tabular}{|l}
${ }^{90} \mathrm{Sr}$ \\
${ }^{106} \mathrm{Ru}$ \\
${ }^{13} \mathrm{Sn}$ \\
${ }^{125} \mathrm{Sb}$ \\
${ }^{129} \mathrm{I}$ \\
${ }^{134} \mathrm{Cs}$ \\
${ }^{137} \mathrm{Cs}$ \\
${ }^{152} \mathrm{Eu}$ \\
${ }^{154} \mathrm{Eu}$ \\
${ }^{155} \mathrm{Eu}$ \\
${ }^{238} \mathrm{Pu}$ \\
$-{ }^{239,240} \mathrm{Pu}$ \\
${ }^{243} \mathrm{Pu}$ \\
- ${ }^{241} \mathrm{Am}$ \\
total aipha \\
total beta
\end{tabular} & $\begin{array}{c}1.4 \mathrm{E}-14 \\
\mathrm{ND} \\
\mathrm{ND} \\
<2.7 \mathrm{E}-15 \\
4.8 \mathrm{E}-12 \\
\mathrm{ND} \\
3.7 \mathrm{E}-14 \\
<1.2 \mathrm{E}-16 \\
<1.1 \mathrm{E}-16 \\
<3.2 \mathrm{E}-17 \\
1.9 \mathrm{E}-16 \\
1.5 \mathrm{E}-15 \\
3.8 \mathrm{E}-14 \\
5.2 \mathrm{E}-15 \\
4.6 \mathrm{E}-15 \\
4.3 \mathrm{E}-14\end{array}$ & $\begin{array}{c}4.2 \mathrm{E}-05 \\
\text { ND } \\
\text { ND } \\
7.4 \mathrm{E}-06 \\
8.6 \mathrm{E}-03 \\
\text { ND } \\
1.0 \mathrm{E}-04 \\
3.5 \mathrm{E}-07 \\
3.2 \mathrm{E}-07 \\
9.4 \mathrm{E}-08 \\
5.7 \mathrm{E}-07 \\
4.5 \mathrm{E}-06 \\
1.1 \mathrm{E}-04 \\
1.6 \mathrm{E}-05 \\
1.4 \mathrm{E}-05 \\
1.3 \mathrm{E}-04\end{array}$ \\
\hline $\begin{array}{l}296-A-1 \\
\text { [PUREX/WHC] } \\
\text { (A540) }\end{array}$ & 20.7 & HEPA (3) & $5.9 \mathrm{E}+07$ & \begin{tabular}{|l}
${ }^{238} \mathrm{Pu}$ \\
${ }^{239}, 240 \mathrm{Pu}$ \\
${ }^{243} \mathrm{Pu}$ \\
- ${ }^{24 !} \mathrm{Am}$ \\
total alpha \\
total beta
\end{tabular} & $\begin{array}{l}1.3 \mathrm{E}-15 \\
2.2 \mathrm{E}-14 \\
1.2 \mathrm{E}-13 \\
6.7 \mathrm{E}-15 \\
1.5 \mathrm{E}-14 \\
1.6 \mathrm{E}-15\end{array}$ & $\begin{array}{ll}1.1 & \mathrm{E}-07 \\
1.8 & \mathrm{E}-06 \\
9.4 & \mathrm{E}-06 \\
5.4 & \mathrm{E}-07 \\
1.2 \mathrm{E}-06 \\
1.3 \mathrm{E}-07\end{array}$ \\
\hline \begin{tabular}{|l}
$291-B-1$ \\
[B Plant/WHC] \\
(B691)
\end{tabular} & 61.0 & HEPA (2) & $5.8 \mathrm{E}+08$ & \begin{tabular}{|l}
${ }^{90} \mathrm{Sr}$ \\
${ }^{106} \mathrm{Ru}$ \\
${ }^{13} \mathrm{Sn}$ \\
${ }^{125} \mathrm{Sb}$ \\
${ }^{134} \mathrm{Cs}$ \\
$\cdot{ }^{137} \mathrm{Cs}$ \\
${ }^{152} \mathrm{Eu}$ \\
${ }^{154} \mathrm{Eu}$ \\
${ }^{155} \mathrm{Eu}$ \\
${ }^{238} \mathrm{Pu}$ \\
- ${ }^{239.240} \mathrm{Pu}$ \\
${ }^{241} \mathrm{Am}$ \\
total alpha \\
total beta
\end{tabular} & $\begin{array}{c}1.1 \mathrm{E}-14 \\
\mathrm{ND} \\
\mathrm{ND} \\
<5.2 \mathrm{E}-17 \\
\mathrm{ND} \\
2.7 \mathrm{E}-13 \\
\mathrm{ND} \\
\mathrm{ND} \\
<8.9 \mathrm{E}-17 \\
1.1 \mathrm{E}-17 \\
1.2 \mathrm{E}-15 \\
8.1 \mathrm{E}-17 \\
1.3 \mathrm{E}-15 \\
1.9 \mathrm{E}-13\end{array}$ & $\begin{array}{c}8.7 \mathrm{E}-06 \\
\mathrm{ND} \\
\mathrm{ND} \\
4.1 \mathrm{E}-08 \\
\text { ND } \\
2.1 \mathrm{E}-04 \\
\text { ND } \\
\mathrm{ND} \\
7.0 \mathrm{E}-08 \\
9.0 \mathrm{E}-09 \\
9.5 \mathrm{E}-07 \\
6.4 \mathrm{E}-08 \\
1.0 \mathrm{E}-06 \\
1.5 \mathrm{E}-04\end{array}$ \\
\hline
\end{tabular}


WHC-EP-0527-5

Table 2-2

(6 sheets)

1995 Hanford Site Radionuclide Air Emissions Data for Major Point Sources from WHC and BHI Facilities. (major point sources have the potential of $>0.1 \mathrm{mrem} / \mathrm{y}$ EDE to nearest offsite resident) ${ }^{\mathrm{a}}$

\begin{tabular}{|c|c|c|c|c|c|c|}
\hline $\begin{array}{c}\text { Source ID } \\
\text { [Facility/Contractor] } \\
\text { (EDP Code(s)) }\end{array}$ & $\begin{array}{l}\text { Discharge } \\
\text { Height } \\
\text { (m) }\end{array}$ & $\begin{array}{l}\text { Emission } \\
\text { Control } \\
\text { (stages) }\end{array}$ & $\begin{array}{l}\text { Total } \\
\text { Flow } \\
\left(\mathrm{m}^{3}\right)\end{array}$ & Radionuclide $^{d}$ & $\begin{array}{c}\text { Average } \\
\text { Concentration } \\
(\mu \mathrm{Ci} / \mathrm{mL})^{e, \mathrm{f}, \mathrm{B}}\end{array}$ & $\begin{array}{c}\text { Annual } \\
\text { Emissions } \\
{\text { (Ci })^{e, f, B}}^{\text {f }}\end{array}$ \\
\hline $\begin{array}{l}296-\mathrm{A}-22 \\
\text { [242-A Evaporator/WHC] } \\
\text { (E643,E002) }\end{array}$ & 18.6 & HEPA (2) & $9.6 \mathrm{E}+06$ & \begin{tabular}{|l}
${ }^{90} \mathrm{Sr}$ \\
${ }^{106} \mathrm{Ru}$ \\
${ }^{113} \mathrm{Sn}$ \\
${ }^{125} \mathrm{Sb}$ \\
${ }^{129} \mathrm{I}$ \\
${ }^{134} \mathrm{Cs}$ \\
${ }^{137} \mathrm{Cs}$ \\
${ }^{152} \mathrm{Eu}$ \\
${ }^{154} \mathrm{Eu}$ \\
${ }^{155} \mathrm{Eu}$ \\
${ }^{238} \mathrm{Pu}$ \\
${ }^{239,240} \mathrm{Pu}$ \\
${ }^{241} \mathrm{Am}$ \\
total alpha \\
total beta
\end{tabular} & $\begin{array}{c}4.5 \text { E-15 } \\
4.4 \text { E-14 } \\
<4.2 \text { E-15 } \\
<6.7 \text { E-15 } \\
3.4 \text { E-13 } \\
5.3 \text { E-17 } \\
4.6 \text { E-17 } \\
\text { ND } \\
\text { ND } \\
<3.3 \text { E-17 } \\
1.4 \text { E-18 } \\
1.4 \text { E-17 } \\
3.3 \text { E-17 } \\
2.8 \text { E-16 } \\
4.0 \text { E-15 }\end{array}$ & $\begin{array}{c}5.4 \text { E-08 } \\
4.8 \mathrm{E}-07 \\
4.7 \mathrm{E}-08 \\
7.4 \mathrm{E}-08 \\
3.7 \mathrm{E}-06 \\
6.4 \mathrm{E}-10 \\
5.5 \mathrm{E}-10 \\
\mathrm{ND} \\
\mathrm{ND} \\
4.0 \mathrm{E}-10 \\
1.7 \mathrm{E}-11 \\
1.8 \mathrm{E}-10 \\
4.0 \mathrm{E}-10 \\
3.4 \mathrm{E}-09 \\
4.9 \mathrm{E}-08\end{array}$ \\
\hline $\begin{array}{l}\text { 296-A-12 } \\
\text { [East Tank Farms/WHC] } \\
\text { (E058) }\end{array}$ & 45.7 & HEPA (2) & $0.0 \mathrm{E}+00$ & - ${ }^{90} \mathrm{Sr}$ & \multicolumn{2}{|c|}{ (did not operate) } \\
\hline $\begin{array}{l}296-\mathrm{A}-17 \\
296-\mathrm{P}-26 \text { (backup) } \\
\text { [East Tank Farms/WHC] } \\
\text { (E059,E026,E027) } \\
\text { (E039,E040,E041) }\end{array}$ & 15.2 & HEPA (2) & $9.2 \mathrm{E}+07$ & \begin{tabular}{|l}
${ }^{90} \mathrm{Sr}$ \\
${ }^{106} \mathrm{Ru}$ \\
${ }^{113} \mathrm{Sn}$ \\
${ }^{125} \mathrm{Sb}$ \\
${ }^{129} \mathrm{I}$ \\
${ }^{134} \mathrm{Cs}$ \\
${ }^{137} \mathrm{Cs}$ \\
${ }^{152} \mathrm{Eu}$ \\
${ }^{154} \mathrm{Eu}$ \\
${ }^{155} \mathrm{Eu}$ \\
${ }^{238} \mathrm{Pu}$ \\
${ }^{239,240} \mathrm{Pu}$ \\
${ }^{241} \mathrm{Am}$ \\
total alpha \\
total beta
\end{tabular} & $\begin{array}{c}5.5 \mathrm{E}-14 \\
5.0 \mathrm{E}-14 \\
<6.6 \mathrm{E}-15 \\
<1.2 \mathrm{E}-14 \\
3.4 \mathrm{E}-12 \\
1.8 \mathrm{E}-16 \\
6.6 \mathrm{E}-13 \\
\mathrm{ND} \\
<9.0 \mathrm{E}-17 \\
<3.2 \mathrm{E}-16 \\
4.7 \mathrm{E}-17 \\
1.5 \mathrm{E}-16 \\
1.2 \mathrm{E}-16 \\
2.2 \mathrm{E}-15 \\
3.7 \mathrm{E}-13\end{array}$ & $\begin{array}{l}5.4 \mathrm{E}-06 \\
4.0 \mathrm{E}-06 \\
5.3 \mathrm{E}-07 \\
9.5 \mathrm{E}-07 \\
2.7 \mathrm{E}-04 \\
1.8 \mathrm{E}-08 \\
6.2 \mathrm{E}-05 \\
\mathrm{ND} \\
8.8 \mathrm{E}-09 \\
3.1 \mathrm{E}-08 \\
4.6 \mathrm{E}-09 \\
1.4 \mathrm{E}-08 \\
1.2 \mathrm{E}-08 \\
2.2 \mathrm{E}-07 \\
3.6 \mathrm{E}-13\end{array}$ \\
\hline
\end{tabular}


Table 2-2

(6 sheets)

\begin{tabular}{|c|c|c|c|c|c|c|}
\hline \multicolumn{7}{|c|}{$\begin{array}{l}1995 \text { Hanford Site Radionuclide Air Emissions Data } \\
\text { for Major Point Sources from WHC and BHI Facilities. } \\
\text { (major point sources have the potential of }>0.1 \mathrm{mrem} / \mathrm{y} \text { EDE to nearest offsite resident) }\end{array}$} \\
\hline $\begin{array}{l}\text { Source } \text { ID }^{\mathrm{b}} \\
\text { [Facility/Contractor] } \\
\text { (EDP Code(s)) }\end{array}$ & $\begin{array}{l}\text { Discharge } \\
\text { Height } \\
\text { (m) }\end{array}$ & $\begin{array}{l}\text { Emission } \\
\text { Control }^{c} \\
\text { (stages) }\end{array}$ & $\begin{array}{l}\text { Total } \\
\text { Flow } \\
\left(\mathbf{m}^{3}\right)\end{array}$ & Radionuclide ${ }^{\mathrm{d}}$ & $\begin{array}{l}\text { Average } \\
\text { Concentration } \\
(\mu \mathrm{Ci} / \mathrm{mL})^{e, f, 8}\end{array}$ & $\begin{array}{l}\text { Annual } \\
\text { Emissions } \\
\text { (Ci) }^{\mathrm{e}, \mathrm{C} ; \mathrm{g}}\end{array}$ \\
\hline $\begin{array}{l}296-\mathrm{A}-25 \\
\text { [East Tank Farms/WHC] } \\
\text { (E080) }\end{array}$ & 3.0 & HEPA (2) & $2.6 \mathrm{E}+06$ & $\begin{array}{l}{ }^{90} \mathrm{Sr} \\
{ }^{106} \mathrm{Ru} \\
{ }^{1 / 3} \mathrm{Sn} \\
{ }^{125} \mathrm{Sb} \\
{ }^{134} \mathrm{Cs} \\
{ }^{137} \mathrm{Cs} \\
{ }^{152} \mathrm{Eu} \\
{ }^{154} \mathrm{Eu} \\
{ }^{155} \mathrm{Eu} \\
{ }^{238} \mathrm{Pu} \\
{ }^{239.240} \mathrm{Pu} \\
{ }^{241} \mathrm{Am} \\
\text { total alpha } \\
\text { total beta }\end{array}$ & $\begin{array}{c}9.9 \text { E-15 } \\
\text { ND } \\
\text { ND } \\
<1.2 \text { E-16 } \\
1.2 \text { E-17 } \\
4.2 \text { E-13 } \\
\text { ND } \\
<4.3 \text { E-17 } \\
\text { ND } \\
1.8 \text { E-17 } \\
5.1 \text { E-17 } \\
1.3 \text { E-16 } \\
1.7 \text { E-15 } \\
3.9 \text { E-13 }\end{array}$ & $\begin{array}{c}3.2 \mathrm{E}-08 \\
\mathrm{ND} \\
\mathrm{ND} \\
4.0 \mathrm{E}-10 \\
4.0 \mathrm{E}-11 \\
1.4 \mathrm{E}-06 \\
\mathrm{ND} \\
1.4 \mathrm{E}-10 \\
\mathrm{ND} \\
5.7 \mathrm{E}-11 \\
1.7 \mathrm{E}-10 \\
4.1 \mathrm{E}-10 \\
5.6 \mathrm{E}-09 \\
1.2 \mathrm{E}-06\end{array}$ \\
\hline $\begin{array}{l}296-\mathrm{A}-40 \\
\text { [East Tank Farms/WHC] } \\
(\mathrm{E} 013, \mathrm{E} 028 . \mathrm{E} 029)\end{array}$ & 4.1 & HEPA (2) & $1.4 \mathrm{E}+07$ & $\begin{array}{l}{ }^{90} \mathrm{Sr} \\
{ }^{106} \mathrm{Ru} \\
{ }^{11} \mathrm{Sn} \\
{ }^{125} \mathrm{Sb} \\
{ }^{129} \mathrm{~J} \\
{ }^{134} \mathrm{Cs} \\
{ }^{137} \mathrm{Cs} \\
{ }^{152} \mathrm{Eu} \\
{ }^{154} \mathrm{Eu} \\
{ }^{155} \mathrm{Eu} \\
{ }^{238} \mathrm{Pu} \\
{ }^{239.240} \mathrm{Pu} \\
{ }^{241} \mathrm{Am} \\
\text { total alpha } \\
\text { total beta }\end{array}$ & $\begin{array}{c}2.4 \text { E-16 } \\
4.9 \text { E-14 } \\
<5.9 \text { E-15 } \\
<1.2 \text { E-14 } \\
9.6 \text { E-16 } \\
2.5 \text { E-17 } \\
1.7 \text { E-15 } \\
<7.7 \text { E-17 } \\
\text { ND } \\
\text { ND } \\
\text { ND } \\
3.3 \text { E-18 } \\
2.8 \text { E-17 } \\
3.7 \text { E-16 } \\
6.9 \text { E-15 }\end{array}$ & $\begin{array}{l}4.3 \mathrm{E}-09 \\
8.1 \mathrm{E}-07 \\
9.8 \mathrm{E}-08 \\
2.0 \mathrm{E}-07 \\
1.6 \mathrm{E}-05 \\
4.5 \mathrm{E}-10 \\
2.7 \mathrm{E}-08 \\
1.4 \mathrm{E}-09 \\
\mathrm{ND} \\
\mathrm{ND} \\
\mathrm{ND} \\
5.8 \mathrm{E}-11 \\
4.9 \mathrm{E}-10 \\
6.6 \mathrm{E}-09 \\
1.2 \mathrm{E}-07\end{array}$ \\
\hline $\begin{array}{l}\text { 296-B-28 } \\
\text { [East Tank Farms/WHC] } \\
\text { (E886) }\end{array}$ & 3.4 & HEPA (2) & $4.2 \mathrm{E}+06$ & $\begin{array}{l}{ }^{90} \mathrm{Sr} \\
{ }^{106} \mathrm{Ru} \\
{ }^{113} \mathrm{Sr} \\
{ }^{125} \mathrm{Sb} \\
{ }^{134} \mathrm{Cs} \\
{ }^{137} \mathrm{Cs} \\
{ }^{152} \mathrm{Eu} \\
{ }^{154} \mathrm{Eu} \\
{ }^{155} \mathrm{Eu} \\
{ }^{238} \mathrm{Pu} \\
{ }^{239,240} \mathrm{Pu} \\
{ }^{241} \mathrm{Am} \\
\text { total alpha } \\
\text { total beta }\end{array}$ & $\begin{array}{c}2.1 \mathrm{E}-15 \\
\mathrm{ND} \\
<7.3 \mathrm{E}-17 \\
<8.3 \mathrm{E}-17 \\
\mathrm{ND} \\
4.6 \mathrm{E}-16 \\
<5.4 \mathrm{E}-17 \\
\mathrm{ND} \\
<7.3 \mathrm{E}-17 \\
\mathrm{ND} \\
1.2 \mathrm{E}-17 \\
2.2 \mathrm{E}-17 \\
3.1 \mathrm{E}-16 \\
2.9 \mathrm{E}-15\end{array}$ & $\begin{array}{c}1.2 \mathrm{E}-08 \\
\mathrm{ND} \\
4.2 \mathrm{E}-10 \\
4.8 \mathrm{E}-10 \\
\mathrm{ND} \\
2.7 \mathrm{E}-09 \\
3.1 \mathrm{E}-10 \\
\mathrm{ND} \\
4.2 \mathrm{E}-10 \\
\mathrm{ND} \\
7.1 \mathrm{E}-11 \\
1.3 \mathrm{E}-10 \\
1.8 \mathrm{E}-09 \\
1.7 \mathrm{E}-08\end{array}$ \\
\hline
\end{tabular}


Table 2-2

(6 sheets)

\begin{tabular}{|c|c|c|c|c|c|c|}
\hline \multicolumn{7}{|c|}{$\begin{array}{l}1995 \text { Hanford Site Radionuclide Air Emissions Data } \\
\text { for Major Point Sources from WHC and BHI Facilities. } \\
\text { (major point sources have the potential of }>0.1 \mathrm{mrem} / \mathrm{y} \text { EDE to nearest offsite resident) }\end{array}$} \\
\hline $\begin{array}{l}\text { Source } \text { ID }^{\mathbf{b}} \\
\text { [Facility/Contractor] } \\
\text { (EDP Code(s)) }\end{array}$ & $\begin{array}{c}\text { Discharge } \\
\text { Height } \\
\text { (m) }\end{array}$ & $\begin{array}{l}\text { Emission } \\
\text { Control }^{\varepsilon} \\
\text { (stages) }\end{array}$ & $\begin{array}{l}\text { Total } \\
\text { Flow } \\
\left(\mathbf{m}^{3}\right)\end{array}$ & Radionuclide & $\begin{array}{c}\text { Average } \\
\text { Concentration } \\
(\mu \mathrm{Ci} / \mathrm{mL})^{\mathrm{es}, \mathrm{f}, \mathrm{g}}\end{array}$ & $\begin{array}{c}\text { Annual } \\
\text { Emissions } \\
(\mathbf{C i})^{\text {e., }, \text { s }}\end{array}$ \\
\hline $\begin{array}{l}296-\mathrm{C}-5 \\
\text { [East Tank Farms/WHCl } \\
\text { (E069) }\end{array}$ & 14.6 & HEPA (2) & $\begin{array}{l}.5 \mathrm{E}+07 \\
\\
\\
\end{array}$ & \begin{tabular}{|l}
${ }^{10} \mathrm{Sr}$ \\
${ }^{105} \mathrm{Ru}$ \\
${ }^{113} \mathrm{Sn}$ \\
${ }^{325} \mathrm{Sb}$ \\
${ }^{134} \mathrm{Cs}$ \\
${ }^{137} \mathrm{Cs}$ \\
${ }^{152} \mathrm{Eu}$ \\
${ }^{154} \mathrm{Eu}$ \\
${ }^{155} \mathrm{Eu}$ \\
${ }^{238} \mathrm{Pu}$ \\
${ }^{239,240} \mathrm{Pu}$ \\
${ }^{241} \mathrm{Am}$ \\
total alpha \\
total beta
\end{tabular} & $\begin{array}{c}.0 \mathrm{E}-15 \\
\text { ND } \\
<1.9 \mathrm{E}-17 \\
<7.1 \mathrm{E}-17 \\
4.5 \mathrm{E}-17 \\
3.1 \mathrm{E}-15 \\
\text { ND } \\
<3.9 \mathrm{E}-19 \\
<3.8 \mathrm{E}-17 \\
5.2 \mathrm{E}-18 \\
4.1 \mathrm{E}-17 \\
2.4 \mathrm{E}-17 \\
2.8 \mathrm{E}-16 \\
1.3 \mathrm{E}-14\end{array}$ & $\begin{array}{cc}2.7 & \mathrm{E}-07 \\
\text { ND } \\
1.7 & \mathrm{E}-09 \\
6.4 & \mathrm{E}-09 \\
4.1 & \mathrm{E}-09 \\
2.8 & \mathrm{E}-07 \\
\mathrm{ND} \\
3.5 & \mathrm{E}-11 \\
3.4 & \mathrm{E}-09 \\
4.6 & \mathrm{E}-10 \\
3.7 & \mathrm{E}-09 \\
2.2 & \mathrm{E}-09 \\
2.5 & \mathrm{E}-08 \\
1.1 & \mathrm{E}-06\end{array}$ \\
\hline $\begin{array}{l}\text { 296-P-16 } \\
\text { [East Tank Farms/WHC] } \\
\text { (E068) }\end{array}$ & 4.6 & HEPA (2) & $6.0 \mathrm{E}+07$ & \begin{tabular}{|l}
${ }^{90} \mathrm{Sr}$ \\
${ }^{106} \mathrm{Ru}$ \\
${ }^{113} \mathrm{Sn}$ \\
${ }^{125} \mathrm{Sb}$ \\
${ }^{134} \mathrm{Cs}$ \\
${ }^{137} \mathrm{Cs}$ \\
${ }^{152} \mathrm{Eu}$ \\
${ }^{154} \mathrm{Eu}$ \\
${ }^{155} \mathrm{Eu}$ \\
${ }^{153} \mathrm{Pu}$ \\
.${ }^{239} \cdot 240 \mathrm{Pu}$ \\
${ }^{244} \mathrm{Am}$ \\
total alpha \\
total beta
\end{tabular} & $\begin{array}{c}5.1 \mathrm{E}-15 \\
\text { ND } \\
\text { ND } \\
<4.5 \mathrm{E}-17 \\
\mathrm{ND} \\
1.7 \mathrm{E}-14 \\
<2.1 \mathrm{E}-17 \\
\text { ND } \\
<5.6 \mathrm{E}-17 \\
1.3 \mathrm{E}-17 \\
2.3 \mathrm{E}-18 \\
8.3 \mathrm{E}-17 \\
2.2 \mathrm{E}-16 \\
2.4 \mathrm{E}-14\end{array}$ & $\begin{array}{c}4.2 \mathrm{E}-07 \\
\text { ND } \\
\text { ND } \\
3.7 \mathrm{E}-09 \\
\text { ND } \\
1.4 \mathrm{E}-06 \\
1.7 \mathrm{E}-09 \\
\text { ND } \\
4.6 \mathrm{E}-09 \\
1.1 \mathrm{E}-09 \\
1.9 \mathrm{E}-10 \\
6.8 \mathrm{E}-09 \\
1.8 \mathrm{E}-08 \\
2.0 \mathrm{E}-06\end{array}$ \\
\hline $\begin{array}{l}296-\mathrm{P}-32 \\
296-\mathrm{P}-33 \\
296-\mathrm{P}-34 \\
\text { [East Tank Farms/WHC] } \\
\text { (E301-E399) }\end{array}$ & 4.6 & HEPA & $1.2 \mathrm{E}+06$ & $\begin{array}{l}{ }^{90} \mathrm{Sr} \\
{ }^{113} \mathrm{Sn} \\
{ }^{125} \mathrm{Sb} \\
{ }^{134} \mathrm{Cs} \\
{ }^{137} \mathrm{Cs} \\
{ }^{152} \mathrm{Eu} \\
{ }^{154} \mathrm{Eu} \\
{ }^{155} \mathrm{Eu} \\
{ }^{238} \mathrm{Pu} \\
{ }^{239} \cdot 240 \mathrm{Pu} \\
{ }^{241} \mathrm{Am} \\
\text { total alpha } \\
\text { total beta }\end{array}$ & $\begin{array}{c}2.5 \mathrm{E}-14 \\
<3.8 \mathrm{E}-17 \\
<1.2 \mathrm{E}-15 \\
9.1 \mathrm{E}-16 \\
1.7 \mathrm{E}-15 \\
<3.2 \mathrm{E}-16 \\
<3.4 \mathrm{E}-16 \\
<1.7 \mathrm{E}-15 \\
\text { ND } \\
3.2 \mathrm{E}-16 \\
6.9 \mathrm{E}-16 \\
1.6 \mathrm{E}-15 \\
5.7 \mathrm{E}-16\end{array}$ & $\begin{array}{cc}3.0 & \mathrm{E}-08 \\
4.6 & \mathrm{E}-11 \\
1.4 & \mathrm{E}-09 \\
1.1 & \mathrm{E}-09 \\
2.0 & \mathrm{E}-09 \\
3.9 & \mathrm{E}-10 \\
4.1 & \mathrm{E}-10 \\
2.1 & \mathrm{E}-09 \\
\text { ND } \\
3.9 & \mathrm{E}-10 \\
8.3 & \mathrm{E}-10 \\
1.9 & \mathrm{E}-09 \\
6.9 & \mathrm{E}-10\end{array}$ \\
\hline
\end{tabular}


Table 2-2

(6 sheets)

1995 Hanford Site Radionuclide Air Emissions Data

for Major Point Sources from WHC and BHI Facilities.

(major point sources have the potential of $>0.1 \mathrm{mrem} / \mathrm{y}$ EDE to nearest offsite resident) ${ }^{\mathrm{a}}$

\begin{tabular}{|c|c|c|c|c|c|c|}
\hline $\begin{array}{c}\text { Source } \mathbf{I D}^{\mathbf{b}} \\
\text { [Facility/Contractor] } \\
\text { (EDP Code(s)) }\end{array}$ & $\begin{array}{l}\text { Discharge } \\
\text { Height } \\
\text { (m) }\end{array}$ & $\begin{array}{l}\text { Emission } \\
\text { Control }^{\mathrm{c}} \\
\text { (stages) }\end{array}$ & $\begin{array}{l}\text { Total } \\
\text { Flow } \\
\left(\mathbf{m}^{3}\right)\end{array}$ & Radionuclide $^{\mathbf{d}}$ & $\begin{array}{c}\text { Average } \\
\text { Concentration } \\
(\mu \mathrm{Cl} / \mathrm{mL})^{e, 1 ; 8}\end{array}$ & $\begin{array}{c}\text { Annual } \\
\text { Emissions } \\
\text { (Ci) }^{\text {e,l,g }}\end{array}$ \\
\hline \multicolumn{7}{|c|}{200 West Area Point Sources } \\
\hline $\begin{array}{l}291-\mathrm{Z}-1 \\
\text { [PFP/WHC] } \\
(\mathrm{Z} 810)\end{array}$ & 61.0 & HEPA (1-3) & $3.7 \mathrm{E}+09$ & $\begin{array}{l}{ }^{238} \mathrm{Pu} \\
{ }^{239,240} \mathrm{Pu} \\
{ }^{241} \mathrm{Pu} \\
{ }^{24} \mathrm{Am} \\
\text { total alpha } \\
\text { total beta }\end{array}$ & 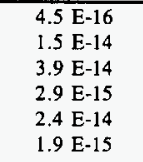 & 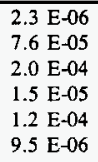 \\
\hline $\begin{array}{l}296-S-15 \\
\text { [West Tank Farms/WHC] } \\
\text { (W111) }\end{array}$ & 4.6 & HEPA (2) & $8.7 \mathrm{E}+07$ & $\begin{array}{l}{ }^{90} \mathrm{Sr} \\
{ }^{106} \mathrm{Ru} \\
{ }^{113} \mathrm{Sn} \\
{ }^{125} \mathrm{Sb} \\
{ }^{134} \mathrm{Cs} \\
{ }^{137} \mathrm{Cs} \\
{ }^{152} \mathrm{Eu} \\
{ }^{154} \mathrm{Eu} \\
{ }^{155} \mathrm{Eu} \\
{ }^{238} \mathrm{Pu} \\
{ }^{239}, 240 \mathrm{Pu} \\
{ }^{241} \mathrm{Am} \\
\text { total alpha } \\
\text { total beta }\end{array}$ & $\begin{array}{c}6.4 \text { E-16 } \\
\text { ND } \\
<2.0 \text { E-17 } \\
\text { ND } \\
1.6 \text { E-17 } \\
1.1 \text { E-15 } \\
<2.6 \text { E-17 } \\
<5.3 \text { E-17 } \\
<6.3 \text { E-17 } \\
5.3 \text { E-18 } \\
1.8 \text { E-17 } \\
2.6 \text { E-17 } \\
2.7 \text { E-16 } \\
4.0 \text { E-15 }\end{array}$ & $\begin{array}{l}6.6 \mathrm{E}-08 \\
\text { ND } \\
2.1 \mathrm{E}-09 \\
\text { ND } \\
1.7 \mathrm{E}-09 \\
1.1 \mathrm{E}-07 \\
2.7 \mathrm{E}-09 \\
5.4 \mathrm{E}-09 \\
6.5 \mathrm{E}-09 \\
5.4 \mathrm{E}-10 \\
1.8 \mathrm{E}-09 \\
2.7 \mathrm{E}-09 \\
2.7 \mathrm{E}-08 \\
4.1 \mathrm{E}-07\end{array}$ \\
\hline $\begin{array}{l}296-\text { S-22 } \\
\text { [West Tank Farms/WHC] } \\
\text { (W880) }\end{array}$ & 3.7 & HEPA (2) & $1.7 \mathrm{E}+06$ & $\begin{array}{l}{ }^{\circ} \mathrm{Sr} \\
{ }^{106} \mathrm{Ru} \\
{ }^{13} \mathrm{Sn} \\
{ }^{125} \mathrm{Sb} \\
{ }^{134} \mathrm{Cs} \\
{ }^{137} \mathrm{Cs} \\
{ }^{152} \mathrm{Eu} \\
{ }^{154} \mathrm{Eu} \\
{ }^{155} \mathrm{Eu} \\
{ }^{238} \mathrm{Pu} \\
{ }^{239.240} \mathrm{Pu} \\
{ }^{241} \mathrm{Am} \\
\text { total alpha } \\
\text { total beta }\end{array}$ & $\begin{array}{c}9.0 \mathrm{E}-15 \\
\mathrm{ND} \\
\mathrm{ND} \\
<1.4 \mathrm{E}-16 \\
\mathrm{ND} \\
1.8 \mathrm{E}-16 \\
\mathrm{ND} \\
\mathrm{ND} \\
<2.2 \mathrm{E}-16 \\
\mathrm{ND} \\
6.0 \mathrm{E}-17 \\
1.8 \mathrm{E}-16 \\
2.6 \mathrm{E}-16 \\
3.6 \mathrm{E}-15\end{array}$ & $\begin{array}{l}2.1 \mathrm{E}-08 \\
\mathrm{ND} \\
\mathrm{ND} \\
3.2 \mathrm{E}-10 \\
\mathrm{ND} \\
4.2 \mathrm{E}-10 \\
\mathrm{ND} \\
\mathrm{ND} \\
5.0 \mathrm{E}-10 \\
\mathrm{ND} \\
1.4 \mathrm{E}-10 \\
4.1 \mathrm{E}-10 \\
6.1 \mathrm{E}-10 \\
8.4 \mathrm{E}-09\end{array}$ \\
\hline
\end{tabular}


Table 2-2

(6 sheets)

\section{Hanford Site Radionuclide Air Emissions Data for Major Point Sources from WHC and BHI Facilities.} (major point sources have the potential of $>0.1 \mathrm{mrem} / \mathrm{y}$ EDE to nearest offsite resident) ${ }^{*}$

\begin{tabular}{|c|c|c|c|c|c|c|}
\hline $\begin{array}{c}\text { Source ID } \\
\text { [Facility/Contractor] } \\
\text { (EDP Code(s)) }\end{array}$ & $\begin{array}{c}\text { Discharge } \\
\text { Height } \\
\text { (m) }\end{array}$ & $\begin{array}{l}\text { Emission } \\
\text { Control } \\
\text { (stages) }\end{array}$ & $\begin{array}{l}\text { Total } \\
\text { Flow } \\
\left(\mathbf{m}^{3}\right)\end{array}$ & Radionuclide ${ }^{d}$ & $\begin{array}{c}\text { Average } \\
\text { Concentration } \\
(\mu \mathrm{Ci} / \mathbf{m L})^{e, f ; \beta}\end{array}$ & $\begin{array}{c}\text { Annual } \\
\text { Emissions } \\
(\mathrm{Ci})^{e, f, 8}\end{array}$ \\
\hline $\begin{array}{l}296-\mathrm{T}-18 \\
\text { [West Tank Farms] } \\
\text { (W882) }\end{array}$ & 3.7 & HEPA (2) & $5.4 \mathrm{E}+06$ & $\begin{array}{l}{ }^{90} \mathrm{Sr} \\
{ }^{100} \mathrm{Ru} \\
{ }^{113} \mathrm{Sn} \\
{ }^{125} \mathrm{Sb} \\
{ }^{134} \mathrm{Cs} \\
{ }^{137} \mathrm{Cs} \\
{ }^{152} \mathrm{Eu} \\
{ }^{154} \mathrm{Eu} \\
{ }^{155} \mathrm{Eu} \\
{ }^{238} \mathrm{Pu} \\
{ }^{239.240} \mathrm{Pu} \\
{ }^{241} \mathrm{Am} \\
\text { total alpha } \\
\text { total beta }\end{array}$ & $\begin{array}{c}1.3 \mathrm{E}-17 \\
\text { ND } \\
\text { ND } \\
\text { ND } \\
3.0 \text { E-17 } \\
3.4 \text { E-15 } \\
\text { ND } \\
\text { ND } \\
<7.9 \text { E-17 } \\
2.7 \text { E-18 } \\
1.3 \text { E-16 } \\
7.4 \text { E-17 } \\
2.3 \text { E-16 } \\
3.3 \text { E-15 }\end{array}$ & $\begin{array}{l}1.0 \mathrm{E}-10 \\
\text { ND } \\
\text { ND } \\
\text { ND } \\
2.3 \text { E-10 } \\
2.6 \text { E-10 } \\
\text { ND } \\
\text { ND } \\
6.2 \text { E-10 } \\
2.1 \text { E-11 } \\
1.0 \text { E-09 } \\
5.8 \text { E-10 } \\
1.8 \text { E-09 } \\
2.5 \text { E-08 }\end{array}$ \\
\hline \multicolumn{7}{|c|}{300 Area Point Sources } \\
\hline $\begin{array}{l}\text { 340-NT-EX } \\
\text { [340 Waste } \\
\text { Handling/WHC] } \\
\text { (F002,F007) }\end{array}$ & 5.5 & $\begin{array}{l}\text { HEPA (2), } \\
\text { charcoal }\end{array}$ & $2.9 E+07$ & $\begin{array}{l}{ }^{90} \mathrm{Sr} \\
{ }^{131} \mathrm{I} \\
{ }^{137} \mathrm{Cs} \\
{ }^{239.240} \mathrm{Pu} \\
\text { total alpha } \\
\text { total beta }\end{array}$ & $\begin{array}{c}\text { ND } \\
\text { ND } \\
\text { ND } \\
\text { ND } \\
1.7 \text { E-15 } \\
3.2 \text { E-15 }\end{array}$ & $\begin{array}{c}\text { ND } \\
\text { ND } \\
\text { ND } \\
\text { ND } \\
6.7 \text { E-08 } \\
1.3 \text { E-07 }\end{array}$ \\
\hline
\end{tabular}

Notes:

a Determining the state of National Emission Standards for Hazardous Air Pollutants (NESHAP) Subpart H compliance for each point source involved using nearest offsite residences, which differed from the MEI.

b ID = Identification. i.e., the alpha-numeric designator for the respective point source; WHC $=$ Westinghouse Hanford Company; BHI = Bechtel Hanford, lnc

c Efficiencies are: $\geq 99.95 \%$ for HEPA; $\geq 95 \%$ for charcoal; $\geq 99.8 \%$ for sand filter; $0 \%$ for no emission control.

d Bullets. "*", identify specific radjonuclide sampling and analysis required by 40 CFR 61 Subpart H.

e $1 \mu \mathrm{Ci} / \mathrm{mL}=3.7 \mathrm{E}+10 \mathrm{bq} / \mathrm{m}^{3} ; \quad 1$ Curie $=3.7 \mathrm{E}+10$ becquerel; $\mathrm{ND}=$ not detected (i.e. the average of all the measurements for that given radionuclide or type of radioactivity made during the year was below background levels).

f Note that the average concentrations do not have sampling system correction factors applied to them. Sampling system correction factors have been applied to the annual emissions estimates.

$\mathrm{g}<=$ less than detectable; Note that all 200 East and 200 West results for gamma emitters ${ }^{113} \mathrm{Sn},{ }^{125} \mathrm{Sb}$, ${ }^{152} \mathrm{Eu}$, ${ }^{154} \mathrm{Eu}$, and ${ }^{155} \mathrm{Eu}$ may have been false positives, since the gamma energy analyses did not identify any energy peaks for these isotopes. These results have been reported, causing emissions estimates to be conservatively higher than they really were. This problem will be corrected, such that false positives are not reported and that emissions estimates are more accurate. 
Table 2-3

(10 sheets)

\begin{tabular}{|c|c|c|c|c|c|c|}
\hline \multicolumn{7}{|c|}{$\begin{array}{l}1995 \text { Hanford Site Radionuclide Air Emissions Data } \\
\text { for Minor Point Sources from WHC and BHI Facilities. } \\
\text { (minor point sources have the potential of }<0.1 \mathrm{mrem} / \mathrm{y} \text { EDE to nearest offsite resident) }\end{array}$} \\
\hline $\begin{array}{l}\text { Source ID } \\
\text { [Facility/Contractor] } \\
\text { (EDP Code(s)) }\end{array}$ & $\begin{array}{l}\text { Discharge } \\
\text { Height } \\
\text { (m) }\end{array}$ & $\begin{array}{l}\text { Emission } \\
\text { Control }^{\mathrm{b}}\end{array}$ & $\begin{array}{l}\text { Total } \\
\text { Flow } \\
\left(\mathbf{m}^{3}\right)\end{array}$ & Radionuclide & $\begin{array}{l}\text { Average } \\
\text { Concentration } \\
(\mu \mathrm{Ci} / \mathrm{mL})^{\mathrm{c}, \mathrm{d}, \mathrm{e}}\end{array}$ & $\begin{array}{l}\text { Annual } \\
\text { Emissions } \\
(\mathrm{Ci})^{\mathrm{c}, \mathrm{d}, \mathrm{e}}\end{array}$ \\
\hline \multicolumn{7}{|c|}{100 Area Point Sources } \\
\hline $\begin{array}{l}116-\mathrm{N} \\
\text { [100 N Area/BHI] } \\
(\mathrm{Y} 211, \mathrm{Y} 212, \mathrm{Y} 213)\end{array}$ & 61.3 & $\begin{array}{l}\text { HEPA. } \\
\text { charcoal }\end{array}$ & $1.6 \mathrm{E}+09$ & $\begin{array}{l}{ }^{60} \mathrm{Co} \\
{ }^{90} \mathrm{Sr} \\
{ }^{130} \mathrm{Cs} \\
{ }^{238} \mathrm{Pu} \\
{ }^{239 / 240} \mathrm{Pu} \\
{ }^{241} \mathrm{Am} \\
\text { total alpha } \\
\text { total beta }\end{array}$ & $\begin{array}{c}2.9 \mathrm{E}-15 \\
\text { ND } \\
\text { ND } \\
\text { ND } \\
2.6 \mathrm{E}-17 \\
1.9 \mathrm{E}-17 \\
2.4 \mathrm{E}-16 \\
4.2 \mathrm{E}-15\end{array}$ & $\begin{array}{c}6.6 \mathrm{E}-06 \\
\text { ND } \\
\text { ND } \\
\text { ND } \\
5.8 \mathrm{E}-08 \\
4.2 \mathrm{E}-08 \\
5.3 \\
\mathrm{E}-07 \\
9.5 \mathrm{E}-06\end{array}$ \\
\hline $\begin{array}{l}105-\mathrm{N} 14 \mathrm{ft} \\
{[100 \mathrm{~N} \mathrm{Area} / \mathrm{BHI}]} \\
(\mathrm{Y} 259 . \mathrm{Y} 260)\end{array}$ & 8.5 & HEPA & $9.5 \mathrm{E}+07$ & $\begin{array}{l}{ }^{60} \mathrm{Co} \\
{ }^{90} \mathrm{Sr} \\
{ }^{125} \mathrm{Sb} \\
{ }^{238} \mathrm{Pu} \\
{ }^{239}{ }^{240} \mathrm{Pu} \\
{ }^{241} \mathrm{Am} \\
\text { total alpha } \\
\text { total beta }\end{array}$ & $\begin{array}{c}\text { ND } \\
3.4 \text { E-15 } \\
2.1 \text { E-15 } \\
\text { ND } \\
1.9 \text { E-17 } \\
1.2 \text { E-16 } \\
4.5 \text { E-17 } \\
6.8 \text { E-16 }\end{array}$ & $\begin{array}{c}\text { ND } \\
4.4 \text { E-07 } \\
2.7 \text { E-07 } \\
\text { ND } \\
2.5 \text { E-09 } \\
1.6 \text { E-08 } \\
5.8 \text { E-09 } \\
8.8 \text { E-16 }\end{array}$ \\
\hline $\begin{array}{l}107-\mathrm{N} \\
{[100 \mathrm{~N} \text { Area/BHI] }} \\
(\mathrm{Y} 265, \mathrm{Y} 266)\end{array}$ & 12.0 & HEPA & $1.1 \mathrm{E}+08$ & $\begin{array}{l}{ }^{60} \mathrm{Co} \\
{ }^{90} \mathrm{Sr} \\
{ }^{55} \mathrm{Eu} \\
{ }^{238} \mathrm{Pu} \\
{ }^{239 / 240} \mathrm{Pu} \\
{ }^{241} \mathrm{Am} \\
\text { total alpha } \\
\text { total beta }\end{array}$ & $\begin{array}{c}6.9 \text { E-16 } \\
9.8 \text { E-16 } \\
1.5 \text { E-15 } \\
\text { ND } \\
1.6 \text { E-17 } \\
2.4 \text { E-17 } \\
2.1 \text { E-17 } \\
6.4 \text { E-16 }\end{array}$ & $\begin{array}{c}1.0 \mathrm{E}-07 \\
1.5 \mathrm{E}-07 \\
2.0 \mathrm{E}-07 \\
\text { ND } \\
2.3 \mathrm{E}-09 \\
3.6 \mathrm{E}-09 \\
3.1 \mathrm{E}-09 \\
9.5 \mathrm{E}-08\end{array}$ \\
\hline $\begin{array}{l}109-\mathrm{N}-\mathrm{Z}-1 \\
{[100 \mathrm{~N} \text { Area/BHI }]} \\
(\mathrm{Y} 221, \mathrm{Y} 222, \mathrm{Y} 223)\end{array}$ & 13.7 & none & $0.0 \mathrm{E}+00$ & \multicolumn{3}{|c|}{ (did not operate) } \\
\hline $\begin{array}{l}109-\mathrm{N}-\mathrm{C}-6 \\
{[100 \mathrm{~N} \text { Area/BHI] }} \\
(\mathrm{Y} 231, \mathrm{Y} 232, \mathrm{Y} 233)\end{array}$ & 13.7 & none & $0.0 \mathrm{E}+00$ & \multicolumn{3}{|c|}{ (did not operate) } \\
\hline
\end{tabular}


Table 2-3

(10 sheets)

\begin{tabular}{|c|c|c|c|c|c|c|}
\hline \multicolumn{7}{|c|}{$\begin{array}{l}1995 \text { Hanford Site Radionuclide Air Emissions Data } \\
\text { for Minor Point Sources from WHC and BHI Facilities. } \\
\text { (minor point sources have the potential of }<0.1 \mathrm{mrem} / \mathrm{y} \text { EDE to nearest offsite resident) }\end{array}$} \\
\hline $\begin{array}{l}\text { Source ID" } \\
\text { [Facility/Contractor] } \\
\text { (EDP Code(s)) }\end{array}$ & $\begin{array}{l}\text { Discharge } \\
\text { Height } \\
\text { (m) }\end{array}$ & $\begin{array}{l}\text { Emission } \\
\text { Control }^{b}\end{array}$ & $\begin{array}{l}\text { Total } \\
\text { Flow } \\
\left(\mathrm{m}^{3}\right)\end{array}$ & Radionuclide & $\begin{array}{l}\text { Average } \\
\text { Concentration } \\
(\mu \mathrm{Ci} / \mathrm{mL})^{\mathrm{c}, \mathrm{d}, \mathrm{e}}\end{array}$ & $\begin{array}{l}\text { Annual } \\
\text { Emissions } \\
(C i)^{c, d, e}\end{array}$ \\
\hline $\begin{array}{l}\text { 105-KE Basin } \\
{[100 \mathrm{~K} \text { Area/WHC }]} \\
\text { (Y245-Y248) }\end{array}$ & 12.8 & none & $5.3 \mathrm{E}+08$ & $\begin{array}{l}{ }^{60} \mathrm{Co} \\
{ }^{90} \mathrm{Sr} \\
{ }^{106} \mathrm{Ru} \\
{ }^{125} \mathrm{Sb} \\
{ }^{134} \mathrm{Cs} \\
{ }^{137} \mathrm{Cs} \\
{ }^{154} \mathrm{Eu} \\
{ }^{155} \mathrm{Eu} \\
{ }^{238} \mathrm{Pu} \\
{ }^{239 / 240} \mathrm{Pu} \\
{ }^{241} \mathrm{Pu} \\
{ }^{241} \mathrm{Am} \\
{ }^{10 t a l} \text { alpha } \\
\text { total beta }\end{array}$ & $\begin{array}{ll}3.6 & \text { E-15 } \\
1.1 & \text { E-13 } \\
2.1 & \text { E-14 } \\
4.7 & \text { E-15 } \\
8.6 & \text { E-16 } \\
4.5 & \text { E-13 } \\
1.1 & \text { E-14 } \\
2.2 & \text { E-15 } \\
4.3 & \text { E-15 } \\
2.8 & \text { E-14 } \\
3.9 & \text { E-13 } \\
1.0 & \text { E-14 } \\
5.1 & \text { E-14 } \\
5.6 & \text { E-13 }\end{array}$ & $\begin{array}{ll}1.9 & \mathrm{E}-06 \\
5.9 & \mathrm{E}-05 \\
1.1 & \mathrm{E}-05 \\
2.5 & \mathrm{E}-06 \\
4.6 & \mathrm{E}-07 \\
2.4 & \mathrm{E}-04 \\
5.8 & \mathrm{E}-06 \\
1.2 & \mathrm{E}-06 \\
2.3 & \mathrm{E}-06 \\
1.5 & \mathrm{E}-05 \\
2.1 & \mathrm{E}-04 \\
5.6 & \mathrm{E}-06 \\
2.7 & \mathrm{E}-05 \\
3.0 & \mathrm{E}-04\end{array}$ \\
\hline $\begin{array}{l}\text { 105-KW Basin } \\
{[100 \mathrm{~K} \text { Area/WHC }]} \\
(\mathrm{Y} 234-\mathrm{Y} 236)\end{array}$ & 12.8 & none & $5.0 \mathrm{E}+08$ & $\begin{array}{l}{ }^{60} \mathrm{Co} \\
{ }^{90} \mathrm{Sr} \\
{ }^{134} \mathrm{Cs} \\
{ }^{137} \mathrm{Cs} \\
{ }^{154} \mathrm{Eu} \\
{ }^{155} \mathrm{Eu} \\
{ }^{238} \mathrm{Pu} \\
{ }^{239 / 240} \mathrm{Pu} \\
{ }^{241} \mathrm{Pu} \\
{ }^{241} \mathrm{Am} \\
\text { total alpha } \\
\text { total beta }\end{array}$ & $\begin{array}{ll}1.1 & \text { E-15 } \\
2.0 & \text { E-15 } \\
1.7 & \text { E-15 } \\
2.6 & \text { E-14 } \\
3.8 & \text { E-15 } \\
1.5 & \text { E-17 } \\
2.4 & \text { E-17 } \\
1.4 & \text { E-15 } \\
2.8 & \text { E-16 } \\
1.2 & \text { E-16 } \\
3.4 & \text { E-15 } \\
4.4 & \text { E-14 }\end{array}$ & 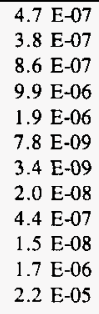 \\
\hline $\begin{array}{l}\text { 1706-KER }-27 \mathrm{ft} \\
{[100 \mathrm{~K} \text { Area/WHC] }} \\
(\mathrm{Y} 244)\end{array}$ & 0.9 & HEPA & $3.7 \mathrm{E}+07$ & $\begin{array}{l}{ }^{60} \mathrm{Co} \\
{ }^{90} \mathrm{Sr} \\
{ }^{134} \mathrm{Cs} \\
{ }^{137} \mathrm{Cs} \\
{ }^{238} \mathrm{Pu} \\
{ }^{239 / 240} \mathrm{Pu} \\
{ }^{241} \mathrm{Am} \\
\text { total alpha } \\
\text { total beta }\end{array}$ & $\begin{array}{c}\text { ND } \\
1.6 \mathrm{E}-16 \\
3.3 \mathrm{E}-16 \\
\text { ND } \\
2.5 \mathrm{E}-18 \\
1.8 \mathrm{E}-17 \\
1.9 \mathrm{E}-17 \\
2.6 \mathrm{E}-16 \\
1.9 \mathrm{E}-15\end{array}$ & $\begin{array}{c}\text { ND } \\
6.0 \mathrm{E}-09 \\
1.2 \mathrm{E}-08 \\
\mathrm{ND} \\
9.1 \mathrm{E}-11 \\
6.5 \mathrm{E}-10 \\
6.9 \mathrm{E}-10 \\
9.5 \mathrm{E}-09 \\
7.0 \mathrm{E}-08\end{array}$ \\
\hline $\begin{array}{l}1706-\mathrm{KE} \\
{[100 \mathrm{~K} \text { Area/WHC] }} \\
(\mathrm{Y} 243)\end{array}$ & 7.6 & HEPA & $1.8 \mathrm{E}+08$ & $\begin{array}{l}{ }^{60} \mathrm{Co} \\
{ }^{90} \mathrm{Sr} \\
{ }^{137} \mathrm{Cs} \\
{ }^{154} \mathrm{Eu} \\
{ }^{155} \mathrm{Eu} \\
{ }^{239: 240} \mathrm{Pu} \\
{ }^{241} \mathrm{Am} \\
\text { total alpha } \\
\text { total beta }\end{array}$ & $\begin{array}{c}1.8 \mathrm{E}-16 \\
\text { ND } \\
\text { ND } \\
3.4 \mathrm{E}-15 \\
7.3 \mathrm{E}-16 \\
2.0 \mathrm{E}-17 \\
5.0 \mathrm{E}-17 \\
1.3 \mathrm{E}-16 \\
7.3 \mathrm{E}-16\end{array}$ & $\begin{array}{c}3.3 \mathrm{E}-08 \\
\mathrm{ND} \\
\mathrm{ND} \\
6.0 \mathrm{E}-07 \\
1.3 \mathrm{E}-07 \\
3.6 \mathrm{E}-09 \\
9.0 \mathrm{E}-09 \\
2.4 \mathrm{E}-08 \\
1.3 \mathrm{E}-07\end{array}$ \\
\hline
\end{tabular}


Table 2-3

(10 sheets)

\begin{tabular}{|c|c|c|c|c|c|c|}
\hline \multicolumn{7}{|c|}{$\begin{array}{l}1995 \text { Hanford Site Radionuclide Air Emissions Data } \\
\text { for Minor Point Sources from WHC and BHI Facilities. } \\
\text { (minor point sources have the potential of }<0.1 \mathrm{mrem} / \mathrm{y} \text { EDE to nearest offsite resident) }\end{array}$} \\
\hline $\begin{array}{l}\text { Source } \mathrm{ID}^{2} \\
\text { [Facility/Contractor] } \\
\text { (EDP Code(s)) }\end{array}$ & $\begin{array}{c}\text { Discharge } \\
\text { Height } \\
\text { (m) }\end{array}$ & $\begin{array}{l}\text { Emission } \\
\text { Control }^{\mathrm{b}}\end{array}$ & $\begin{array}{c}\text { Total } \\
\text { Flow } \\
\left(\mathbf{m}^{3}\right)\end{array}$ & Radionuclide & $\begin{array}{c}\text { Average } \\
\text { Concentration } \\
(\mu \mathrm{Ci} / \mathrm{mL})^{\mathrm{c}, \boldsymbol{d}, \mathrm{e}}\end{array}$ & $\begin{array}{c}\text { Annual } \\
\text { Emissions } \\
(\mathrm{Ci})^{\mathrm{e}, \mathrm{d}, \mathrm{e}}\end{array}$ \\
\hline \multicolumn{7}{|c|}{200 East Area Point Sources } \\
\hline $\begin{array}{l}296-\mathrm{A}-2 \\
\text { [PUREX/WHC] } \\
\text { (A542) }\end{array}$ & 20.7 & HEPA & $3.3 \mathrm{E}+07$ & $\begin{array}{l}\text { total alpha } \\
\text { total beta }\end{array}$ & $\begin{array}{l}7.3 \mathrm{E}-17 \\
1.3 \mathrm{E}-16\end{array}$ & $\begin{array}{l}3.3 \mathrm{E}-09 \\
5.8 \mathrm{E}-09\end{array}$ \\
\hline $\begin{array}{l}296-A-3 \\
\text { [PUREX/WHC] } \\
\text { (A543) }\end{array}$ & 24.1 & HEPA & $4.4 \mathrm{E}+07$ & $\begin{array}{l}\text { total alpha } \\
\text { total beta }\end{array}$ & $\begin{array}{l}8.6 \mathrm{E}-17 \\
1.8 \mathrm{E}-15\end{array}$ & $\begin{array}{l}5.2 \mathrm{E}-09 \\
1.1 \mathrm{E}-07\end{array}$ \\
\hline $\begin{array}{l}296-\mathrm{A}-5 \mathrm{~A} \\
296-\mathrm{A}-5 \mathrm{~B} \\
\text { [PUREX/WHC] } \\
(\text { A545,A546) }\end{array}$ & 17.1 & HEPA & $2.8 \mathrm{E}+08$ & $\begin{array}{l}\text { total alpha } \\
\text { total beta }\end{array}$ & $\begin{array}{l}1.9 \mathrm{E}-16 \\
3.1 \mathrm{E}-16\end{array}$ & $\begin{array}{ll}5.4 & E-08 \\
8.8 & E-08\end{array}$ \\
\hline $\begin{array}{l}296-A-6 \\
\text { [PUREX/WHC] } \\
\text { (A547) }\end{array}$ & 20.7 & HEPA & $1.8 \mathrm{E}+08$ & $\begin{array}{l}\text { total alpha } \\
\text { total beta }\end{array}$ & $\begin{array}{c}\mathrm{ND} \\
5.6 \mathrm{E}-17\end{array}$ & $\begin{array}{c}\text { ND } \\
1.4 \mathrm{E}-08\end{array}$ \\
\hline $\begin{array}{l}296 \cdot A-7 \\
\text { [PUREX/WHC] } \\
\text { (A548) }\end{array}$ & 20.7 & HEPA & $2.7 \mathrm{E}+08$ & $\begin{array}{l}\text { total alpha } \\
\text { total beta }\end{array}$ & $\begin{array}{l}3.2 \mathrm{E}-17 \\
5.7 \text { E-16 }\end{array}$ & $\begin{array}{ll}1.2 & \mathrm{E}-08 \\
2.1 & \mathrm{E}-07\end{array}$ \\
\hline $\begin{array}{l}296-\mathrm{A}-8 \\
\text { [PUREX/WHC] } \\
\text { (A549) }\end{array}$ & 20.7 & HEPA & $2.1 \mathrm{E}+08$ & $\begin{array}{l}\text { total alpha } \\
\text { total beta }\end{array}$ & $\begin{array}{l}2.5 \mathrm{E}-16 \\
2.0 \mathrm{E}-15\end{array}$ & $\begin{array}{ll}7.1 & \text { E-08 } \\
5.7 & \text { E-07 }\end{array}$ \\
\hline $\begin{array}{l}296-\mathrm{A}-10 \\
\text { [PUREX/WHC] } \\
\text { (A550) }\end{array}$ & 4.6 & HEPA & $1.0 \mathrm{E}+08$ & $\begin{array}{l}\text { total alpha } \\
\text { total beta }\end{array}$ & $\begin{array}{l}\text { ND } \\
\text { ND }\end{array}$ & $\begin{array}{l}\text { ND } \\
\text { ND }\end{array}$ \\
\hline $\begin{array}{l}296-\mathrm{A}-14 \\
\text { [PUREX/WHC] } \\
\text { (A544) }\end{array}$ & 9.1 & HEPA & $6.9 \mathrm{E}+07$ & $\begin{array}{l}\text { total alpha } \\
\text { total beta }\end{array}$ & $\begin{array}{l}1.4 \mathrm{E}-16 \\
2.3 \mathrm{E}-16\end{array}$ & $\begin{array}{l}1.3 \mathrm{E}-08 \\
2.2 \mathrm{E}-08\end{array}$ \\
\hline $\begin{array}{l}\text { 296-A-24 } \\
\text { [PUREX/WHC] } \\
\text { (A539) }\end{array}$ & 24.0 & None & $0.0 \mathrm{E}+00$ & & fid not operate) & \\
\hline $\begin{array}{l}296-\mathrm{B}-5 \\
\text { [B Plant/WHC] } \\
\text { (B686) }\end{array}$ & 3.7 & HEPA & $2.2 \mathrm{E}+07$ & $\begin{array}{l}\text { total alpha } \\
\text { total beta }\end{array}$ & $\begin{array}{l}1.5 \mathrm{E}-16 \\
5.2 \mathrm{E}-16\end{array}$ & $\begin{array}{l}4.5 \mathrm{E}-09 \\
1.6 \mathrm{E}-08\end{array}$ \\
\hline
\end{tabular}


Table 2-3

(10 sheets)

\begin{tabular}{|c|c|c|c|c|c|c|}
\hline \multicolumn{7}{|c|}{$\begin{array}{l}1995 \text { Hanford Site Radionuclide Air Emissions Data } \\
\text { for Minor Point Sources from WHC and BHI Facilities. } \\
\text { (minor point sources have the potential of }<0.1 \text { mrem/y EDE to nearest offsite resident) }\end{array}$} \\
\hline $\begin{array}{l}\text { Source } \text { ID }^{\mathbf{a}} \\
\text { [Facility/Contractor] } \\
\text { (EDP Code(s)) }\end{array}$ & $\begin{array}{l}\text { Discharge } \\
\text { Height } \\
\text { (m) }\end{array}$ & $\begin{array}{l}\text { Emission } \\
\text { Contral }^{b}\end{array}$ & $\begin{array}{l}\text { Total } \\
\text { Flow } \\
\left(\mathbf{m}^{3}\right)\end{array}$ & Radionuclide & $\begin{array}{c}\text { Average } \\
\text { Concentration } \\
(\mu \mathrm{Ci} / \mathrm{mL})^{\mathrm{c}, \mathrm{d}, \mathrm{e}}\end{array}$ & $\begin{array}{l}\text { Annual } \\
\text { Emissions } \\
\text { (Ci) }^{c, d, e}\end{array}$ \\
\hline $\begin{array}{l}\text { 296-B-10 } \\
\text { [B Plant/WHC] } \\
\text { (B748) }\end{array}$ & 22.9 & HEPA & $3.0 \mathrm{E}+08$ & $\begin{array}{l}{ }^{90} \mathrm{Sr} \\
{ }^{106} \mathrm{Ru} \\
{ }^{125} \mathrm{Sb} \\
{ }^{137} \mathrm{Cs} \\
{ }^{152} \mathrm{Eu} \\
{ }^{154} \mathrm{Eu} \\
{ }^{155} \mathrm{Eu} \\
{ }^{238} \mathrm{Pu} \\
{ }^{239} / 240 \mathrm{Pu} \\
{ }^{241} \mathrm{Am} \\
\text { total alpha } \\
\text { total beta }\end{array}$ & $\begin{array}{c}1.5 \mathrm{E}-14 \\
5.4 \mathrm{E}-18 \\
<5.4 \mathrm{E}-17 \\
7.1 \mathrm{E}-15 \\
<3.9 \mathrm{E}-17 \\
<1.4 \mathrm{E}-16 \\
<2.7 \mathrm{E}-17 \\
\mathrm{ND} \\
1.3 \mathrm{E}-18 \\
3.2 \mathrm{E}-17 \\
4.8 \mathrm{E}-16 \\
3.5 \mathrm{E}-14\end{array}$ & $\begin{array}{l}6.0 \mathrm{E}-06 \\
2.2 \mathrm{E}-09 \\
2.2 \mathrm{E}-08 \\
2.9 \mathrm{E}-06 \\
1.6 \mathrm{E}-08 \\
5.8 \mathrm{E}-08 \\
1.1 \mathrm{E}-08 \\
\mathrm{ND} \\
5.2 \mathrm{E}-10 \\
1.3 \mathrm{E}-08 \\
2.0 \mathrm{E}-07 \\
1.4 \mathrm{E}-05\end{array}$ \\
\hline $\begin{array}{l}\text { 296-B-13 } \\
{[\text { B Plant/WHC] }} \\
\text { (B690) }\end{array}$ & 3.5 & HEPA & $1.0 \mathrm{E}+07$ & $\begin{array}{l}\text { total alpha } \\
\text { total beta }\end{array}$ & $\begin{array}{l}2.0 \mathrm{E}-16 \\
1.0 \mathrm{E}-15\end{array}$ & $\begin{array}{l}2.8 \mathrm{E}-09 \\
1.5 \mathrm{E}-08\end{array}$ \\
\hline $\begin{array}{l}296-\mathrm{A}-13 \\
\text { [East Tank Farms/WHC] } \\
\text { (E052) }\end{array}$ & 38.1 & HEPA & $0.0 E+00$ & \multicolumn{3}{|c|}{ (did not operate) } \\
\hline $\begin{array}{l}296-\mathrm{A}-18 \\
\text { [East Tank Farms/WHC] } \\
\text { (E060) }\end{array}$ & 4.6 & HEPA & $0.0 \mathrm{E}+00$ & \multicolumn{3}{|c|}{ (did not operate) } \\
\hline $\begin{array}{l}296-\mathrm{A}-19 \\
\text { [East Tank Farms/WHC] } \\
\text { (E061) }\end{array}$ & 4.6 & HEPA & $0.0 \mathrm{E}+00$ & \multicolumn{3}{|c|}{ (did not operate) } \\
\hline $\begin{array}{l}296-\mathrm{A}-20 \\
\text { [East Tank Farms/WHCl } \\
\text { (E197) }\end{array}$ & 7.3 & HEPA & $1.6 \mathrm{E}+07$ & $\begin{array}{l}\text { total alpha } \\
\text { total beta }\end{array}$ & $\begin{array}{l}5.5 \mathrm{E}-16 \\
1.6 \mathrm{E}-15\end{array}$ & $\begin{array}{ll}1.1 & \text { E-08 } \\
3.1 & \text { E-08 }\end{array}$ \\
\hline $\begin{array}{l}296-A-26 \\
\text { [East Tank Farms/WHC] } \\
\text { (E297) }\end{array}$ & 9.4 & HEPA & $2.9 \mathrm{E}+07$ & $\begin{array}{l}\text { total alpha } \\
\text { total beta }\end{array}$ & $\begin{array}{l}1.8 \mathrm{E}-16 \\
6.0 \mathrm{E}-16\end{array}$ & $\begin{array}{l}7.2 \text { E-09 } \\
2.4 \text { E-08 }\end{array}$ \\
\hline $\begin{array}{l}296-\mathrm{A}-27 \\
\text { [East Tank Farms/WHC] } \\
\text { (E270,E933,E934) }\end{array}$ & 3.7 & HEPA & $1.8 \mathrm{E}+07$ & $\begin{array}{l}{ }_{0}^{90} \mathrm{Sr} \\
{ }^{106} \mathrm{Ru} \\
{ }^{13} \mathrm{Sn} \\
{ }^{125} \mathrm{Sb} \\
{ }^{229} \mathrm{I} \\
{ }^{137} \mathrm{Cs} \\
{ }^{238} \mathrm{Pu} \\
{ }^{239 / 240} \mathrm{Pu} \\
{ }^{241} \mathrm{Am} \\
\text { total alpha } \\
\text { total beta }\end{array}$ & $\begin{array}{c}\text { ND } \\
6.2 \text { E-14 } \\
<5.7 \text { E-15 } \\
<1.4 \text { E-14 } \\
6.4 \text { E-13 } \\
7.2 \text { E-17 } \\
\text { ND } \\
4.7 \text { E-18 } \\
2.3 \text { E-17 } \\
2.2 \text { E-16 } \\
5.3 \text { E-14 }\end{array}$ & $\begin{array}{c}\text { ND } \\
1.3 \text { E-06 } \\
1.2 \text { E-07 } \\
3.5 \text { E-07 } \\
1.4 \text { E-05 } \\
2.5 \text { E-09 } \\
\text { ND } \\
1.7 \text { E-10 } \\
8.2 \text { E-10 } \\
7.9 \text { E-09 } \\
1.9 \mathrm{E}-06\end{array}$ \\
\hline
\end{tabular}


Table 2-3

(10 sheets)

\begin{tabular}{|c|c|c|c|c|c|c|}
\hline \multicolumn{7}{|c|}{$\begin{array}{l}1995 \text { Hanford Site Radionuclide Air Emissions Data } \\
\text { for Minor Point Sources from WHC and BHI Facilities. } \\
\text { (minor point sources have the potential of }<0.1 \mathrm{mrem} / \mathrm{y} \text { EDE to nearest offsite resident) }\end{array}$} \\
\hline $\begin{array}{l}\text { Source ID } \\
\text { [Facility/Contractor] } \\
\text { (EDP Code(s)) }\end{array}$ & $\begin{array}{l}\text { Discharge } \\
\text { Height } \\
\text { (m) }\end{array}$ & $\begin{array}{l}\text { Emission } \\
\text { Controlb }^{b}\end{array}$ & $\begin{array}{l}\text { Total } \\
\text { Flow } \\
\left(\mathbf{m}^{3}\right)\end{array}$ & Radionuclide & $\begin{array}{c}\text { Average } \\
\text { Concentration } \\
(\mu \mathrm{Ci} / \mathbf{m L})^{\mathrm{c}, \mathrm{d} e}\end{array}$ & $\begin{array}{l}\text { Annual } \\
\text { Emissions } \\
\text { (Ci) }{ }^{\text {c,di,e }}\end{array}$ \\
\hline $\begin{array}{l}296-\mathrm{A}-28 \\
\text { East Tank Farms/WHC] } \\
\text { (E272) }\end{array}$ & 3.7 & HEPA & $9.3 \mathrm{E}+07$ & $\begin{array}{l}\text { total alpha } \\
\text { total beta }\end{array}$ & $\begin{array}{l}2.3 \mathrm{E}-16 \\
2.0 \mathrm{E}-15\end{array}$ & $\begin{array}{l}4.6 \mathrm{E}-08 \\
3.9 \mathrm{E}-07\end{array}$ \\
\hline $\begin{array}{l}296-A-29 \\
\text { [East Tank Farms/WHC] } \\
\text { (E901) }\end{array}$ & 3.7 & HEPA & $1.4 \mathrm{E}+07$ & $\begin{array}{l}{ }^{90} \mathrm{Sr} \\
{ }^{137} \mathrm{Cs} \\
{ }^{154} \mathrm{Eu} \\
{ }^{238} \mathrm{Pu} \\
{ }^{239 / 240} \mathrm{Pu} \\
{ }^{241} \mathrm{Am} \\
\text { total alpha } \\
\text { total beta }\end{array}$ & $\begin{array}{c}7.8 \text { E-16 } \\
7.4 \text { E-18 } \\
<3.6 \text { E-17 } \\
1.1 \text { E-18 } \\
4.1 \text { E-18: } \\
4.1 \text { E-17 } \\
3.0 \text { E-16 } \\
3.0 \text { E-14 }\end{array}$ & $\begin{array}{l}2.0 \mathrm{E}-08 \\
1.9 \mathrm{E}-10 \\
9.4 \mathrm{E}-10 \\
3.0 \mathrm{E}-11 \\
1.1 \mathrm{E}-10 \\
1.1 \mathrm{E}-09 \\
8.0 \mathrm{E}-09 \\
7.9 \mathrm{E}-07\end{array}$ \\
\hline $\begin{array}{l}296-\mathrm{A}-30 \\
\text { [East Tank Farms/WHC] } \\
\text { (E903) }\end{array}$ & 3.7 & HEPA & $9.2 \mathrm{E}+07$ & $\begin{array}{l}\text { total alpha } \\
\text { total beta }\end{array}$ & $\begin{array}{l}1.2 \mathrm{E}-16 \\
1.1 \mathrm{E}-15\end{array}$ & $\begin{array}{ll}2.1 & \mathrm{E}-08 \\
1.8 \mathrm{E}-07\end{array}$ \\
\hline $\begin{array}{l}\text { 296-A-41 } \\
\text { [East Tank Farms/WHC] } \\
\text { (E015) }\end{array}$ & 8.9 & HEPA & $1.5 \mathrm{E}+08$ & $\begin{array}{l}\text { total alpha } \\
\text { total beta }\end{array}$ & $\begin{array}{l}1.3 \mathrm{E}-16 \\
6.1 \mathrm{E}-16\end{array}$ & $\begin{array}{l}2.4 \text { E-08 } \\
1.1 \text { E-07 }\end{array}$ \\
\hline $\begin{array}{l}296-\text { P-17 } \\
\text { [East Tank Farms/WHC] } \\
\text { (E120) }\end{array}$ & 4.6 & HEPA & $0.0 \mathrm{E}+00$ & \multicolumn{3}{|c|}{ (did not operate) } \\
\hline $\begin{array}{l}296-P+31 \\
\text { [East Tank Farms/WHC] } \\
\text { (E209) }\end{array}$ & 10.0 & HEPA & $3.3 \mathrm{E}+07$ & $\begin{array}{l}\text { total alpha } \\
\text { total beta }\end{array}$ & $\begin{array}{l}1.8 \mathrm{E}-16 \\
1.2 \mathrm{E}-15\end{array}$ & $\begin{array}{l}6.4 \mathrm{E}-09 \\
4.2 \mathrm{E}-08\end{array}$ \\
\hline $\begin{array}{l}\text { 296-A-21 } \\
\text { [242-A Evaporator/WHC] } \\
\text { (E645) }\end{array}$ & 6.7 & HEPA & $3.4 \mathrm{E}+08$ & $\begin{array}{l}{ }^{90} \mathrm{Sr} \\
{ }_{106} \mathrm{Ru} \\
{ }^{125} \mathrm{Sb} \\
{ }^{137} \mathrm{Cs} \\
{ }^{152} \mathrm{Eu} \\
{ }^{154} \mathrm{Eu} \\
{ }^{155} \mathrm{Eu} \\
{ }^{238} \mathrm{Pu} \\
{ }^{239 / 240} \mathrm{Pu} \\
{ }^{241} \mathrm{Am} \\
\text { total alpha } \\
\text { total beta }\end{array}$ & $\begin{array}{c}1.7 \text { E-15 } \\
9.3 \text { E- } 17 \\
<1.8 \text { E-17 } \\
2.0 \text { E-17 } \\
<5.4 \text { E-18 } \\
<1.7 \text { E-16 } \\
<3.7 \text { E-18 } \\
2.7 \text { E-18 } \\
3.4 \text { E-17 } \\
2.5 \text { E-17 } \\
1.2 \text { E-16 } \\
8.2 \text { E-16 }\end{array}$ & $\begin{array}{ll}7.3 & \text { E-07 } \\
4.0 & \text { E-08 } \\
7.6 & \text { E-09 } \\
8.5 & \text { E-09 } \\
2.3 & \text { E-09 } \\
7.1 & \text { E-08 } \\
1.6 & \text { E-09 } \\
1.1 & \text { E-09 } \\
1.4 & \text { E-08 } \\
1.1 & \text { E-08 } \\
5.1 & \text { E-08 } \\
3.5 & \text { E-07 }\end{array}$ \\
\hline $\begin{array}{l}296-\mathrm{E}-1 \\
{[\mathrm{ETF} / \mathrm{WHC}]} \\
(\mathrm{E} 036)\end{array}$ & 15.5 & HEPA & $1.2 \mathrm{E}+08$ & $\begin{array}{l}\text { total alpha } \\
\text { total beta }\end{array}$ & $\begin{array}{l}6.0 \mathrm{E}-16 \\
1.3 \mathrm{E}-15\end{array}$ & $\begin{array}{l}9.6 \mathrm{E}-08 \\
2.1 \mathrm{E}-07\end{array}$ \\
\hline $\begin{array}{l}296-\mathrm{G}-1 \\
{[\mathrm{Grout} / \mathrm{WHC}]} \\
(\mathrm{E} 032)\end{array}$ & 7.6 & HEPA & $0.0 \mathrm{E}+00$ & \multicolumn{3}{|c|}{ (did not operate) } \\
\hline
\end{tabular}


Table 2-3

\begin{tabular}{|c|c|c|c|c|c|c|}
\hline \multicolumn{7}{|c|}{$\begin{array}{l}1995 \text { Hanford Site Radionuclide Air Emissions Data } \\
\text { for Minor Point Sources from WHC and BHI Facilities. } \\
\text { (minor point sources have the potential of }<0.1 \mathrm{mrem} / \mathrm{y} \text { EDE to nearest offsite resident) }\end{array}$} \\
\hline $\begin{array}{l}\text { Source } \text { ID }^{\text {a }} \\
\text { [Facility/Contractor] } \\
\text { (EDP Code(s)) }\end{array}$ & $\begin{array}{c}\text { Discharge } \\
\text { Height } \\
\text { (m) }\end{array}$ & $\begin{array}{l}\text { Emission } \\
\text { Control }^{\mathrm{b}}\end{array}$ & $\begin{array}{l}\text { Total } \\
\text { Flow } \\
\left(\mathbf{m}^{3}\right)\end{array}$ & Radionuclide & $\begin{array}{l}\text { Average } \\
\text { Concentration } \\
(\mu \mathrm{Ci} / \mathrm{mL})^{c, d, e}\end{array}$ & $\begin{array}{c}\text { Annual } \\
\text { Emissions } \\
(\text { Ci })^{c, d, e}\end{array}$ \\
\hline \multicolumn{7}{|c|}{200 West Area Point Sources } \\
\hline $\begin{array}{l}\text { 296-P-22 } \\
\text { [West Tank Farms/WHC] } \\
\text { (W191) }\end{array}$ & 4.6 & HEPA & $1.8 \mathrm{E}+07$ & $\begin{array}{l}\text { total aipha } \\
\text { total beta }\end{array}$ & $\begin{array}{l}7.8 \text { E-17 } \\
8.3 \text { E-16 }\end{array}$ & $\begin{array}{l}1.7 \mathrm{E}-09 \\
1.8 \mathrm{E}-08\end{array}$ \\
\hline $\begin{array}{l}296-P-23 \\
296-P-28 \text { (backup) } \\
\text { [West Tank Farms/WHC] } \\
\text { (W190,W195) }\end{array}$ & 4.6 & HEPA & $1.8 \mathrm{E}+07$ & $\begin{array}{l}{ }^{90} \mathrm{Sr} \\
{ }^{106} \mathrm{Ru} \\
{ }^{113} \mathrm{Sn} \\
{ }^{125} \mathrm{Sb} \\
{ }^{134} \mathrm{Cs} \\
{ }^{137} \mathrm{Cs} \\
{ }^{132} \mathrm{Eu} \\
{ }^{159} \mathrm{Eu} \\
{ }^{238} \mathrm{Pu} \\
{ }^{239} / 240 \mathrm{Pu} \\
{ }^{24)} \mathrm{Am} \\
\text { total alpha } \\
\text { total beta }\end{array}$ & $\begin{array}{c}7.2 \text { E-15 } \\
2.2 \text { E-16 } \\
<3.3 \text { E-17 } \\
<1.9 \text { E-16 } \\
2.2 \text { E-17 } \\
2.2 \text { E-13 } \\
<9.4 \text { E-17 } \\
<1.3 \text { E-16 } \\
2.2 \text { E-17 } \\
9.4 \text { E-17 } \\
2.2 \text { E-16 } \\
6.1 \text { E-16 } \\
1.6 \text { E-13 }\end{array}$ & $\begin{array}{l}1.3 \mathrm{E}-07 \\
4.0 \mathrm{E}-09 \\
6.0 \mathrm{E}-10 \\
3.4 \mathrm{E}-09 \\
4.0 \mathrm{E}-10 \\
4.0 \mathrm{E}-06 \\
1.7 \mathrm{E}-09 \\
2.4 \mathrm{E}-09 \\
3.9 \mathrm{E}-10 \\
1.7 \mathrm{E}-09 \\
3.9 \mathrm{E}-09 \\
1.1 \mathrm{E}-08 \\
2.9 \mathrm{E}-06\end{array}$ \\
\hline $\begin{array}{l}296-S-18 \\
\text { [West Tank Farms/WHC] } \\
\text { (W096) }\end{array}$ & 6.7 & HEPA & $2.2 \mathrm{E}+08$ & $\begin{array}{l}\text { total alpha } \\
\text { total beta }\end{array}$ & $\begin{array}{l}1.2 \mathrm{E}-16 \\
2.0 \mathrm{E}-15\end{array}$ & $\begin{array}{l}3.8 \mathrm{E}-08 \\
6.4 \mathrm{E}-07\end{array}$ \\
\hline $\begin{array}{l}296-\mathrm{T}-17 \\
\text { [West Tank Farms } \backslash \text { WHC] } \\
\text { (W } 117 \text { ) }\end{array}$ & 10.1 & HEPA & $3.0 \mathrm{E}+07$ & $\begin{array}{l}\text { total alpha } \\
\text { total beta }\end{array}$ & $\begin{array}{ll}6.0 & \mathrm{E}-17 \\
5.1 & \mathrm{E}-16\end{array}$ & $\begin{array}{ll}2.2 & \mathrm{E}-09 \\
2.8 & \mathrm{E}-08\end{array}$ \\
\hline $\begin{array}{l}296-\text { W-3 } \\
\text { [West Tank FarmslWHC] } \\
\text { (W003) }\end{array}$ & 7.6 & HEPA & $3.1 \mathrm{E}+07$ & $\begin{array}{l}\text { total alpha } \\
\text { total beta }\end{array}$ & $\begin{array}{l}3.2 \mathrm{E}-16 \\
1.4 \mathrm{E}-15\end{array}$ & $\begin{array}{l}1.4 \mathrm{E}-08 \\
5.9 \mathrm{E}-08\end{array}$ \\
\hline $\begin{array}{l}\text { 291-S-1 } \\
\text { [S Plant/BHI] } \\
(\mathrm{S} 006)\end{array}$ & 61.0 & sand filter & $3.1 \mathrm{E}+08$ & $\begin{array}{l}\text { total alpha } \\
\text { total beta }\end{array}$ & $\begin{array}{l}5.0 \mathrm{E}-16 \\
1.6 \mathrm{E}-15\end{array}$ & $\begin{array}{ll}2.1 & \text { E-07 } \\
6.7 & \text { E-07 }\end{array}$ \\
\hline $\begin{array}{l}296-\mathrm{S}-2 \\
\text { [S Plant/BHI] } \\
\text { (S032) }\end{array}$ & 20.7 & HEPA & $1.9 \mathrm{E}+07$ & $\begin{array}{l}\text { total alpha } \\
\text { total beta }\end{array}$ & $\begin{array}{l}2.6 \mathrm{E}-16 \\
6.0 \mathrm{E}-16\end{array}$ & $\begin{array}{l}6.9 \mathrm{E}-09 \\
1.6 \mathrm{E}-08\end{array}$ \\
\hline $\begin{array}{l}296-\mathrm{S}-4 \\
\text { [S Plant/BHI] } \\
(\mathrm{SO08})\end{array}$ & 20.7 & HEPA & $4.4 \mathrm{E}+06$ & $\begin{array}{l}\text { total alpha } \\
\text { total beta }\end{array}$ & $\begin{array}{ll}7.3 & \text { E-16 } \\
2.3 & \text { E-15 }\end{array}$ & $\begin{array}{l}4.4 \mathrm{E}-09 \\
1.4 \mathrm{E}-08\end{array}$ \\
\hline $\begin{array}{l}296-S-6 \\
\text { [S Plant/BHI] } \\
\text { (SO04) }\end{array}$ & 39.4 & none & $1.2 \mathrm{E}+07$ & $\begin{array}{l}\text { total alpha } \\
\text { total beta }\end{array}$ & $\begin{array}{l}1.3 \text { E-15 } \\
4.8 \text { E-15 }\end{array}$ & $\begin{array}{ll}2.2 & \text { E-08 } \\
8.0 & \text { E-08 }\end{array}$ \\
\hline
\end{tabular}


Table 2-3

(10 sheets)

\begin{tabular}{|c|c|c|c|c|c|c|}
\hline \multicolumn{7}{|c|}{$\begin{array}{l}1995 \text { Hanford Site Radionuclide Air Emissions Data } \\
\text { for Minor Point Sources from WHC and BHI Facilities. } \\
\text { (minor point sources have the potential of }<0.1 \text { mrem/y EDE to nearest offsite resident) }\end{array}$} \\
\hline $\begin{array}{l}\text { Source ID" } \\
\text { [Facility/Contractor] } \\
\text { (EDP Code(s)) }\end{array}$ & $\begin{array}{c}\text { Discharge } \\
\text { Height } \\
\text { (m) }\end{array}$ & $\begin{array}{l}\text { Emission } \\
\text { Control }^{b}\end{array}$ & $\begin{array}{l}\text { Total } \\
\text { Flow } \\
\left(\mathbf{m}^{3}\right)\end{array}$ & Radionuclide & $\begin{array}{l}\text { Average } \\
\text { Concentration } \\
(\mu \mathrm{Ci} / \mathbf{m L})^{\mathrm{c}, \mathrm{d}, e}\end{array}$ & $\begin{array}{c}\text { Annual } \\
\text { Emissions } \\
(\mathbf{C i})^{c, d, e}\end{array}$ \\
\hline $\begin{array}{l}\text { 296-S-7W } \\
\text { 296-S-7E (backup) } \\
\text { [S Plant/BHI] } \\
\text { (S015,S016) }\end{array}$ & 7.6 & HEPA & $1.4 \mathrm{E}+08$ & $\begin{array}{l}\text { total alpha } \\
\text { total beta }\end{array}$ & $\begin{array}{l}4.1 \text { E-15 } \\
2.1 \text { E-15 }\end{array}$ & $\begin{array}{l}5.6 \text { E }-07 \\
2.9 \text { E-07 }\end{array}$ \\
\hline $\begin{array}{l}291-\mathrm{U}-1 \\
\text { [U Plant/BHI] } \\
\text { (U771) }\end{array}$ & 61.0 & sand filter & $3.7 E+08$ & $\begin{array}{l}\text { total alpha } \\
\text { total beta }\end{array}$ & $\begin{array}{l}5.0 \mathrm{E}-16 \\
1.1 \mathrm{E}-13\end{array}$ & $\begin{array}{l}2.5 \mathrm{E}-07 \\
5.4 \mathrm{E}-05\end{array}$ \\
\hline $\begin{array}{l}291-\mathrm{T}-1 \\
\text { [T Plant/WHC] } \\
\text { (T785) }\end{array}$ & 61.0 & HEPA & $5.2 \mathrm{E}+08$ & $\begin{array}{l}{ }^{90} \mathrm{Sr} \\
{ }^{125} \mathrm{Sb} \\
{ }^{134} \mathrm{Cs} \\
{ }^{137} \mathrm{Cs} \\
{ }^{152} \mathrm{Eu} \\
{ }^{154} \mathrm{Eu} \\
{ }^{155} \mathrm{Eu} \\
{ }^{238} \mathrm{Pu} \\
{ }^{239 i 240} \mathrm{Pu} \\
{ }^{241} \mathrm{Am} \\
\text { total alpha } \\
\text { total beta }\end{array}$ & $\begin{array}{c}3.7 \text { E-14 } \\
<2.8 \text { E-17 } \\
2.4 \text { E-17 } \\
2.1 \text { E-14 } \\
<7.2 \text { E-17 } \\
<1.9 \text { E-16 } \\
<1.5 \text { E-16 } \\
4.5 \text { E-16 } \\
3.4 \text { E-14 } \\
2.5 \text { E-15 } \\
7.2 \text { E-14 } \\
2.2 \text { E-13 }\end{array}$ & $\begin{array}{ll}2.7 & \mathrm{E}-05 \\
2.0 & \mathrm{E}-08 \\
1.7 & \mathrm{E}-08 \\
1.5 & \mathrm{E}-05 \\
5.2 & \mathrm{E}-08 \\
1.3 & \mathrm{E}-07 \\
1.1 & \mathrm{E}-07 \\
3.2 & \mathrm{E}-07 \\
2.4 & \mathrm{E}-05 \\
1.8 & \mathrm{E}-06 \\
5.2 & \mathrm{E}-05 \\
1.6 & \mathrm{E}-04\end{array}$ \\
\hline $\begin{array}{l}\text { 296-T-7 } \\
\text { [T Plant/WHC] } \\
\text { (T154) }\end{array}$ & 8.5 & HEPA & $2.3 \mathrm{E}+07$ & $\begin{array}{l}\text { total alpha } \\
\text { total beta }\end{array}$ & $\begin{array}{l}1.5 \mathrm{E}-16 \\
1.4 \mathrm{E}-15\end{array}$ & $\begin{array}{l}5.0 \mathrm{E}-09 \\
4.6 \mathrm{E}-08\end{array}$ \\
\hline $\begin{array}{l}296-\mathrm{T}-13 \\
{[\mathrm{~T} \text { Plant/WHC] }} \\
(\mathrm{T} 786)\end{array}$ & 20.7 & HEPA & $5.6 \mathrm{E}+08$ & $\begin{array}{l}\text { total alpha } \\
\text { total beta }\end{array}$ & $\begin{array}{l}1.5 \mathrm{E}-15 \\
1.2 \mathrm{E}-14\end{array}$ & $\begin{array}{l}1.1 \text { E-06 } \\
8.8 \text { E-06 }\end{array}$ \\
\hline $\begin{array}{l}\text { 296-T-1] } \\
\text { [TRUSAF/WHC] } \\
\text { (T783) }\end{array}$ & 7.6 & HEPA & $5.8 \mathrm{E}+06$ & $\begin{array}{l}\text { total alpha } \\
\text { total beta }\end{array}$ & $\begin{array}{l}9.8 \mathrm{E}-17 \\
4.7 \text { E-16 }\end{array}$ & $\begin{array}{l}7.8 \text { E-10 } \\
3.8 \text { E-09 }\end{array}$ \\
\hline $\begin{array}{l}\text { 296-T-12 } \\
\text { [TRUSAF/WHC] } \\
\text { (T784) }\end{array}$ & 7.6 & HEPA & $1.4 \mathrm{E}+08$ & $\begin{array}{l}\text { total alpha } \\
\text { total beta }\end{array}$ & $\begin{array}{l}6.4 \text { E-16 } \\
4.1 \text { E-15 }\end{array}$ & $\begin{array}{l}1.2 \mathrm{E}-07 \\
7.9 \mathrm{E}-07\end{array}$ \\
\hline $\begin{array}{l}296-S-16 \\
{[222-S / W H C]} \\
(\$ 264)\end{array}$ & 3.0 & HEPA & $1.6 \mathrm{E}+06$ & $\begin{array}{l}\text { total alpha } \\
\text { total beta }\end{array}$ & $\begin{array}{l}1.2 \mathrm{E}-14 \\
2.5 \mathrm{E}-13\end{array}$ & $\begin{array}{ll}2.7 & \text { E-08 } \\
5.4 & \text { E-07 }\end{array}$ \\
\hline $\begin{array}{l}296-\mathrm{S}-21 \\
{[222-\mathrm{S} / \mathrm{WHC}]} \\
(\mathrm{S} 289)\end{array}$ & 11.6 & HEPA & $1.1 \mathrm{E}+09$ & $\begin{array}{l}{ }^{238} \mathrm{Pu} \\
{ }^{239} / 240 \mathrm{Pu} \\
{ }^{24 !} \mathrm{Am} \\
\text { total alpha } \\
\text { total beta }\end{array}$ & 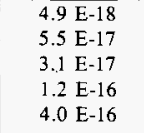 & $\begin{array}{ll}7.1 & \mathrm{E}-09 \\
8.0 & \mathrm{E}-08 \\
4.5 & \mathrm{E}-08 \\
1.8 & \mathrm{E}-07 \\
5.8 & \mathrm{E}-07\end{array}$ \\
\hline
\end{tabular}


Table 2-3

(10 sheets)

\begin{tabular}{|c|c|c|c|c|c|c|}
\hline \multicolumn{7}{|c|}{$\begin{array}{l}1995 \text { Hanford Site Radionuclide Air Emissions Data } \\
\text { for Minor Point Sources from WHC and BHI Facilities. } \\
\text { (minor point sources have the potential of }<0.1 \text { mrem/y EDE to nearest offsite resident) }\end{array}$} \\
\hline $\begin{array}{l}\text { Source ID } \\
\text { [Facility/Contractor] } \\
\text { (EDP Code(s)) }\end{array}$ & $\begin{array}{c}\text { Discharge } \\
\text { Height } \\
\text { (m) }\end{array}$ & $\begin{array}{l}\text { Emission } \\
\text { Control }\end{array}$ & $\begin{array}{l}\text { Total } \\
\text { Flow } \\
\left(\mathbf{m}^{3}\right)\end{array}$ & Radionuclide & $\begin{array}{l}\text { Average } \\
\text { Concentration } \\
(\mu \mathrm{Ci} / \mathbf{m L})^{\mathrm{c}, \boldsymbol{d}, \mathrm{e}}\end{array}$ & $\begin{array}{l}\text { Annual } \\
\text { Emissions } \\
(\text { Ci })^{c, d, e}\end{array}$ \\
\hline $\begin{array}{l}296-\mathrm{Z}-3 \\
{[\mathrm{PFP} / \mathrm{WHC}]} \\
(2813)\end{array}$ & 7.6 & HEPA & $1.4 \mathrm{E}+07$ & $\begin{array}{l}{ }^{238} \mathrm{Pu} \\
{ }^{239 / 240} \mathrm{Pu} \\
{ }^{2+1} \mathrm{Pu} \\
{ }^{241} \mathrm{Am} \\
\text { total alpha } \\
\text { total beta }\end{array}$ & $\begin{array}{l}1.8 \text { E-15 } \\
1.6 \text { E-15 } \\
1.6 \text { E-13 } \\
7.9 \text { E-16 } \\
4.3 \text { E-15 } \\
2.1 \text { E-15 }\end{array}$ & $\begin{array}{ll}3.5 & \mathrm{E}-08 \\
3.1 & \mathrm{E}-08 \\
3.1 & \mathrm{E}-06 \\
1.5 & \mathrm{E}-08 \\
8.1 & \mathrm{E}-08 \\
4.0 & \mathrm{E}-08\end{array}$ \\
\hline $\begin{array}{l}296-\mathrm{Z}-5 \\
\text { [PFP/WHC] } \\
(\mathrm{Z} 913)\end{array}$ & 4.6 & HEPA & $1.4 \mathrm{E}+08$ & $\begin{array}{l}\text { total alpha } \\
\text { total beta }\end{array}$ & $\begin{array}{l}9.9 \mathrm{E}-17 \\
3.0 \mathrm{E}-16\end{array}$ & $\begin{array}{l}1.9 \mathrm{E}-08 \\
5.6 \mathrm{E}-08\end{array}$ \\
\hline $\begin{array}{l}296-\mathrm{Z}-6 \\
{[\mathrm{PFP} / \mathrm{WHC}]} \\
(\mathrm{Z} 802)\end{array}$ & 0.9 & HEPA & $1.3 \mathrm{E}+08$ & $\begin{array}{l}\text { total alpha } \\
\text { total beta }\end{array}$ & 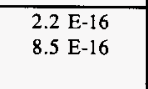 & $\begin{array}{ll}4.1 & \mathrm{E}-08 \\
1.6 & \mathrm{E}-07\end{array}$ \\
\hline $\begin{array}{l}296-Z-14 \\
{[\mathrm{PFP} / W H C]} \\
(\mathrm{Z} 814)\end{array}$ & 6.1 & HEPA & $3.0 \mathrm{E}+07$ & $\begin{array}{l}\text { total alpha } \\
\text { total beta }\end{array}$ & $\begin{array}{ll}2.1 & \text { E-16 } \\
1.2 & \text { E-15 }\end{array}$ & $\begin{array}{l}8.6 \mathrm{E}-09 \\
4.9 \mathrm{E}-08\end{array}$ \\
\hline $\begin{array}{l}296-Z-15 \\
{[\mathrm{PFP} / \mathrm{WHC}]} \\
(\mathrm{Z} 915)\end{array}$ & 3.6 & HEPA & $2.4 \mathrm{E}+07$ & total alpha & $1.2 \mathrm{E}-16$ & $3.9 \mathrm{E}-09$ \\
\hline $\begin{array}{l}\text { 696-W-1 } \\
\text { [WSCFIWHC] } \\
\text { (W010) }\end{array}$ & 7.6 & HEPA & $7.4 \mathrm{E}+08$ & $\begin{array}{l}\text { total alpha } \\
\text { total beta }\end{array}$ & $\begin{array}{l}9.2 \text { E-17 } \\
4.9 \text { E-16 }\end{array}$ & $\begin{array}{l}9.4 \mathrm{E}-08 \\
5.0 \mathrm{E}-07\end{array}$ \\
\hline $\begin{array}{l}\text { 696-W-2 } \\
\text { [WSCF/WHC] } \\
\text { (W011) }\end{array}$ & 9.8 & HEPA & $2.2 \mathrm{E}+07$ & $\begin{array}{l}\text { total alpha } \\
\text { total beta }\end{array}$ & $\begin{array}{l}2.0 \mathrm{E}-16 \\
1.2 \mathrm{E}-15\end{array}$ & $\begin{array}{ll}6.1 & \mathrm{E}-09 \\
3.6 & \mathrm{E}-08\end{array}$ \\
\hline \multicolumn{7}{|c|}{300 Area Point Sources } \\
\hline $\begin{array}{l}\text { 306-E-ULAB } \\
\text { [306-E Bldg./WHC] } \\
\text { (F003) }\end{array}$ & 20.1 & HEPA & $4.4 \mathrm{E}+07$ & $\begin{array}{l}\text { total alpha } \\
\text { total beta }\end{array}$ & $\begin{array}{l}3.6 \text { E-16 } \\
8.4 \text { E-16 }\end{array}$ & $\begin{array}{l}2.2 \text { E-08 } \\
5.0 \text { E-08 }\end{array}$ \\
\hline $\begin{array}{l}\text { 308-TRIGA } \\
\text { [308 Bldg./WHC] } \\
\text { (F006) }\end{array}$ & 11.3 & $\begin{array}{l}\text { HEPA. } \\
\text { charcoal }\end{array}$ & $2.7 \mathrm{E}+07$ & $\begin{array}{l}{ }^{131} \mathrm{I} \\
\text { total alpha } \\
\text { total beta }\end{array}$ & $\begin{array}{c}\mathrm{ND} \\
1.4 \mathrm{E}-15 \\
3.2 \mathrm{E}-15\end{array}$ & $\begin{array}{c}\mathrm{ND} \\
5.3 \mathrm{E}-08 \\
1.2 \mathrm{E}-07\end{array}$ \\
\hline $\begin{array}{l}\text { 309-PRTR } \\
{[309 \text { Bldg./WHC] }}\end{array}$ & 30.5 & HEPA & $3.5 \mathrm{E}+07$ & $\begin{array}{l}{ }^{60} \mathrm{Co} \\
{ }^{90} \mathrm{Sr} \\
{ }_{137}^{137} \mathrm{Cs} \\
{ }^{239} \mathrm{Pu}\end{array}$ & $\begin{array}{l}\text { ND } \\
\text { ND } \\
\text { ND } \\
\text { ND }\end{array}$ & $\begin{array}{l}<3.7 \text { E-10 } \\
<1.4 \text { E-10 } \\
<3.3 \text { E-10 } \\
<1.2 \text { E-10 }\end{array}$ \\
\hline $\begin{array}{l}\text { 309-RTF-EX } \\
{[309 \text { Bldg } / \text { WHC] }}\end{array}$ & 4.0 & HEPA & $4.8 \mathrm{E}+06$ & ${ }^{137} \mathrm{Cs}$ & $\overline{N D}$ & $<6.3 \mathrm{E}-12$ \\
\hline $\begin{array}{l}\text { 340-B-BLDG } \\
{[340 \text { Bldg. } / \text { WHC] }} \\
\text { (F008) }\end{array}$ & 11.6 & HEPA & $5.0 E+06$ & $\begin{array}{l}\text { total alpha } \\
\text { total beta }\end{array}$ & $\begin{array}{l}\text { ND } \\
\text { ND }\end{array}$ & $\begin{array}{l}\mathrm{ND} \\
\mathrm{ND}\end{array}$ \\
\hline
\end{tabular}


Table 2-3

(10 sheets)

\begin{tabular}{|c|c|c|c|c|c|c|}
\hline \multicolumn{7}{|c|}{$\begin{array}{l}1995 \text { Hanford Site Radionuclide Air Emissions Data } \\
\text { for Minor Point Sources from WHC and BHI Facilities. } \\
\text { (minor point sources have the potential of }<0.1 \mathrm{mrem} / \mathrm{y} \text { EDE to nearest offsite resident) }\end{array}$} \\
\hline $\begin{array}{l}\text { Source ID } \\
\text { [Facility/Contractor] } \\
\text { (EDP Code(s)) }\end{array}$ & $\begin{array}{l}\text { Discharge } \\
\text { Height } \\
\text { (m) }\end{array}$ & $\begin{array}{l}\text { Emission } \\
\text { Control }^{\mathrm{b}}\end{array}$ & $\begin{array}{l}\text { Total } \\
\text { Flow } \\
\left(\mathbf{m}^{3}\right)\end{array}$ & Radionuclide & $\begin{array}{l}\text { Average } \\
\text { Concentration } \\
(\mu \mathrm{Ci} / \mathrm{mL})^{\mathrm{c}, \mathrm{d}, \mathrm{e}}\end{array}$ & $\begin{array}{l}\text { Annual } \\
\text { Emissions } \\
\text { (Ci) }\end{array}$ \\
\hline $\begin{array}{l}\text { 340-DECON } \\
{[340 \text { Bldg./WHC] }} \\
(\mathrm{F} 009)\end{array}$ & 3.0 & HEPA & $1.1 \mathrm{E}+08$ & $\begin{array}{l}\text { total alpha } \\
\text { total beta }\end{array}$ & $\begin{array}{l}1.8 \text { E- } 15 \\
5.8 \text { E-15 }\end{array}$ & $\begin{array}{ll}2.7 & \mathrm{E}-07 \\
8.5 & \mathrm{E}-07\end{array}$ \\
\hline $\begin{array}{l}\text { 377-GEL } \\
{[377 \text { Bldg./WHC] }} \\
(\mathrm{F} 010)\end{array}$ & 10.0 & HEPA & $1.2 E+08$ & $\begin{array}{l}\text { total alpha } \\
\text { total beta }\end{array}$ & $\begin{array}{l}\text { ND } \\
\text { ND }\end{array}$ & $\begin{array}{l}\text { ND } \\
\text { ND }\end{array}$ \\
\hline \multicolumn{7}{|c|}{400 Area Point Sources } \\
\hline $\begin{array}{l}\text { FFTF-CB-EX } \\
\text { [FFTF/WHC] } \\
\text { (F011) }\end{array}$ & 14.3 & none & $3.2 \mathrm{E}+08$ & $\begin{array}{l}{ }^{3} \mathrm{H} \text { (as HTO) } \\
{ }^{131} \mathrm{I} \\
\text { total alpha } \\
\text { total beta }\end{array}$ & $\begin{array}{l}6.7 \text { E-11 } \\
\text { ND } \\
1.1 \text { E-15 } \\
3.3 \text { E-15 }\end{array}$ & $\begin{array}{c}2.5 \mathrm{E}-02 \\
\mathrm{ND} \\
4.9 \mathrm{E}-07 \\
1.4 \mathrm{E}-06\end{array}$ \\
\hline $\begin{array}{l}\text { FFTF-RE-SB } \\
\text { [FFTF/WHC] } \\
(\mathrm{F} 012)\end{array}$ & 6.1 & none & $2.7 \mathrm{E}+08$ & $\begin{array}{l}{ }^{131} I \\
\text { total alpha } \\
\text { total beta }\end{array}$ & $\begin{array}{l}\text { ND } \\
2.1 \text { E-15 } \\
8.5 \text { E-15 }\end{array}$ & $\begin{array}{c}\text { ND } \\
8.1 \text { E-07 } \\
3.2 \text { E-06 }\end{array}$ \\
\hline $\begin{array}{l}\text { FFTF-HT-TR } \\
\text { [FFTF/WHC] } \\
\text { (F013) }\end{array}$ & 8.8 & none & $6.7 \mathrm{E}+07$ & $\begin{array}{l}\text { total alpha } \\
\text { total beta }\end{array}$ & $\begin{array}{l}1.2 \mathrm{E}-15 \\
3.8 \mathrm{E}-15\end{array}$ & $\begin{array}{ll}1.1 & \text { E-07 } \\
3.5 & \text { E-07 }\end{array}$ \\
\hline $\begin{array}{l}\text { 437-MN\&ST } \\
{[\mathrm{MASF} / \mathrm{WHC}]} \\
(\mathrm{F} 014)\end{array}$ & 9.1 & none & $2.1 \mathrm{E}+08$ & $\begin{array}{l}\text { total alpha } \\
\text { total beta }\end{array}$ & $\begin{array}{l}1.1 \mathrm{E}-15 \\
3.3 \mathrm{E}-15\end{array}$ & $\begin{array}{l}3.2 \mathrm{E}-07 \\
9.5 \mathrm{E}-07\end{array}$ \\
\hline $\begin{array}{l}437-1-61 \\
\text { [MASF/WHC] } \\
\text { (F019) }\end{array}$ & 11.7 & HEPA & $2.4 \mathrm{E}+08$ & $\begin{array}{l}\text { total alpha } \\
\text { total beta }\end{array}$ & $\begin{array}{l}\text { ND } \\
\text { ND }\end{array}$ & $\begin{array}{l}\mathrm{ND} \\
\mathrm{ND}\end{array}$ \\
\hline
\end{tabular}

Notes:

a ID = Identification. i.e., the alpha-numeric designator for the respective point source; WHC = Westinghouse Hanford Company; PNNL = Pacific Northwest National Laboratory (Battelle); BHI = Bechtel Hanford. Inc.

b Efficiencies are: $\geq 99.95 \%$ for HEPA; $\geq 95 \%$ for charcoal: $\geq 99.8 \%$ for sand filter; $0 \%$ for no emission control.

c 1 Curie $=3.7 \mathrm{E}+10$ becquerel; $\mathrm{ND}=$ none detected (i.e. either the radionuclide was not detected in any sample during the year, or the average of all the measurements for that given radionuclide or type of radioactivity made during the year was below background levels).

d Note that the average concentrations do not have sampling system correction factors applied to them. Sampling system correction factors have been applied to the annual emissions estimates.

e $<=$ less than detectabie: Note that all 200 East and West Area results for gamma emitters ${ }^{113} \mathrm{Sn},{ }^{125} \mathrm{Sb},{ }^{152} \mathrm{Eu}$, ${ }^{154} \mathrm{Eu}$, and ${ }^{155} \mathrm{Eu}$ may have been false positives. since the gamma energy analyses did not identify any energy peaks for these isotopes. These results have been reported, causing emissions estimates to be conservatively higher than they really were. This problem will be corrected, such that false positives are not reported and that emissions estimates are more accurate. 
WHC-EP-0527-5

Table 2-4

\begin{tabular}{|c|c|c|c|}
\hline \multicolumn{4}{|c|}{$\begin{array}{l}1995 \text { Nonradioactive Air Emissions } \\
\text { from Hanford's Powerhouse Boilers }\end{array}$} \\
\hline & & Source & \\
\hline Constituent & $\begin{array}{l}200 \text { East Area } \\
\text { Powerhouse }\end{array}$ & $\begin{array}{l}200 \text { West Area } \\
\text { Powerhouse }\end{array}$ & $\begin{array}{l}300 \text { Area } \\
\text { Powerhouse }\end{array}$ \\
\hline Criteria Air Pollutants & \multicolumn{3}{|c|}{ Release, $\mathbf{k g}^{\mathbf{a}}$} \\
\hline Particulate matter & $1.70 \mathrm{E}+03$ & $3.19 \mathrm{E}+02$ & $1.60 \mathrm{E}+04$ \\
\hline Nitrogen oxides & $1.77 \mathrm{E}+05$ & $2.82 \mathrm{E}+04$ & $4.69 E+04$ \\
\hline Sulfur oxides & $2.25 E+05$ & $3.53 \mathrm{E}+04$ & $2.34 \mathrm{E}+05$ \\
\hline Carbon monoxide & $6.43 E+04$ & $1.01 \mathrm{E}+04$ & $4.25 \mathrm{E}+03$ \\
\hline Lead & $1.62 \mathrm{E}+02$ & $2.53 \mathrm{E}+01$ & $2.52 \mathrm{E}+01$ \\
\hline Volatile organic compounds & $6.43 \mathrm{E}+02$ & $1.00 \mathrm{E}+02$ & $2.38 \mathrm{E}+02$ \\
\hline Toxic Air Pollutants & \multicolumn{3}{|c|}{ Release, $\mathbf{k g}^{\mathbf{a}}$} \\
\hline Arsenic & $1.73 \mathrm{E}+02$ & $2.70 \mathrm{E}+01$ & $1.48 \mathrm{E}+01$ \\
\hline Beryllium & $2.33 \mathrm{E}+01$ & $3.64 \mathrm{E}+00$ & $5.46 \mathrm{E}-01$ \\
\hline Cadmium & $1.37 \mathrm{E}+01$ & $2.18 \mathrm{E}+00$ & $2.74 \mathrm{E}+01$ \\
\hline Chromium & $5.01 \mathrm{E}+02$ & $7.83 E+01$ & $1.67 \mathrm{E}+01$ \\
\hline Cobalt & NE & NE & $1.57 \mathrm{E}+01$ \\
\hline Copper & $3.15 \mathrm{E}+02$ & $5.02 \mathrm{E}+02$ & $3.62 \mathrm{E}+01$ \\
\hline Formaldehyde & $7.05 \mathrm{E}+01$ & $1.25 \mathrm{E}+01$ & $5.27 \mathrm{E}+01$ \\
\hline Manganese & $6.93 \mathrm{E}+02$ & $1.08 \mathrm{E}+02$ & $9.63 \mathrm{E}+00$ \\
\hline Mercury & $5.11 \mathrm{E}+00$ & $8.08 \mathrm{E}-01$ & $4.16 \mathrm{E}+00$ \\
\hline Nickel & $4.12 \mathrm{E}+02$ & $6.43 \mathrm{E}+01$ & $3.03 E+02$ \\
\hline Polycyclic organic matter & $\mathrm{NE}$ & $6.00 \mathrm{E}+02$ & $7.14 \mathrm{E}+03$ \\
\hline Selenium & $6.26 \mathrm{E}+01$ & $9.84 \mathrm{E}+00$ & $4.94 \mathrm{E}+00$ \\
\hline Vanadium & $4.31 \mathrm{E}+01$ & $7.79 \mathrm{E}+00$ & $3.93 \mathrm{E}+02$ \\
\hline Fuel Consumed & \multicolumn{3}{|c|}{ Total Fuel Consumption } \\
\hline $\begin{array}{l}\text { Coal. kg } \\
\text { Grade } 2 \text { Oil, L } \\
\text { Grade } 6 \text { Oil. L }\end{array}$ & $2.58 \mathrm{E}+07$ & $\begin{array}{l}4.02 \mathrm{E}+06 \\
2.28 \mathrm{E}+05\end{array}$ & $6.93 \mathrm{E}+06$ \\
\hline
\end{tabular}

a Releases calculated from EPA emission factors and based on total fuel consumption. 


\subsection{LIQUID EFFLUENTS}

The majority of liquid effluents released to the environment from facilities managed by WHC and facilities monitored by BHI were discharged to the soil at the Hanford Site, with the remainder being discharged to the Columbia River. Data on the 1995 radioactive and nonradioactive liquid effluents are presented in this section.

\subsection{RADIOACTIVE LIQUID EFFLUENTS}

Facilities managed by WHC and facilities monitored by BHI discharge liquid effluents potentially or normally contaminated with radionuclides to the soil or the Columbia River. The general categories of these liquid effluents are: (1) cooling water, (2) steam condensates, (3) process condensates, (4) laboratory and chemical sewer waste water, (5) filter backwash, and (7) groundwater seepage resulting from discharges to the soil.

The measured quantities of radionuclides discharged in liquid effluents to ground disposal facilities and to the Columbia River are summarized in Tables ES-2 and ES-3, respectively. Releases of specific radionuclides and total activity discharged by individual liquid effluent streams is presented in Table 3-1. Included in these tables are releases to the Columbia River adjacent to $100-\mathrm{N}$ Area via groundwater seepage along the stretch of riverbank known as the N-Springs, see Figure 3-1.

Figure 3-1

Historical Liquid Effluent Releases of ${ }^{90} \mathrm{Sr}$ to the Columbia River.

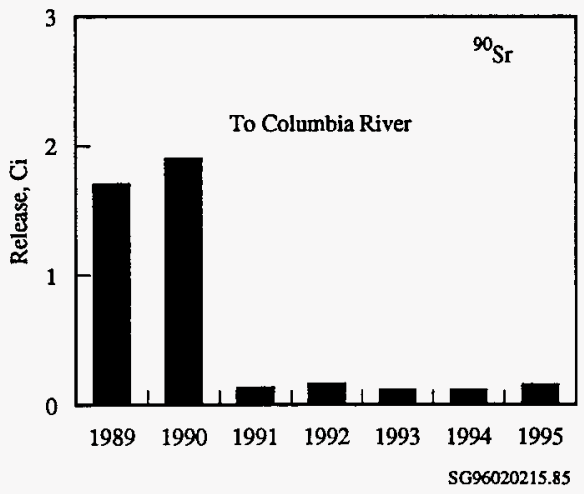


In the $100-\mathrm{N}$ Area, total radionuclide releases continued to be far below past releases during $\mathrm{N}$-Reactor operation.

In the 200 Areas, the 200 East Area's Treated Effluent Disposal Facility (TEDF) started processing liquid effluents from radioactive liquid effluent streams in May of 1995. The 200 Area TEDF's effluents comply with the Clean Water Act and meet the drinking water standard.

The PUREX Plant, which has not operated since March 1990, did not discharge radionuclides in quantities significantly different than were discharged in 1994. The PUREX Plant chemical sewer waste water (CSL), can be diverted either automatically or manually to the 216-A-42 Retention Basin, which prevents the effluents from discharging to the soil. Diversion normally would occur when online radiation or $\mathrm{pH}$ monitors upstream of the diversion valve register potential or real upset conditions in the effluent streams. The 216-A-42 Retention Basin allows sampling and analyzing of diverted suspect effluent while it is temporarily retained there. If analysis demonstrates compliance with release limits, the effluent is discharged to the 216-B-3 Pond. If the effluent is not within applicable release limits, it can be pumped to the PUREX Plant for reprocessing. In May of 1995, the CSL liquid effluent stream started discharging to the 200 Area TEDF. As a result the CSL stream no longer discharges to the environment via $216-\mathrm{B}-3$ pond. The CWL and SCD release points did not discharge liquid effluents in 1995.

The 242-A Evaporator-Crystallizer operated in 1995 to process liquid radioactive material, some effluents were discharged to the 216-B-3 Pond as a result of its operation.

In 1994, two significant upgrades to the PFP Cooling Water Stream (Stream Code 2904-ZA) were completed, affecting the $216-Z-20$ Crib discharge disposal site. First, in January, a closed loop cooling system was placed on-line. This upgrade replaced many of the once through cooling systems with closed loop heat exchangers, significantly reducing effluent discharges to the $216-\mathrm{Z}-20 \mathrm{Crib}$. Many of the effluent sources producing the highest radionuclide releases were also eliminated as a result of this upgrade. The second upgrade was the addition of the 243-Z Low Level Waste Treatment Facility (LLWTF) to the PFP complex, in May of 1994. This facility is designed to remove the remaining radioactive contaminants from the $2904-\mathrm{Z}$ stream. Radioactive contaminants are removed from the liquid effluent stream through the use of treatment canisters equipped with three types of filter media. In May of 1995, the PFP Cooling Water Stream started discharging to the 200 Area TEDF. As a result the stream no longer discharges to the environment via $216-Z-20$ crib.

In May of 1995, the 222-S Laboratory Chemical Sewer (stream code 207-SL) started discharging to the 200 Area TEDF. As a result the 207-SL stream no longer discharges to the environment via $216-S-26$ crib.

Discharges to French drains and from the two sources at $T$ Plant are not sampled or recorded for flow. This is because the effluents are judged not to have a potential of exceeding $4 \%$ of 
the DCG values on a yearly average. Four percent of a DCG is equivalent to 4 mrem, which is the drinking water standard (Radiological Drinking Water Standards: U.S. Environmental Protection Agency, National Primary Drinking Water Regulations [40 CFR 141]; and State of Washington, Rules and Regulations of the State Board of Health Regarding Public Water Systems [WAC 248-54]).

In the $300 \mathrm{Area}$, there were no radioactive liquid effluent releases to the environment in 1995. All radioactive liquid effluents were transported to the 200 Area Tank Farms via the 340 Facility in the 300 Area.

\subsection{NONRADIOACTIVE LIQUID EFFLUENTS}

\subsubsection{Sanitary Sewage Discharges to the Soil}

Various facilities discharged sanitary sewage. In the $100-\mathrm{N}$ Area, sanitary waste water was discharged to the 100-N Sewage Lagoon and five septic tanks. In the 100-B, 100-D, 100-H, and $100-\mathrm{K}$ Areas, sanitary sewage was discharged to septic tanks and drainfields. In the 200 Areas, sanitary waste water was discharged to a system of septic tanks and drainfields. Sludge was pumped from septic tanks in the 200 Areas and taken to the 100-N Sewage Lagoon for disposal. In the 300 Area, sanitary sewage was discharged to the 300 Area septic tank and trench system. In the 400 Area, sanitary sewage was discharged to a septic system.

The estimated volume of sewage discharged by area during 1995 is shown in Table 3-2. All sanitary sewer discharges are estimated by multiplying the total number of employees and subcontractors stationed in each area by $25 \mathrm{gal} /$ day-person.

\subsubsection{National Pollutant Discharge Elimination System Discharges}

Liquid effluents discharged to the Columbia River from the 100-N Area, 100-K Area, and 300 Area are regulated by the parameters in the NPDES permit for the Hanford Site. A list of the discharge points is provided in Table 3-3. A summary of analyses performed to ensure compliance with the NPDES permit is given Table 3-4.

\subsubsection{Process Water Discharges}

Westinghouse Hanford Company managed facilities discharged nonhazardous waste water to the ground at the Hanford Site.

The 200 East Area Water Treatment Facility discharged waste water totalling approximately $6.06 \mathrm{E}+07 \mathrm{~L}(1.60 \mathrm{E}+07 \mathrm{gal})$ to the 216-B-3 Pond. The 200 West Area Water Treatment Facility discharged waste water totalling approximately $4.25 \mathrm{E}+07 \mathrm{~L}(1.12 \mathrm{E}+07$ gal) to percolation ponds, from January to June 1995 . When $284-\mathrm{W}$ discharges to the soil column ceased. 200 Area process water discharges are estimated by multiplying the total minutes in 
a year by their respective average flowrates. Estimated process water discharge flowrates average $150 \mathrm{gal} / \mathrm{min}$ for the 200 East Area and $130 \mathrm{gal} / \mathrm{min}$ for the 200 West Area.

The volume of nonhazardous nonradioactive liquid wastes discharged by the 200 East Area's Treated Effluent Disposal Facility (TEDF) to the soil in 1995 was not provided for this report.

All non-radioactive liquid effluents from the 300 Area are now discharged to the 300 Area's Treated Effluent Disposal Facility (TEDF). The 300 Area's TEDF discharged 3.14 E+08 L (8.29 E+07 gal) of nonhazardous nonradioactive liquid wastes to the Columbia River in 1995.

In the 400 Area, approximately $4.45 \mathrm{E}+07 \mathrm{~L}(1.18 \mathrm{E}+07$ gal) of process nonhazardous nonradioactive waste water from the 400 Area Process Sewer drained to a process pond located north of the 400 Area. Approximately $\leq 1.7 \mathrm{E}+06 \mathrm{~L}$ ( $\leq 4.4 \mathrm{E}+05 \mathrm{gal})$ of liquid wastes (principally storm water) drained to 24 French drains.

Industrial facilities managed by WHC and monitored by BHI did not discharge to the City of Richland publicly owned treatment works (POTW) any waste water that could be classified as a significant industrial discharge. Significant industrial discharges are defined as those meeting any of the following criteria:

- Being subject to national pretreatment standards promulgated under Section 307 (b) or (c) of the Clean Water Act of 1977

- Having any of the priority toxic pollutants listed in 40 CFR 403

- Having toxic pollutants as defined in the Clean Water Act of 1977, Section 307

- Having a discharge flow of $1.5 \mathrm{E}+05 \mathrm{~L}(4.0 \mathrm{E}+04$ gal) or more per average workday

- Having a flow greater than $5 \%$ of the flow of the POTW.

Such discharges are prohibited unless issued a permit by the City of Richland. 
Table 3-1

(5 sheets)

\begin{tabular}{|c|c|c|c|c|c|}
\hline \multicolumn{6}{|c|}{$\begin{array}{l}1995 \text { Radionuclide Liquid Effluent Data } \\
\text { for Individual Effluent Streams Discharged } \\
\text { from WHC and BHI Facilities. }\end{array}$} \\
\hline $\begin{array}{c}\text { Liquid } \\
\text { Effluent Stream" } \\
\text { [Contractor] } \\
\text { (Stream Code, EDP Code) }\end{array}$ & $\begin{array}{c}\text { Discharge } \\
\text { Disposal } \\
\text { Site }\end{array}$ & $\begin{array}{l}\text { Total } \\
\text { Flow } \\
\text { (L) }\end{array}$ & Radionuclide & $\begin{array}{c}\text { Average } \\
\text { Concentration } \\
(\mu \mathrm{Ci} / \mathrm{mL})^{\mathrm{b}}\end{array}$ & $\begin{array}{l}\text { Annual } \\
\text { Release } \\
\text { (Ci) }^{b}\end{array}$ \\
\hline \multicolumn{6}{|c|}{100 Area Discharges to the Columbia River } \\
\hline $\begin{array}{l}\text { N-Springs } \\
\text { [BHI] } \\
(\mathrm{N} / \mathrm{A}, \mathrm{Y} 101)\end{array}$ & Columbia River & $2.0 \mathrm{E}+07$ & $\begin{array}{l}{ }^{3} \mathrm{H} \\
{ }^{60} \mathrm{Co} \\
{ }^{90} \mathrm{Sr} \\
{ }^{106} \mathrm{Ru} \\
{ }^{125} \mathrm{Sb} \\
{ }^{134} \mathrm{Cs} \\
{ }^{137} \mathrm{Cs} \\
{ }^{154} \mathrm{Eu} \\
{ }^{155} \mathrm{Eu} \\
{ }^{238} \mathrm{Pu} \\
{ }^{239.240} \mathrm{Pu} \\
\text { total alpha } \\
\text { total beta }\end{array}$ & $\begin{array}{c}7.4 \mathrm{E}-06 \\
5.7 \mathrm{E}-09 \\
1.0 \mathrm{E}-05 \\
\mathrm{ND} \\
1.4 \mathrm{E}-08 \\
\text { ND } \\
8.0 \mathrm{E}-08 \\
8.8 \mathrm{E}-09 \\
\text { ND } \\
\text { ND } \\
2.2 \mathrm{E}-11 \\
3.0 \mathrm{E}-09 \\
1.1 \mathrm{E}-05\end{array}$ & $\begin{array}{c}1.5 \mathrm{E}-01 \\
1.1 \mathrm{E}-04 \\
2.1 \mathrm{E}-01 \\
\mathrm{ND} \\
2.7 \mathrm{E}-04 \\
\mathrm{ND} \\
1.6 \mathrm{E}-03 \\
1.8 \mathrm{E}-04 \\
\text { ND } \\
\text { ND } \\
4.3 \mathrm{E}-07 \\
5.9 \mathrm{E}-05 \\
2.3 \mathrm{E}-01\end{array}$ \\
\hline $\begin{array}{l}\text { NPDES Outfall 009, } \\
100-\mathrm{N} 102-\text { in. Outfall } \\
{[\mathrm{BHI}]} \\
(\mathrm{N} / \mathrm{A}, \mathrm{Y} 120)\end{array}$ & Columbia River & 0.0 & N/A & N/A & N/A \\
\hline $\begin{array}{l}\text { NPDES Outfall 004, } \\
\text { 100-K } 1908-\mathrm{K} \text { Outfall } \\
\text { [WHC] } \\
(\mathrm{N} / \mathrm{A}, \mathrm{Y} 130)\end{array}$ & Columbia River & $1.6 \mathrm{E}+09$ & $\begin{array}{l}{ }^{3} \mathrm{H} \\
{ }^{60} \mathrm{Co} \\
{ }^{90} \mathrm{Sr} \\
{ }^{106} \mathrm{Ru} \\
{ }^{125} \mathrm{Sb} \\
{ }^{1.44} \mathrm{Cs} \\
{ }^{137} \mathrm{Cs} \\
{ }^{154} \mathrm{Eu} \\
{ }^{153} \mathrm{Eu} \\
{ }^{238} \mathrm{Pu} \\
{ }^{239 / 240} \mathrm{Pu} \\
{ }^{24 !} \mathrm{Am} \\
\text { total alpha } \\
\text { total beta }\end{array}$ & $\begin{array}{c}\text { ND } \\
\text { ND } \\
3.0 \mathrm{E}-10 \\
\text { ND } \\
\text { ND } \\
\text { ND } \\
3.4 \mathrm{E}-10 \\
2.4 \mathrm{E}-09 \\
\text { ND } \\
3.4 \mathrm{E}-12 \\
4.9 \mathrm{E}-13 \\
7.5 \mathrm{E}-12 \\
2.2 \mathrm{E}-10 \\
7.7 \mathrm{E}-09\end{array}$ & $\begin{array}{c}\mathrm{ND} \\
\mathrm{ND} \\
4.9 \mathrm{E}-04 \\
\mathrm{ND} \\
\mathrm{ND} \\
\mathrm{ND} \\
5.6 \mathrm{E}-04 \\
3.9 \mathrm{E}-03 \\
\mathrm{ND} \\
5.5 \mathrm{E}-06 \\
7.9 \mathrm{E}-07 \\
1.2 \mathrm{E}-05 \\
3.5 \mathrm{E}-04 \\
1.2 \mathrm{E}-02\end{array}$ \\
\hline
\end{tabular}


Table 3-1

(5 sheets)

\begin{tabular}{|c|c|c|c|c|c|}
\hline \multicolumn{6}{|c|}{$\begin{array}{l}1995 \text { Radionuclide Liquid Effluent Data } \\
\text { for Individual Effluent Streams Discharged } \\
\text { from WHC and BHI Facilities. }\end{array}$} \\
\hline $\begin{array}{c}\text { Liquid } \\
\text { Effluent Stream }^{\mathrm{a}} \\
\text { [Contractor] }^{\text {(Stream Code, EDP Code) }}\end{array}$ & $\begin{array}{l}\text { Discharge } \\
\text { Disposal } \\
\text { Site }\end{array}$ & $\begin{array}{l}\text { Total } \\
\text { Flow } \\
\text { (L) }\end{array}$ & Radionuclide & $\begin{array}{c}\text { Average } \\
\text { Concentration } \\
(\mu \mathrm{Ci} / \mathrm{mL})^{\mathrm{b}}\end{array}$ & $\begin{array}{c}\text { Annual } \\
\text { Release } \\
(\mathbf{C i})^{\mathrm{b}}\end{array}$ \\
\hline \multicolumn{6}{|c|}{200 Area Discharges to the Ground } \\
\hline $\begin{array}{l}\text { 222-S Laboratory } \\
\text { Chemical Sewer } \\
\text { [WHC] } \\
\text { (207-SL, H101) }\end{array}$ & $216-S-26$ Crib & $2.4 \mathrm{E}+06$ & $\begin{array}{l}{ }^{90} \mathrm{Sr} \\
{ }^{106} \mathrm{Ru} \\
{ }^{125} \mathrm{Sb} \\
{ }^{137} \mathrm{Cs} \\
{ }^{238} \mathrm{Pu} \\
{ }^{239 / 240} \mathrm{Pu} \\
{ }^{241} \mathrm{Am} \\
\text { total alpha } \\
\text { total beta }\end{array}$ & $\begin{array}{c}4.2 \mathrm{E}-09 \\
\mathrm{ND} \\
\mathrm{ND} \\
5.3 \mathrm{E}-09 \\
\mathrm{ND} \\
1.3 \mathrm{E}-10 \\
2.0 \mathrm{E}-10 \\
2.8 \mathrm{E}-10 \\
1.3 \mathrm{E}-09\end{array}$ & $\begin{array}{c}9.9 \mathrm{E}-06 \\
\mathrm{ND} \\
\mathrm{ND} \\
1.2 \mathrm{E}-05 \\
\mathrm{ND} \\
3.0 \mathrm{E}-07 \\
4.7 \mathrm{E}-07 \\
6.6 \mathrm{E}-07 \\
3.1 \mathrm{E}-06\end{array}$ \\
\hline $\begin{array}{l}\text { UO }_{3} \text { Plant Waste } \\
\text { Water } \\
\text { [WHC] } \\
(207-\mathrm{U}, \mathrm{H102})\end{array}$ & 216-U-14 Ditch & 0.0 & $\mathrm{~N} / \mathrm{A}$ & $\mathrm{N} / \mathrm{A}$ & $\mathrm{N} / \mathrm{A}$ \\
\hline $\begin{array}{l}\text { PFP Cooling Water } \\
\text { [WHC] } \\
(2904-\mathrm{ZA}, \mathrm{H} 103)\end{array}$ & 216-Z-20 Crib & $7.3 E+06$ & $\begin{array}{l}{ }^{137} \mathrm{Cs} \\
{ }^{238} \mathrm{Pu} \\
{ }^{239 / 240} \mathrm{Pu} \\
{ }^{241} \mathrm{Am} \\
\text { total alpha } \\
\text { total beta }\end{array}$ & $\begin{array}{l}4.1 \mathrm{E}-09 \\
1.0 \mathrm{E}-09 \\
1.5 \mathrm{E}-08 \\
4.9 \mathrm{E}-09 \\
1.9 \mathrm{E}-08 \\
1.3 \mathrm{E}-09\end{array}$ & $\begin{array}{ll}3.0 & \mathrm{E}-05 \\
7.6 & \mathrm{E}-06 \\
1.1 & \mathrm{E}-04 \\
3.6 & \mathrm{E}-05 \\
1.4 & \mathrm{E}-04 \\
9.6 & \mathrm{E}-06\end{array}$ \\
\hline $\begin{array}{l}\text { 242-A Evaporator } \\
\text { Cooling Water } \\
\text { [WHC] } \\
(\mathrm{ACW}, \mathrm{H} 108)\end{array}$ & 216-B-3 Pond & $7.8 \mathrm{E}+08$ & $\begin{array}{l}{ }^{3} \mathrm{H} \\
{ }^{60} \mathrm{Co} \\
{ }^{90} \mathrm{Sr} \\
{ }^{106} \mathrm{Ru} \\
{ }^{113} \mathrm{Sn} \\
{ }^{137} \mathrm{Cs} \\
\text { Total Uranium } \\
{ }^{238} \mathrm{Pu} \\
{ }^{239 / 240} \mathrm{Pu} \\
{ }^{24 !} \mathrm{Am} \\
\text { total alpha } \\
\text { total beta }\end{array}$ & $\begin{array}{l}\text { ND } \\
\text { ND } \\
N D \\
N D \\
N D \\
N D \\
5.3 \mathrm{E}-10 \\
1.2 \mathrm{E}-11 \\
\mathrm{ND} \\
8.1 \mathrm{E}-11 \\
4.3 \mathrm{E}-10 \\
5.1 \mathrm{E}-10\end{array}$ & $\begin{array}{c}N D \\
N D \\
N D \\
N D \\
N D \\
N D \\
4.2 \mathrm{E}-04 \\
9.7 \mathrm{E}-06 \\
\text { ND } \\
6.3 \mathrm{E}-05 \\
3.4 \mathrm{E}-04 \\
4.0 \mathrm{E}-04\end{array}$ \\
\hline
\end{tabular}


WHC-EP-0527-5

Table 3-1

(5 sheets)

\begin{tabular}{|c|c|c|c|c|c|}
\hline \multicolumn{6}{|c|}{$\begin{array}{l}1995 \text { Radionuclide Liquid Effluent Data } \\
\text { for Individual Effluent Streams Discharged } \\
\text { from WHC and BHI Facilities. }\end{array}$} \\
\hline $\begin{array}{c}\text { Liquid } \\
\text { Effuent Stream }{ }^{\mathrm{a}} \\
\text { [Contractor] }^{\text {[Stream Code, EDP Code) }}\end{array}$ & $\begin{array}{c}\text { Discharge } \\
\text { Disposal } \\
\text { Site }\end{array}$ & $\begin{array}{l}\text { Total } \\
\text { Flow } \\
\text { (L) }\end{array}$ & Radionuclide & $\begin{array}{c}\text { Average } \\
\text { Concentration } \\
(\mu \mathrm{Ci} / \mathrm{mL})^{\mathbf{b}}\end{array}$ & $\begin{array}{c}\text { Annual } \\
\text { Release } \\
(\mathrm{Ci})^{b}\end{array}$ \\
\hline $\begin{array}{l}\text { 242-A Evaporator } \\
\text { Steam Condensate } \\
\text { [WHC] } \\
\text { (ASC, H110) }\end{array}$ & 216-B-3 Pond & $2.3 \mathrm{E}+07$ & $\begin{array}{l}{ }^{3} \mathrm{H} \\
{ }^{60} \mathrm{Co} \\
{ }^{90} \mathrm{Sr} \\
{ }^{106} \mathrm{Ru} \\
{ }^{113} \mathrm{Sn} \\
{ }^{137} \mathrm{Cs} \\
{ }^{238} \mathrm{Pu} \\
{ }^{239} 240 \mathrm{Pu} \\
{ }^{241} \mathrm{Am} \\
\text { total alpha } \\
\text { total beta }\end{array}$ & $\begin{array}{c}6.6 \mathrm{E}-07 \\
\mathrm{ND} \\
\mathrm{ND} \\
\mathrm{ND} \\
\mathrm{ND} \\
\mathrm{ND} \\
9.6 \mathrm{E}-11 \\
7.6 \mathrm{E}-11 \\
8.7 \mathrm{E}-11 \\
8.8 \mathrm{E}-11 \\
3.5 \mathrm{E}-10\end{array}$ & $\begin{array}{c}1.5 \mathrm{E}-02 \\
\mathrm{ND} \\
\mathrm{ND} \\
\mathrm{ND} \\
\mathrm{ND} \\
\mathrm{ND} \\
2.2 \mathrm{E}-06 \\
1.7 \mathrm{E}-06 \\
2.0 \mathrm{E}-06 \\
2.0 \mathrm{E}-06 \\
7.9 \mathrm{E}-06\end{array}$ \\
\hline $\begin{array}{l}\text { 241-A Tank Farm } \\
\text { Cooling Water } \\
\text { [WHC] } \\
\text { (CA8, H115) }\end{array}$ & 216-B-3 Pond & $8.3 E+08$ & $\begin{array}{l}{ }^{60} \mathrm{Co} \\
{ }^{90} \mathrm{Sr} \\
{ }^{106} \mathrm{Ru} \\
{ }^{113} \mathrm{Sn} \\
{ }^{125} \mathrm{Sb} \\
{ }^{134} \mathrm{Cs} \\
3^{137} \mathrm{Cs} \\
{ }^{238} \mathrm{Pu} \\
{ }^{239 / 240} \mathrm{Pu} \\
{ }^{241} \mathrm{Am} \\
\text { total alpha } \\
\text { total beta }\end{array}$ & $\begin{array}{c}2.0 \mathrm{E}-09 \\
\text { ND } \\
4.1 \mathrm{E}-08 \\
1.0 \mathrm{E}-09 \\
\text { ND } \\
1.1 \mathrm{E}-09 \\
1.5 \mathrm{E}-09 \\
9.6 \mathrm{E}-12 \\
2.2 \mathrm{E}-11 \\
1.0 \mathrm{E}-10 \\
4.0 \mathrm{E}-10 \\
3.6 \mathrm{E}-10\end{array}$ & $\begin{array}{c}1.7 \mathrm{E}-03 \\
\mathrm{ND} \\
3.4 \mathrm{E}-02 \\
8.5 \mathrm{E}-04 \\
\mathrm{ND} \\
9.0 \mathrm{E}-04 \\
1.2 \mathrm{E}-03 \\
8.0 \mathrm{E}-06 \\
1.8 \mathrm{E}-05 \\
8.6 \mathrm{E}-05 \\
3.3 \mathrm{E}-04 \\
3.0 \mathrm{E}-04\end{array}$ \\
\hline $\begin{array}{l}\text { 244-AR Vault } \\
\text { Cooling Water } \\
\text { [WHC] } \\
\text { (CAR, H116) }\end{array}$ & 216-B-3 Pond & $4.8 \mathrm{E}+06$ & $\begin{array}{l}{ }^{60} \mathrm{Co} \\
{ }^{90} \mathrm{Sr} \\
{ }^{106} \mathrm{Ru} \\
{ }^{113} \mathrm{Sn} \\
{ }^{125} \mathrm{Sb} \\
{ }^{134} \mathrm{Cs} \\
{ }^{137} \mathrm{Cs} \\
{ }^{238} \mathrm{Pu} \\
{ }^{239 / 240} \mathrm{Pu} \\
{ }^{241} \mathrm{Am} \\
\text { total alpha } \\
\text { total beta }\end{array}$ & 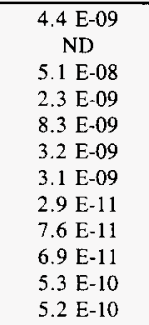 & $\begin{array}{cc}2.1 & \mathrm{E}-05 \\
& \mathrm{ND} \\
2.4 & \mathrm{E}-04 \\
1.1 & \mathrm{E}-05 \\
4.0 & \mathrm{E}-05 \\
1.6 & \mathrm{E}-05 \\
1.5 & \mathrm{E}-05 \\
1.4 & \mathrm{E}-07 \\
3.7 & \mathrm{E}-07 \\
3.3 & \mathrm{E}-07 \\
2.5 & \mathrm{E}-06 \\
2.5 & \mathrm{E}-06\end{array}$ \\
\hline
\end{tabular}


Table 3-1

(5 sheets)

\section{Radionuclide Liquid Effluent Data \\ for Individual Effluent Streams Discharged from WHC and BHI Facilities.}

\begin{tabular}{|c|c|c|c|c|c|}
\hline $\begin{array}{c}\text { Liquid } \\
\text { Effluent Stream" } \\
\text { [Contractor] } \\
\text { (Stream Code, EDP Code) }\end{array}$ & $\begin{array}{l}\text { Discharge } \\
\text { Disposal } \\
\text { Site }\end{array}$ & $\begin{array}{l}\text { Total } \\
\text { Flow } \\
\text { (L) }\end{array}$ & Radionuclide & $\begin{array}{c}\text { Average } \\
\text { Concentration } \\
(\mu \mathrm{Ci} / \mathrm{mL})^{\mathrm{b}}\end{array}$ & $\begin{array}{l}\text { Annual } \\
\text { Release } \\
\text { (Ci) }^{\mathbf{b}}\end{array}$ \\
\hline $\begin{array}{l}\text { B-Plant Cooling Water } \\
\text { [WHC] } \\
\text { (CBC, H117) }\end{array}$ & 216-B-3 Pond & $3.0 \mathrm{E}+09$ & $\begin{array}{l}{ }^{60} \mathrm{Co} \\
{ }^{90} \mathrm{Sr} \\
{ }^{106} \mathrm{Ru} \\
{ }^{113} \mathrm{Sn} \\
{ }^{125} \mathrm{Sb} \\
{ }^{134} \mathrm{Cs} \\
{ }^{137} \mathrm{Cs} \\
\text { total alpha } \\
\text { total beta }\end{array}$ & $\begin{array}{c}2.5 \mathrm{E}-09 \\
3.8 \mathrm{E}-10 \\
5.2 \mathrm{E}-08 \\
3.1 \mathrm{E}-09 \\
1.0 \mathrm{E}-09 \\
\mathrm{ND} \\
1.7 \mathrm{E}-09 \\
3.6 \mathrm{E}-10 \\
1.3 \mathrm{E}-09\end{array}$ & $\begin{array}{c}7.4 \mathrm{E}-03 \\
1.1 \mathrm{E}-03 \\
1.6 \mathrm{E}-01 \\
9.3 \mathrm{E}-03 \\
3.1 \mathrm{E}-03 \\
\mathrm{ND} \\
5.1 \mathrm{E}-03 \\
1.1 \mathrm{E}-03 \\
3.8 \mathrm{E}-03\end{array}$ \\
\hline $\begin{array}{l}\text { PUREX Chemical Sewer } \\
\text { [WHC] } \\
\text { (CSL, H118) }\end{array}$ & 216-B-3 Pond & $1.8 \mathrm{E}+08$ & $\begin{array}{l}{ }^{3} \mathrm{H} \\
{ }_{90}^{90} \mathrm{Sr} \\
{ }^{99} \mathrm{Tc} \\
{ }^{106} \mathrm{Ru} \\
{ }^{113} \mathrm{Sn} \\
{ }^{137} \mathrm{Cs} \\
\text { Total Uranium } \\
{ }^{238} \mathrm{Pu} \\
{ }^{239 / 240} \mathrm{Pu} \\
{ }^{241} \mathrm{Am} \\
\text { total alpha } \\
\text { total beta }\end{array}$ & $\begin{array}{l}\mathrm{ND} \\
3.4 \mathrm{E}-09 \\
1.2 \mathrm{E}-09 \\
\mathrm{ND} \\
\mathrm{ND} \\
\mathrm{ND} \\
2.7 \mathrm{E}-10 \\
1.4 \mathrm{E}-10 \\
2.8 \mathrm{E}-09 \\
4.9 \mathrm{E}-10 \\
1.7 \mathrm{E}-10 \\
5.9 \mathrm{E}-10\end{array}$ & $\begin{array}{c}\text { ND } \\
6.3 \mathrm{E}-04 \\
2.3 \mathrm{E}-04 \\
\text { ND } \\
\text { ND } \\
\text { ND } \\
5.1 \mathrm{E}-05 \\
2.6 \mathrm{E}-05 \\
5.1 \mathrm{E}-04 \\
9.1 \mathrm{E}-05 \\
3.2 \mathrm{E}-05 \\
1.1 \mathrm{E}-04\end{array}$ \\
\hline $\begin{array}{l}\text { 242-S Evaporator } \\
\text { Steam Condensate } \\
\text { [WHC] } \\
\text { (RC-1, H122) }\end{array}$ & 216-U-14 Ditch & 0.0 & $\mathrm{~N} / \mathrm{A}$ & N/A & $\mathrm{N} / \mathrm{A}$ \\
\hline $\begin{array}{l}\text { UO, Plant Process } \\
\text { Condensate } \\
\text { [WHC] } \\
\text { (U-17, H126) }\end{array}$ & 216-U-17 Crib & 0.0 & $\mathrm{~N} / \mathrm{A}$ & N/A & N/A \\
\hline
\end{tabular}


Table 3-1

\section{Radionuclide Liquid Effluent Data \\ for Individual Effluent Streams Discharged from WHC and BHI Facilities.}

\begin{tabular}{|c|c|c|c|c|c|}
\hline $\begin{array}{c}\text { Liquid } \\
\text { Effluent Stream }{ }^{2} \\
\text { [Contractor] } \\
\text { (Stream Code, EDP Code) }\end{array}$ & $\begin{array}{c}\text { Discharge } \\
\text { Disposal } \\
\text { Site }\end{array}$ & $\begin{array}{c}\text { Total } \\
\text { Flow } \\
\text { (L) }\end{array}$ & Radionuclide & $\begin{array}{c}\text { Average } \\
\text { Concentration } \\
(\mu \mathrm{Ci} / \mathrm{mL})^{\mathrm{b}}\end{array}$ & $\begin{array}{c}\text { Annual } \\
\text { Release } \\
(\mathbf{C i})^{\mathbf{b}}\end{array}$ \\
\hline \multicolumn{6}{|c|}{300 Area Discharges to the Ground } \\
\hline $\begin{array}{l}300 \text { Area Process Sewer } \\
\text { [WHC] }\end{array}$ & 316-5 Trench & 0.0 & N/A & $\mathrm{N} / \mathrm{A}$ & N/A \\
\hline
\end{tabular}

\section{Notes:}

a WHC $=$ Westinghouse Hanford Company; $\mathrm{BHI}=$ Bechtel Hanford, Inc; EDP Code = Electronic Data Processing Code.

b $1 \mu \mathrm{Ci} / \mathrm{mL}=3.7 \mathrm{E}+10 \mathrm{bq} / \mathrm{m}^{3} ; 1$ Curie $=3.7 \mathrm{E}+10$ becquerel; $\mathrm{ND}=$ None Detected.

c PUREX Chemical Sewer releases include all discharges to PUREX A-42 Diversion Basin. 
WHC-EP-0527-5

Table 3-2

\begin{tabular}{|c|c|c|}
\hline \multicolumn{3}{|c|}{ Sanitary Sewage Discharged in 1995 } \\
\hline \multicolumn{2}{|c|}{ Discharge $^{2}$} \\
Area & (L/yr) & (gal/yr) \\
\hline $100-\mathrm{D}$ & $1.4 \mathrm{E}+05$ & $3.7 \mathrm{E}+04$ \\
$100-\mathrm{H}$ & $1.4 \mathrm{E}+05$ & $3.7 \mathrm{E}+04$ \\
$100-\mathrm{K}$ & $1.1 \mathrm{E}+07$ & $2.9 \mathrm{E}+06$ \\
$100-\mathrm{N}$ & $1.2 \mathrm{E}+07$ & $3.3 \mathrm{E}+06$ \\
$200 \mathrm{East}$ & $1.1 \mathrm{E}+08$ & $3.0 \mathrm{E}+07$ \\
$200 \mathrm{West}$ & $6.8 \mathrm{E}+07$ & $1.8 \mathrm{E}+07$ \\
300 & $7.2 \mathrm{E}+07$ & $1.9 \mathrm{E}+07$ \\
400 & $1.8 \mathrm{E}+07$ & $4.8 \mathrm{E}+06$ \\
\hline
\end{tabular}

a Discharges estimated by multiplying the total number of persons assigned to each area by 25 gal./day-person. 
WHC-EP-0527-5

Table 3-3

\begin{tabular}{|c|c|}
\hline \multicolumn{2}{|c|}{$\begin{array}{c}\text { National Pollutant Discharge Elimination System } \\
\text { (NPDES) Discharge Points }\end{array}$} \\
\hline Designation & \multicolumn{1}{c|}{ Description } \\
\hline $001 \mathrm{~A}$ & 300 Area Treated Effluent Disposal Facility (TEDF) \\
\hline $003^{*}$ & $181-\mathrm{KE}$ Inlet Screen Backwash \\
\hline 004 & $1908-\mathrm{K}$ Outfall \\
\hline $005^{*}$ & $182-\mathrm{N}$ Tank Farm Overflow (36-in. raw water return) \\
\hline $006^{*}$ & $182-\mathrm{N}$ Drain System (42-in. raw water return) \\
\hline $007^{*}$ & $181-\mathrm{N}$ Inlet Screen Backwash \\
\hline $009^{*}$ & 102 -in. Outfall (raw water return) \\
\hline N-Springs & $100-\mathrm{N}$ Riverbank Springs \\
\hline & \\
\hline
\end{tabular}

* Did not discharge any effluent during 1995. 
Table 3-4

(2 sheets)

\begin{tabular}{|c|c|c|c|c|c|c|}
\hline \multicolumn{7}{|c|}{$\begin{array}{l}\text { Summary of National Pollutant Discharge System } \\
\text { (NPDES) Data System for } 1995^{\circ}\end{array}$} \\
\hline \multirow[t]{2}{*}{ Sample Parameter } & \multicolumn{2}{|c|}{$\begin{array}{c}\text { 1908-K Outfall } \\
(004)\end{array}$} & \multicolumn{2}{|c|}{$\begin{array}{c}\text { N-Springs } \\
\text { (1301-N) }\end{array}$} & \multicolumn{2}{|c|}{$\begin{array}{c}300 \text { Area TEDF } \\
(001 \mathrm{~A})\end{array}$} \\
\hline & Avg & $\operatorname{Max}$ & Avg & Max & Avg & $\operatorname{Max}$ \\
\hline Flowrate (MGD) & 1.179 & 3.860 & $*$ & $*$ & 0.251 & 0.450 \\
\hline Temperature $\left({ }^{\circ} \mathrm{F}\right)$ & $*$ & 75 & $*$ & 66 & 81 & 95 \\
\hline pH & 7.6 & 7.9 & 7.1 & 7.5 & 6.6 & 8.9 \\
\hline Total Suspended Solids ( $\mu \mathrm{g} / \mathrm{L})$ & 1000 & 4000 & $*$ & $*$ & 738 & 11000 \\
\hline Oil and Grease $(\mathrm{mg} / \mathrm{L})$ & $*$ & $*$ & 4 & 5 & $*$ & * \\
\hline Aluminum $(\mu \mathrm{g} / \mathrm{L})$ & * & $*$ & $*$ & * & 38.0 & 144.0 \\
\hline Arsenic $(\mu \mathrm{g} / \mathrm{L})$ & * & * & $*$ & $*$ & 0.5 & 3.0 \\
\hline Beryllium $(\mu \mathrm{g} / \mathrm{L})$ & $*$ & * & * & * & 0.06 & 1.50 \\
\hline Cadmium $(\mu \mathrm{g} / \mathrm{L})$ & * & * & * & $*$ & 5.9 & 105.0 \\
\hline Chromium (mg/L) & $*$ & * & 0.004 & 0.004 & * & * \\
\hline Chlorine (mg/L) & $<0.01$ & 0.03 & * & * & $*$ & $*$ \\
\hline Copper $(\mu \mathrm{g} / \mathrm{L})$ & * & * & $*$ & $*$ & 2 & 6 \\
\hline $\operatorname{Iron}(\mu \mathrm{g} / \mathrm{L})$ & * & * & 300 & 700 & 18.4 & 75.1 \\
\hline Lead $(\mu \mathrm{g} / \mathrm{L})$ & $*$ & $*$ & * & $*$ & 0.2 & 2.3 \\
\hline Manganese $(\mu \mathrm{g} / \mathrm{L})$ & * & * & * & * & 0.2 & 1.5 \\
\hline Mercury $(\mu \mathrm{g} / \mathrm{L})$ & $*$ & $*$ & $*$ & * & $<0.1$ & 0.2 \\
\hline Nickel $(\mu \mathrm{g} / \mathrm{L})$ & * & * & * & $*$ & 0.7 & 4.8 \\
\hline Radium-226 (pCi/L) & $*$ & * & * & $*$ & 0.008 & 0.100 \\
\hline Radium-228 (pCi/L) & $*$ & $*$ & * & * & 0.066 & 0.900 \\
\hline Selenium $(\mu \mathrm{g} / \mathrm{L})$ & * & * & $*$ & * & $<3$ & $<3$ \\
\hline Silver $(\mu \mathrm{g} / \mathrm{L})$ & * & * & * & 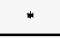 & 0.4 & 2.6 \\
\hline Zinc $(\mu \mathrm{g} / \mathrm{L})$ & * & * & $*$ & * & 8.8 & 52.0 \\
\hline Ammonia (as nitrogen) $(\mu \mathrm{g} / \mathrm{L})$ & $*$ & * & 50 & 50 & 123 & 578 \\
\hline $\begin{array}{l}\text { Bis (2-ethylhexyl) phthalate } \\
(\mu \mathrm{g} / \mathrm{L})\end{array}$ & * & * & $*$ & * & $<3$ & $<3$ \\
\hline Chlorodiflouromethane $(\mu \mathrm{g} / \mathrm{L})$ & $*$ & * & * & $*$ & $<0.1$ & $<0.1$ \\
\hline Chloroform $(\mu \mathrm{g} / \mathrm{L})$ & * & * & * & * & 3 & 15 \\
\hline Coliform (growth/100mL) & * & * & 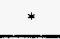 & $*$ & $<3.70$ & $<3.70$ \\
\hline
\end{tabular}


Table 3-4

(2 sheets)

\begin{tabular}{|c|c|c|c|c|c|c|}
\hline \multicolumn{7}{|c|}{$\begin{array}{l}\text { Summary of National Pollutant Discharge System } \\
\text { (NPDES) Data System for } 1995^{\mathrm{a}}\end{array}$} \\
\hline \multirow[t]{2}{*}{ Sample Parameter } & \multicolumn{2}{|c|}{$\begin{array}{c}\text { 1908-K Outfall } \\
\text { (004) }\end{array}$} & \multicolumn{2}{|c|}{$\begin{array}{c}\text { N-Springs } \\
(1301-N)\end{array}$} & \multicolumn{2}{|c|}{$\begin{array}{c}300 \text { Area TEDF } \\
\text { (001A) }\end{array}$} \\
\hline & Avg & $\mathbf{M a x}$ & Avg & Max & Avg & $\operatorname{Max}$ \\
\hline Cyanide $(\mu \mathrm{g} / \mathrm{L})$ & * & * & * & * & 1 & 14 \\
\hline Dichlorobromomethane $(\mu \mathrm{g} / \mathrm{L})$ & $*$ & * & * & * & $<2.2$ & $<2.2$ \\
\hline 1,1-Dichloroethane $(\mu \mathrm{g} / \mathrm{L})$ & * & * & * & $*$ & $<4.7$ & $<4.7$ \\
\hline Methylene Chloride $(\mu \mathrm{g} / \mathrm{L})$ & * & $*$ & $*$ & * & $<3$ & 7 \\
\hline Nitrite $\left(\mathrm{NO}_{2}\right)(\mu \mathrm{g} / \mathrm{L})$ & $*$ & * & * & * & $<60$ & $<60$ \\
\hline Tetrachloroethylene $(\mu \mathrm{g} / \mathrm{L})$ & $*$ & $*$ & * & $*$ & $<5$ & 6 \\
\hline 1.1.1-Trichloroethane $(\mu \mathrm{g} / \mathrm{L})$ & * & * & * & * & $<5.00$ & $<5.00$ \\
\hline Trichloroethylene $(\mu \mathrm{g} / \mathrm{L})$ & $*$ & $*$ & $*$ & $*$ & $<1.9$ & $<1.9$ \\
\hline Toluene $(\mu \mathrm{g} / \mathrm{L})$ & * & * & * & * & 1 & 12 \\
\hline
\end{tabular}

a $\mathrm{MGD}=$ million gallons per day: ${ }^{*}=$ analysis not required. 
THIS PAGF TNTENTIONALLY LEFT BLANK

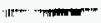


WHC-EP-0527-5

\subsection{HAZARDOUS SUBSTANCE RELEASES}

A hazardous substance release requires notification to appropriate federal, state, and local authorities when established limits are exceeded. Reportable releases of hazardous substances are classified as the following two types:

- Nonroutine releases

- Continuous, routine releases.

Information for each type of release is discussed separately below.

\subsection{NONROUTINE RELEASES}

The following listing shows the number of nonroutine releases that occurred in 1995 , distinguished by the nature of the notifications. Other nonroutine releases and spills are not discussed in this section because they were less than agency reportable limits and the levels in DOE reporting criteria (EPA 1990b, DOE 1993b).

- 13 releases ( $12 \mathrm{WHC}$ and WHC contractors, $1 \mathrm{BHI}$ and BHI contractors) were reported to a regulatory agency

- 430 non-reportable releases (368 WHC and WHC contractors, $62 \mathrm{BHI}$ and BHI contractors) were recorded

\subsection{ROUTINE CONTINUOUS RELEASES}

Releases of hazardous substances that exceed CERCLA reportable quantities (RQ) need not be reported immediately to the National Response Center when both of the following conditions are met:

- An initial notification has been completed

- The routine releases are continuous and stable in quantity and rate.

The initial notification requirement has been satisfied concerning hazardous substances that have exceeded or have a potential to exceed an RQ. Historically only the continuous routine releases of ammonia, ammonium hydroxide, and carbon tetrachloride have posed operational difficulties in staying beneath RQs. For 1995, releases of ammonia, ammonium hydroxide, and carbon tetrachloride were below reportable quantities. Information on the routine releases of hazardous substances are presented in Table 4-1. 
Table 4-1

Comprehensive Environmental Response, Compensation, and Liability Act of 1980 Continuous Release Summary for Calendar Year 1995."

\begin{tabular}{|c|c|c|c|c|c|}
\hline \multicolumn{6}{|c|}{$\begin{array}{c}\text { Comprehensive Environmental Response, Compensation, and Liability Act of } 1980 \\
\text { Continuous Release Summary for Calendar Year 1995." }\end{array}$} \\
\hline Release Point & Substance & $\begin{array}{c}\text { CERCLA } \\
\text { RQ }^{b} \\
(\mathrm{~kg}[\mathrm{bb})\end{array}$ & $\begin{array}{l}\text { Number of } \\
\text { Occurrences } \\
\text { Exceeding } \\
\text { RQ Value }\end{array}$ & $\begin{array}{l}\text { Mean } \\
\text { Release } \\
\text { (kg [lb]) }\end{array}$ & $\begin{array}{l}\text { Annual } \\
\text { Quantity } \\
\text { (kg [lb]) }\end{array}$ \\
\hline $\begin{array}{l}\text { 296-A-24 (PUREX Plant Ammonia } \\
\text { scrubber waste concentrator } \\
\text { exhaust) }\end{array}$ & ammonia & $\begin{array}{c}45.4 \\
(100.0)\end{array}$ & 0 & \multicolumn{2}{|c|}{ (did not operate) } \\
\hline $\begin{array}{l}200 \text { East Area Tank Farms and } \\
\text { 242-A Evaporator }\end{array}$ & ammonia & $\begin{array}{c}45.4 \\
(100.0)\end{array}$ & 0 & $\begin{array}{c}16.9 \\
{[37.3]}\end{array}$ & $\begin{array}{c}6.18 \mathrm{E}+03 \\
{[1.36 \mathrm{E}+04]}\end{array}$ \\
\hline 200 West Area Tank Farms & ammonia & $\begin{array}{c}45.4 \\
(100.0)\end{array}$ & 0 & $\begin{array}{c}4.2 \\
{[9.2]}\end{array}$ & $\begin{array}{r}1.53 \mathrm{E}+03 \\
{[3.37 \mathrm{E}+03]}\end{array}$ \\
\hline $\begin{array}{l}200 \text { West Area } \mathrm{CCl}_{4} \text { Vapor } \\
\text { Extraction Project }\end{array}$ & $\begin{array}{l}\text { carbon } \\
\text { tetrachloride }\end{array}$ & $\begin{array}{c}4.54 \\
(10.00)\end{array}$ & 0 & $\begin{array}{c}0.25 \\
{[0.55]}\end{array}$ & $\begin{array}{c}9.07 \mathrm{E}+01 \\
{[2.00 \mathrm{E}+02\}}\end{array}$ \\
\hline $\begin{array}{l}291-Z-1 \text { (Plutonium Reclamation } \\
\text { Facility) }\end{array}$ & $\begin{array}{l}\text { carbon } \\
\text { tetrachloride }\end{array}$ & $\begin{array}{c}4.54 \\
(10.00)\end{array}$ & 0 & \multicolumn{2}{|c|}{ (did not operate) } \\
\hline
\end{tabular}

a All quantities are for any continuous 24 -hr period.

b CERCLA = Comprehensive Environmental Response, Compensation, and Liability Act of 1980; RQ = Reportable quantity

c Mean release is the annual quantity divided by the number of operating days in the calendar year (e.g. 365 days)

d $\mathrm{NA}=$ Not Available. 


\subsection{REFERENCES}

DOE, 1990a, Environmental Protection. Safety, and Health Protection Information Reporting Requirements, DOE Order 5484.1, U.S. Department of Energy, Washington, D.C.

DOE, 1990b, General Environmental Protection Program, DOE Order 5400.1, U.S. Department of Energy, Washington, D.C.

DOE, 1993a, Radiation Protection of the Public and the Environment, DOE Order 5400.5, U.S. Department of Energy, Washington, D.C.

DOE, 1993b, Environment, Safety, and Health Program for Department of Energy Operations, DOE Order 5480.1B, U.S. Department of Energy, Washington, D.C.

DOE, 1991, Environmental Regulatory Guide for Radiological Effluent Monitoring and Environmental Surveillance, DOE/EH-0173T, U.S. Department of Energy, Washington D.C.

DOE-RL, 1994, Radionuclide Air Emissions Report for the Hanford Site, Calendar Year 1993, DOE/RL-94-51, U.S. Department of Energy, Richland Field Office, Richland, Washington.

DOE-RL, 1995, Radionuclide Air Emissions Report for the Hanford Site, Calendar Year 1994, DOE/RL-95-49, U.S. Department of Energy, Richland Field Office, Richland, Washington.

DOE-RL, 1996, Radionuclide Air Emissions Repon for the Hanford Site, Calendar Year 1995, DOE/RL-96-37, U.S. Department of Energy, Richland Field Office, Richland, Washington.

EPA, 1989, National Emission Standards for Hazardous Air Pollutants, Title 40 Code of Federal Regulations, Part 61, Subpart H, U.S. Environmental Protection Agency, Washington, D.C.

EPA, 1990a, AIRS Facility Subsystem Source Classification Codes and Emission Factor Listing Criteria Air Pollutants, EPA 450/4-90-003, U.S. Environmental Protection Agency, Research Triangle Park, North Carolina.

EPA, 1990b, Designation, reportable Quantities, and Notification, Title 40 Code of Federal Regulations, Part 302, U.S. Environmental Protection Agency, Washington D.C.

PNNL, 1996, Hanford Site Environmental Report for Calendar Year 1995, PNNL-11139, Pacific Northwest National Laboratory, Richland, Washington. 
WHC-EP-0527-5

This page intentionally left blank. 
WHC-EP-0527-5

APPENDIX A

POINT SOURCES FOR RADIONUCLIDE AIR EMISSIONS 
TH: FAT INTENTIONALLY LEI BLANK

1 
WHC-EP-0527-5

\section{APPENDIX A}

\section{POINT SOURCES FOR RADIONUCLIDE AIR EMISSIONS}

\section{A.1 RADIONUCLIDE AIR EMISSIONS SOURCES}

Radionuclide air emissions generally are discharged from point sources (e.g. stacks and vents). Sizes, shapes, and discharge paths of point sources vary depending on facility requirements at the time of the construction. Point source discharge heights range from a few meters to $61 \mathrm{~m}(200 \mathrm{ft})$, and flow rates range from less than $2,832 \mathrm{~L} / \mathrm{min}\left(100 \mathrm{ft}^{3} / \mathrm{min}\right)$ to more than 5.7 million $\mathrm{L} / \mathrm{min}\left(200,000 \mathrm{ft}^{3} / \mathrm{min}\right)$.

The following methods are used to remove radionuclides from the point source emissions: (1) high-efficiency particulate air (HEPA) filters, (2) charcoal absorbers, (3) sand filters, (4) water scrubbers, (5) fiberglass prefilters, and (6) deep-bed fiberglass filters. Generally, at least one stage of HEPA filtration is used as the final particulate emission removal method before emissions are discharged to the atmosphere. All filters are required to have an efficiency of $99.95 \%$ for removing airborne particles larger than $0.3 \mu \mathrm{m}$ in diameter. Filter efficiency is routinely tested.

\section{A.2 POINT SOURCE DESCRIPTIONS}

\section{A.2.1 General Description and Reporting Criteria}

Radionuclide air emissions from point sources are generally are discharged from stacks and vents. Point source sizes, shapes, and discharge paths vary because of facility requirements at the time of construction. Discharge heights range from a few feet to $200 \mathrm{ft}$ $(61 \mathrm{~m})$, and flow rates range from less than $100 \mathrm{ft}^{3} / \mathrm{min}(2,832 \mathrm{~L} / \mathrm{min})$ to more than $200,000 \mathrm{ft}^{3} / \mathrm{min}(5.7 \mathrm{million} \mathrm{L} / \mathrm{min})$. Point sources vary in design from horizontal to vertical, rectangular to cylindrical, and permanent to portable.

The following methods are used singly or in combination to remove radionuclides from most point source emissions: (1) high-efficiency particulate air (HEPA) filters; (2) sand filters; (3) deep-bed fiberglass filters; (4) fibergiass prefilters; (5) charcoal absorbers; and (6) water scrubbers. Generally, between one and three stages of HEPA filtration are used as the final particle removal method before an emission is exhausted to the atmosphere.

Point source emissions are reported in this document when the following criteria were met during 1995: (1) point source required continuous monitoring or periodic confirmatory measurements by 40 CFR 61 Subpart H or WAC 246-247, (2) point source was registered with Washington State Department of Health (WDOH), (3) the point source normally had radionuclide emissions or potentially had radionuclide emissions. Point sources not included 
in this report did not meet the previous criteria or were rendered inoperable (e.g. sealed off) before 1995, as indicated by the previous year's annual report.

\section{A.2.2 100 Areas Facilities}

The 100 Areas contain eight inactive production reactors, the $\mathrm{N}$ Reactor (now in transition to deactivation and decommissioning), and associated support facilities.

Radionuclide air emission points at facilities in the 100-N Area and 100-K Area are described briefly below and shown respectively in Figures 1-2 and 1-3.

A.2.2.1 N Reactor. This dual-purpose reactor operated until 1987, producing electrical power for the regional energy system and plutonium for weapons production. The reactor and associated facilities are currently being deactivated prior to decommissioning.

- 116-N. This stack exhausts filtered air from the 105-N Reactor Building. Emission monitoring consists of a record sampler. In $1993 \mathrm{~N}$ Reactor ventilation was reconfigured such that the $105-\mathrm{N}$ Basin Transfer Area emissions exhaust through the $116-\mathrm{N}$ stack.

- 109-N-Z-I. When this vent is operational, unfiltered air was released through it from 109-N Steam Generator Cells 1 through 5. Emission monitoring consisted of a record sampler and a gross-gamma-energy monitor. This vent did not operate in 1995 and was deregistered in 1995.

- 109-N-C-6. When this vent is operational, unfiltered air is released through it from Steam Generator Cell 6 in the 109-N Building. Emission monitoring consisted of a record sampler and a gross-gamma-energy monitor. This vent did not operate in 1995 and was deregistered in 1995.

- 107-N. This vent exhausts filtered air from the 107-N Basin Recirculation Building. Emissions monitoring consists of a record sampler.

- 105-N 14-Ft. Decontamination Room. This stack exhausts filtered air from a small room in which radioactively contaminated tools are decontaminated. Emissions are continuously monitored by a record sampler.

A.2.2.2 100-K East and West Areas. These areas contain two retired reactors awaiting decommissioning, two water-filled storage basins storing irradiated nuclear fuel, and radiological analysis laboratories.

- 105-KE. This point source consists of four powered vents exhausting unfiltered air from the spent fuel storage basin in the 105-KE Building. Emission monitoring consists of three record samplers. 
- 105-KW. This point source consists of four powered vents exhausting unfiltered air from the spent fuel storage basin in the 105-KW Building. Emission monitoring consists of three record samplers.

- 1706-KE. This stack exhausts filtered air from the 1706-KE Laboratory. Emission monitoring consists of a record sampler.

- 1706-KER. This stack exhausts filtered air from the basement area (approximately $8.2 \mathrm{~m}$ [ 27 feet] below grade) of the 1706-KE Laboratory. Emission monitoring consists of a record sampler.

\section{A.2.3 200 East and West Area Facilities}

The 200 Areas contain facilities for chemical separations, processing, and waste handling and disposal. The radionuclide air emission discharge points in the 200 East Area are shown in Figure 1-4 and those in the 200 West Area, in Figure 1-5. The PUREX Plant and related tank farm and evaporator facilities discharge volatile forms of radionuclides, specifically ${ }^{3} \mathrm{H},{ }^{106} \mathrm{Ru},{ }^{125} \mathrm{Sb}$, and ${ }^{129} \mathrm{I}$. When operating, the PUREX Plant discharged ${ }^{85} \mathrm{Kr}$, but it has not operated fully since December 1989. No activities conducted in the 200 Areas in 1995 resulted in a discharge of ${ }^{85} \mathrm{Kr}$.

A.2.3.1 PUREX Plant. The PUREX Plant is a nuclear-fuel reprocessing plant constructed in 1956 and operated until 1972, when it was placed on standby status until November of 1983. In November of 1983 the PUREX Plant resumed operations, processing a backlog of irradiated fuel from $\mathrm{N}$ Reactor. The plant was again placed in standby following a stabilization run that ended in March 1990. Shutdown orders were issued to PUREX in December 1992, and as a result, the plant is being deactivated.

- 291-A-1. This stack exhausts filtered air from canyon ventilation (Cells A to $\mathrm{M})$, and vessel and condenser vents. Emission monitoring consists of a record sampler, a silver zeolite cartridge, an alpha continuous air monitor (CAM), and a beta-gamma CAM.

- 296-A-1. This stack exhausts filtered air from N and Q Cells, Product Removal rooms, and gloveboxes in these rooms. Emission monitoring consists of a record sampler and an alpha CAM.

- 296-A-2. This stack exhausts filtered air from the west sample gallery hoods. Emission monitoring consists of a record sampler.

- 296-A-3. This stack exhausts filtered air from hoods in the east sample gallery. Emission monitoring consists of a record sampler. 
- 296-A-5A and 296-A-5B. These stacks exhaust filtered air from laboratory hoods. Emission monitoring for each stack consists of a record sampler.

- 296-A-6. This stack exhausts filtered room air from the east sample gallery and U Cell. Emission monitoring consists of a record sampler.

- 296-A-7. This stack exhausts filtered room air from the PUREX west sample gallery and R Cell. Emission monitoring consists of a record sampler.

- 296-A-8. This stack exhausts filtered air from the PUREX Plant Pipe and Operating gallery and the White Room. Emission monitoring consists of a record sampler.

- 296-A-10. This stack exhausts filtered air from storage tunnel No. 2. Emission monitoring consists of a record sampler.

- 296-A-14. This stack exhausts filtered air from the 293-A Off-gas Treatment and Recovery Building (for nitric acid recovery). Emission monitoring consists of a record sampler.

- 296-A-24. This stack exhausts filtered air from $E$ and $F$ Cell vessels to reduce the ammonium nitrate loading on the 291-A-1 filters. Emission monitoring consists of a record sampler.

A.2.3.2 Uranium-TriOxide Plant ( $\mathrm{UO}_{3}$ Plant). Located in the 200 Area is the UraniumTriOxide $\left(\mathrm{UO}_{3}\right)$ Plant, which formerly produced uranium trioxide from PUREX Plant solutions. In 1972 the $\mathrm{UO}_{3}$ Plant was shut down. Later, it was refurbished to process uranyl nitrate hexahydrate from the PUREX Plant, and was restarted in March 1984. The $\mathrm{UO}_{3}$ Plant operated for the final time in June 1993 processing uranyl nitrate hexahydrate into the more stable compound of uranium trioxide. The plant was deactivated immediately after its final run, in 1993. In December 1994 the facility was transferred from WHC to BHI. $\mathrm{UO}_{3}$ no longer has any point source air emissions, all $\mathrm{UO}_{3}$ stacks were blanked off in late 1994.

A.2.3.3 B Plant and Waste Encapsulation Storage Facility. This facility contains two major operating system areas, B Plant and the Waste Encapsulation Storage Facility (WESF). B Plant was designed and used to separate plutonium from spent nuclear fuel. More recently B Plant was reconfigured to remove ${ }^{137} \mathrm{Cs}$ and ${ }^{90} \mathrm{Sr}$ from high-level liquid waste. At WESF, the ${ }^{137} \mathrm{Cs}$ and ${ }^{90} \mathrm{Sr}$ were converted to solid strontium fluoride and cesium chloride, doubly encapsulated, and placed in water-filled storage basins. WESF is used to store the radioactive strontium and cesium capsules. The 221-B Building contains radioactive contamination remaining from previous production campaigns that must be properly managed.

- 291-B-1. This is the B Plant main stack, which exhausts filtered air from the B Plant canyon, a process cell in the 212-B Building, and the 224-B Building. 
Emission monitoring consists of a record sampler, a backup record sampler, and a beta-gamma CAM.

- 296-B-5. This stack exhausts filtered air from the 221-BB Building, which houses the B Plant process condensate and B Plant steam condensate receiver tanks. Emission monitoring consists of a record sampler and a beta-gamma CAM.

- 296-B-10. This stack exhausts filtered air from the 225-B Building, which is also known as WESF. Emission monitoring consists of a record sampler and a beta-gamma CAM.

- 296-B-13. This stack exhausts filtered air from the 221-BF Condensate Effluent Discharge Facility, which houses process condensate retention tanks. Emission monitoring consists of a record sampler and a beta-gamma CAM.

A.2.3.4 AR and CR Vaults. The AR and CR Vaults are retention and treatment facilities for high-level radioactive liquid wastes.

- 296-A-12. This stack exhausts filtered air from the 244-AR Vault vessel ventilation system. Emission monitoring consists of a record sampler and a beta-gamma CAM. This stack did not operate in 1995.

- 296-A-13. This stack exhausts filtered air from the 244-AR Vault canyon and cells. Emission monitoring consists of a record sampler and a beta-gamma CAM. This stack did not operate in 1995.

- 296-C-5. This stack exhausts filtered air from the 244-CR Vault Cell and vessel ventilation. Emission monitoring consists of a record sampler and a beta-gamma CAM.

A.2.3.5 200 East Area Tank Farms (excluding the Evaporator). Radioactive waste stored in tank farms consists of sludge and saltcake in single-shell tanks (SSTs) and slurry in double-shell tanks (DSTs).

- 296-A-17 and 296-P-26. These stacks exhaust filtered, incondensable vapors from waste storage tanks in the 241-AY and -AZ Tank Farms. Emission monitoring for each stack consists of a record sampler, two silver-zeolite cartridge samplers for volatile radionuclides, and a beta-gamma CAM. The 296P-26 stack serves as the backup exhauster for the 296-A-17 stack.

- 296-A-18. This stack exhausts filtered air from the 241-AY-101 tank annulus. Emission monitoring consists of a record sampler. This stack has been inactive since September 1991. 
- 296-A-19. When this stack was operational, filtered air was released through it from the 241-AY-102 tank annulus. Emission monitoring consisted of a record sampler. This stack did not operate in 1995.

- 296-A-20. 241-AZ Tank Annuli Exhaust. This stack exhausts filtered air from the 241-AZ-101 and -102 tank annuli. Emission monitoring consists of a record sampler.

- 296-A-25. This stack exhausts filtered air from the catch tank at the 244-A lift station. Emission monitoring consists of a record sampler and a beta-gamma CAM.

- 296-A-26. This stack exhausts filtered air from the waste unloading room and sump tank at the 204-AR tank. Emission monitoring consists of a record sampler and a beta-gamma CAM.

- 296-A-27. This stack exhausts filtered air from all 241-AW tanks. Emission monitoring consists of a record sampler, two silver-zeolite cartridge samplers for volatile radionuclides, and a beta-gamma CAM.

- 296-A-28. This stack exhausts filtered air from all tank annuli in the 241-AW tank farm. Emission monitoring consists of a record sampler and a beta-gamma CAM.

- 296-A-29. This stack exhausts filtered air from all 241-AN tanks. Emission monitoring consists of a record sampler and a beta-gamma CAM.

- 296-A-30. This stack exhausts filtered air from all tank annuli in the 241-AN tank farm. Emission monitoring consists of a record sampler and a beta-gamma CAM.

- 296-A-40. This stack exhausts filtered air from all 241-AP tanks. Emission monitoring consists of a record sampler, two silver-zeolite cartridge samplers for volatile radionuclides, and a beta-gamma CAM.

- 296-A-41. This stack exhausts filtered air from all tank annuli in the 241-AP tank farm. Emission monitoring consists of a record sampler and a beta-gamma CAM.

- 296-B-28. This stack exhausts filtered air from the 244-BX saltwell receiver tanks and annulus. Emission monitoring consists of a record sampler, a beta-gamma CAM for the tank exhaust, and a beta-gamma CAM for the annulus exhaust. 
- 296-P-16. A portable exhauster emits filtered air via this stack from the 241C104, 241C105, and 241C106 tanks. Emission monitoring consists of a record sampler and a beta-gamma CAM.

- 296-P-17. This portable exhauster emits filtered air from the 241A104, 241A105, and 241A106 tanks. Emission monitoring consists of a record sampler and a beta-gamma CAM. This stack did not operate in 1995 .

- 296-P-32, 296-P-33, and 296-P-34. These emission points are the portable exhausters for the Rotary Mode Core Samplers. These emission sources exhaust filtered air from the waste tanks being core sampled. Emissions monitoring consists of a record sampler, a beta-gamma CM, and an alpha CAM.

A.2.3.6 200 East Area Evaporator. The evaporator processed liquid waste in 1995. The evaporator is used to remove most of the water from high-level radioactive liquid waste, leaving a slurry that is pumped back to tank farms.

- 296-A-21. This stack exhausts filtered air from the 242-A EvaporatorCrystallizer Building. Emission monitoring consists of a record sampler, an alpha CAM, and a beta-gamma CAM.

- 296-A-22. This stack exhausts filtered air from the 242-A EvaporatorCrystallizer vessel-ventilation system. Emission monitoring consists of a record sampler and a silver-zeolite cartridge sampler for volatile radionuclides, an alpha CAM, and a beta-gamma CAM.

A.2.3.7 Grout Treatment Facility. This facility solidified low-level waste for disposal in underground vaults. The facility was placed in cold standby in 1993.

- 296-G-1. When this stack operates air is exhausted from the GTF Air Filtration Module. Emission monitoring consists of a record sampler and a beta-gamma CAM. This stack did not operate in 1995.

A.2.3.8 209-E Critical Mass Laboratory. The Building was originally designed and used for testing critical mass configurations. Currently the building is being used for office space.

- 296-P-31 (formerly 209-E). This stack exhausts filtered building ventilation air. The particulate alpha and beta radioactivity emissions, from the building exhaust, are sampled with a record sampler.

A.2.3.9 S Plant. S Plant is another name for the Reduction-Oxidation Plant (REDOX), which is the 202-S building. The REDOX Plant was operated as a fuel-reprocessing facility until 1967 when it was shut down. 
- 291-S-1. The REDOX main stack exhausts filtered air from the REDOX canyon, vessel ventilation, and treated dissolver off-gas system. Emission monitoring consists of a record sampler and a beta-gamma CAM.

- 296-S-2. This stack exhausts filtered air from REDOX north and south sample galleries, hoods, and product removal cage. Emission monitoring consists of a record sampler.

- 296-S-4. This stack exhausts filtered air from the REDOX decontamination room and regulated shop and unfiltered air from the regulated tool room, low-level decontamination sink, and special work permit lobby. Emission monitoring consists of a record sampler.

- 296-S-6. This stack exhausts air from the REDOX Plant, the silo gallery, an organic feed tank, and a sample elevator. Emission monitoring consists of a record sampler.

- 296-S-7W and 296-S-7E. These stacks exhaust filtered air from the 233-S REDOX Product building, the REDOX plutonium-processing greenhouse, and process vessel ventilation and load-out area. Emission monitoring for each stack consists of a record sampler and an alpha CAM. The 296-S-7E stack serves as the backup exhauster for the 296-S-7W.

A.2.3.10 T Plant. T Plant is one of the original fuel reprocessing facilities. The last fuel reprocessed there was in 1956. The 221-T Building is currently used to treat, store, repackage, sample, and verify wastes. The 221-T Building also is used for decontaminating and repairing equipment.

- 291-T-1. This stack exhausts filtered air from 221-T canyon and process ventilation. Emission monitoring consists of a record sampler, a beta-gamma CAM, and an alpha CAM.

- 296-T-13. This stack exhausts filtered air from the 221-T Building and canyon ventilation. Emission monitoring consists of a record sampler and a beta-gamma CAM.

- 296-T-7. This stack exhausts filtered air from the 2706 Building only while decontamination activities are being performed, the remaining times it is turned off. Emission monitoring consists of a record sampler and beta-gamma CAM.

A.2.3.11 TRUSAF. The 224-T Building was originally used to purify plutonium nitrate by the lanthanum fluoride process. After phase-out of the bismuth phosphate plants, the lanthanum fluoride process was no longer needed and the facility remained inactive until the early 1970's. At that time, the 224-T Building was modified for storage of plutonium scrap in liquid and solid forms. In 1984 the facility was selected to house the transuranic waste 
storage and assay (TRUSAF) operation. The TRUSAF operation involves the analysis of transuranic waste to verify compliance with the Waste Isolation Pilot Plant (WIPP) waste acceptance criteria. Those containers meeting WIPP acceptance criteria are stored at 224-T pending shipment to the WIPP.

- 296-T-11. This stack exhausts filtered air from the 224-T Building ventilation through the east part of the plenum. Emission monitoring consists of a record sampler.

- 296-T-12. This stack exhausts filtered air from the 224-T Building ventilation through the west part of the plenum. Emission monitoring consists of a record sampler.

A.2.3.12 U Plant. U Plant was constructed as a fuel reprocessing plant but was never used for that purpose. Instead, it was used to recover uranium from bismuth-phosphate waste and high-level radioactive wastes from tank farms. Currently, it is a retired facility with a few offices and shops still in use.

- 291-U-1. This stack exhausts filtered air from U Plant and 221-U canyon ventilation. Emission monitoring consists of a record sampler and a beta-gamma CAM.

A.2.3.13 Plutonium Finishing Plant (PFP). PFP was constructed to produce plutonium metal from recovered nitrate and plutonium nitrate received from the PUREX Plant. PFP also recovered plutonium, in the form of plutonium nitrate, from plutonium scrap. PFP's current mission is to stabilize and store existing inventory of plutonium compounds.

- 291-Z-1. This stack exhausts filtered air from the 234-5Z, 236-Z, and 242-Z Buildings. Emission monitoring consists of a record sampler and an alpha CAM.

- 296-Z-3. This stack exhausts filtered air from $241-Z$ vault sump and vessel ventilation. Emission monitoring consists of a record sampler and an alpha CAM.

- 296-Z-5. This stack exhausts filtered air from $2736-Z B$, the shipping and receiving building. Emission monitoring consists of a record sampler and an alpha CAM.

- 296-Z-6. This stack exhausts filtered air from the 2736-Z Building and its plutonium storage vault ventilation system. Emission monitoring consists of a record sampler and two alpha CAMs. One alpha CAM is located on each of the two ducts feeding into this stack. 
- 296-Z-14. This stack exhausts filtered air from the 232-Z Incinerator Building. Emission monitoring consists of a record sampler and two alpha CAMs.

- 296-Z-15. This stack exhausts filtered air from the 243-Z Low Level Waste Treatment Facility. Emissions monitoring consists of a record sampler.

A.2.3.14 200 West Area Tank Farms (Excluding Evaporators). Radioactive waste stored in tank farms consists of sludge and saltcake in SSTs and liquid and slurry in DSTs.

- 296-P-22. This stack exhausts filtered air from annuli in the 241-SY-101, -102, and -103 tanks. Emission monitoring consists of a record sampler and a beta-gamma CAM.

- 296-P-23 (296-P-28 backup stack). This stack exhausts filtered air from the 241-SY-101, -102, and -103 tanks. Emission monitoring consists of a record sampler and a beta-gamma CAM

- 296-S-15. This stack exhausts filtered air from tanks 241-SX-107 through 112 and 241-SX-114. Emission monitoring consists of a record sampler and a beta-gamma CAM.

- 296-S-22. This stack exhausts filtered air from the 244-S saltwell receiver tank and annulus. Emission monitoring consists of a record sampler, an alpha CAM, and a beta-gamma CAM.

- 296-T-18. This stack exhausts filtered air from the 244-TX saltwell receiver tank and annulus. Emission monitoring consists of a record sampler, an alpha CAM, and a beta-gamma CAM.

A.2.3.15 200 West Area Evaporators. The evaporators are designed to remove most of the water from radioactive liquid waste; the resulting slurry is then routed to tank farms for storage. The 242-T Evaporator-Crystallizer has been deactivated and has been inoperable since 1986. The 242-S Evaporator-Crystallizer did not operate in 1995, but is on standby.

- 296-S-18. This stack exhausts filtered air from the 242-S Evaporator-Crystallizer Building. Emission monitoring consists of a record sampler and a beta-gamma CAM.

- 296-T-17. This stack exhausts filtered air from the 242-T EvaporatorCrystallizer and cold-cell ventilation. Emission monitoring consists of a record sampler, an alpha CAM, and a beta-gamma CAM. 
A.2.3.16 222-S Analytical Laboratories. The 222-S Analytical Laboratories provide chemical and radiochemical analytical support for WHC, including environmental and effluent sample analysis, research and development, and tank farm waste characterization.

- 296-S-16. This stack exhausts filtered air from the 219-S Building and waste tanks. Emission monitoring consists of a record sampler.

- 296-S-21. This stack exhausts filtered air from 222-S Laboratory hoods, gloveboxes, hot-cells, and room ventilation system. Emission monitoring consists of a record sampler, an alpha CAM, and a beta-gamma CAM.

A.2.3.17 Waste Sampling and Characterization Facility (WSCF). The WSCF laboratory provides low-level radiological and chemical analyses on various types of samples and sample medias. The majority of the analyzed samples are used to determine compliance with the requirements of environmental regulations and U.S. Department of Energy (DOE) Orders. Note that WSCF is technically located in the 600 Area, and is immediately outside of the 200 West Area's perimeter. It is included in the section on for 200 West Area, since its releases are modelled from the 200 West Area.

- 696-W-1. This stack exhausts filtered air from the analytical laboratory located on the main floor of the 6266 building. Emission monitoring consists of a record air sampler.

- 696-W-2. This stack exhausts filtered air from the Nuclear Spectroscopy Laboratory located in the basement of the 6266 building. Emission monitoring consists of a record sampler.

A.2.3.18 Waste Verification \& Sampling Facility. The facility is used to verify the contents of drums received from generators. Because of limited use, it was transferred to West Tank Farms in 1995.

- 296-W-3. This exhaust discharges filtered air from the 213-W building. Emission monitoring consists of a record sampler and a beta-gamma CAM.

\section{A.2.4 300 Area Facilities}

The 300 Area consists primarily of laboratories, research facilities, and a steam plant. Emission points in the 300 Area are shown in Figure 1-6.

A.2.4.1 304 Uranium Concretion Facility. In 1994, this facility was operated in a limited capacity for preparing radioactively contaminated sludges and fines for disposal or storage. Activities performed at this facility included mixing radioactive contaminated matrices with cement and water. The facility is currently in transition to closure under RCRA regulations. Its stack, 304-CON-EX, was sealed off in 1995 and no longer has an emission point. 
A.2.4.2 306-E Metal Fabrication Development Building. This building is in use for materials testing and fabrication. In the past, this building housed three separate operations; (1) a large, high-bay used for unfueled test article fabrication for FFTF, (2) nondestructive, radiography examination in a series of shielded cells, and (3) a small depleted-uraniumpowder laboratory used to fabricate insulator pellets for the FFTF fuel pins. In August 1995, this facility was shutdown and has since been stabilized, changing its radiological status to a fixed contamination area.

- 306-E-ULAB. This stack exhausts filtered air from the Uranium Oxide Laboratories in rooms 158 and 159 , which no longer contains loose contamination. Emission monitoring consists of a record sampler.

A.2.4.3 308 Fuels Development Laboratory. The 308 Laboratory was used for fabricating mixed-oxide fuel pins used by FFTF. The facility houses a shutdown swimming-pool-type Test Reactor and Isotope Production reactor by General Atomics (TRIGA) that was used in neutron radiography of the completed pins. The 308 Building is in transition to shutdown.

- 308-TRIGA. This stack discharges filtered air from the reactor hall housing the TRIGA reactor. The reactor, however, is permanently shut down and partially defueled. Emission monitoring consists of a particulate record sampler.

A.2.4.4 309 Plutonium Recycle Test Reactor. The 309 Building's containment dome and support facilities once housed the Plutonium Recycle Test Reactor (PRTR). In 1962, the Plutonium Recycle Critical Facility (PRCF) was added to support the PRTR operations. By 1975 , the PRTR was deactivated, all of the fuel removed from the building, and the fuel storage basin decontaminated. In the mid 1980's, an extensive cleanout effort removed most of the process equipment and vessels. The ground level of the containment dome is currently being used an assembly shop and the remainder of the building is used as office space.

- 309-PRTR. The 309 Building's primary stack ventilates what used to be the PRTR facility. Periodic confirmatory measurements are used to confirm that emissions are at or below acceptable levels.

- 309-RTF-EX. 309 Building rupture loop annex exhaust stack. Periodic confirmatory measurements are used to confirm that emissions are at or below acceptable levels. 
A.2.4.5 340 Complex. The 340 Complex houses the radioactive liquid waste and solid waste handling operations for the 300 Area. The 340-A Building contains six aboveground storage tanks for radioactive liquid waste. The east side of the 340-B building is a railway car loadout facility for shipping liquid waste to the 200 Areas. The west side of the 340-B building is a storage area for non-radioactive and radioactive solid waste.

- 340-NT-EX. This stack exhausts filtered air from the 340 Building vault, the 340 Building vault tanks, and the 340-A Building waste tanks. Emission monitoring consists of a particulate record sampler, an alpha CAM, a betagamma CAM, and a record sampler for volatile ${ }^{131} \mathrm{I}$.

- 340-B-BLDG. This stack exhausts filtered air from the 340-B East Building. The stack exhaust system operates when railway cars are housed within the facility. Emissions monitoring consists of a particulate record sampler.

- 340-DECON. This stack exhausts air from the 340 Facility truck lock, operator's office, change rooms, decontamination area, and sampling hood. Emissions monitoring consist of a particulate record sampler.

A.2.4.6 377 Geotechnical Engineering Laboratory. This facility was formerly known as the 377 Steam Generator Examination Facility. In 1993 the facility was renamed the 377 Geotechnical Engineering Laboratory to reflect the change in operations. The stack name was also changed from $377-$ SGEF to $377-$ GEL. The facility was formerly used for studying a steam generator that had been removed from a nuclear power plant. The steam generator was removed before 1990 and the facility was decontaminated. In 1991 and 1992, the facility was modified for use as a physical properties testing laboratory for radioactively contaminated soils and began laboratory operation in 1993.

- 377-GEL. This stack exhausts filtered building ventilation air. The exhaust is sampled for particulate alpha and beta radioactivity.

\section{A.2.5 400 Area Facilities}

The 400 Area consists of the FFTF, the Maintenance and Storage Facility (MASF), and the Fuels Materials Examination Facility. Emission points in the 400 Area are shown in Figure 1-7.

A.2.5.1 Fast Flux Test Facility. FFTF, located in the 400 Area, is a 400 -megawatt thermal, sodium-cooled, low-pressure, high-temperature reactor plant. It was used for irradiation testing of breeder reactor fuels and materials.

- FFTF-RE-SB. Lower Reactor Service Building (RSB). This exhaust discharges unfiltered air from the lower level of the RSB. Emission monitoring consists of 
a particulate record sampler, a beta CAM, a noble gas monitor, and a record sampler for volatile ${ }^{131} \mathrm{I}$.

- FFTF-CB-EX. Combined exhaust. This stack exhausts normally unfiltered air from the reactor containment and gases from the argon processing system. Standby particulate filters are automatically dampered into the system if airborne radioactive particulate concentrations exceed administrative limits. Emission monitoring consists of an particulate record sampler, a beta CAM, a noble gas monitor, a tritium (HTO) record sampler, and a record sampler for volatile ${ }^{131} \mathrm{I}$.

- FFTF-HT-TR. Heat Transport System South. This exhaust discharges normally unfiltered air from portions of FFTF that are exterior to the containment. Standby particulate filters are automatically dampered into the system if airborne radioactive particulate concentrations exceed administrative limits. Emission monitoring consists of a record sampler, a beta CAM, and a noble gas monitor.

\begin{abstract}
A.2.5.2 437 MASF. The Maintenance and Storage Facility (MASF) is a multipurpose service center supporting the specialized maintenance and storage requirements of FFTF. The MASF provides the capability for sodium film removal, decontamination, repair, and storage of nonfueled components and hardware for FFTF.
\end{abstract}

- 437-MN\&ST. This exhaust discharges filtered air from the MASF. Emission monitoring consists of a record sampler and a beta CAM.

- 437-1-61. This exhaust discharges filtered air from the MASF. Emission monitoring consists of a record sampler and a beta CAM. 
WHC-EP-0527-5

\section{APPENDIX B}

MONITORING PROGRAM FOR RADIOACTIVE AIR EMISSIONS AND LIQUID EFFLUENTS

B-1 
TYM MAR TTENTIONALLY LE: BLANK

III:" - rimmer 


\section{APPENDIX B}

\section{MONITORING PROGRAM FOR AIR EMISSIONS AND LIQUID EFFLUENTS}

The WHC monitoring program for air emissions and liquid effluents comprises several activities designed to obtain and report high-quality sampling and analysis data to determine compliance with U.S. Department of Energy guidelines and federal, state, and local regulations. This program includes facilities monitored by BHI, since BHI has contracted WHC to perform effluent monitoring and reporting.

\section{B.1 AIR EMISSION SAMPLE COLLECTION AND ANALYSIS}

A representative sample of air emissions during the collection period is obtained by extracting the sample at a velocity that is as close as practicable to the stack airflow velocity and by holding the ratio of sample volume to total stack discharge volume constant within $\pm 20 \%$.

The following methods are generally used to sample air emissions.

- Record Sampler. A stack record sampler collects radioactive emissions on a collection media, usually a particle filter, that is exchanged and evaluated as specified by WHC Air and Water Services. Particulate record samples are analyzed by the receiving laboratory for total alpha and total beta activity. Record samplers provide an indication of the amounts and concentrations of airborne radioactive material being discharged to the environment. Collection media for record samplers may vary, and is dependent on the radionuclide and chemical form of the substance being sampled. Record samplers do not utilize detectors, strip charts, nor alarms.

- Continuous Air Monitor. Continuous air monitors (CAMs) serve as warning devices to alert personnel to air emission releases exceeding normal operating parameters. A CAM collects particles on a filter monitored continuously by a radiation detector. The CAM filter may also be used as a backup for the record sample if necessary.

The following are the sample collection media utilized for collecting record samples.

- Particle Filters. Particle filters are the most common collection media used at Hanford to collect radioactive airborne particles. Filter medias for routine samples usually consist of either a membrane filter or glass fiber filter.

- Silver-Zeolite or Charcoal Cartridges. Silver-zeolite or charcoal cartridges are used to collect iodine and volatile forms of antimony, ruthenium, and tin. 
- Silica-Gel. Silica-gel columns are used to collect tritium $\left({ }^{3} \mathrm{H}\right)$.

- Grab Air Samples. Grab air samples are used to collect samples of noble gases (e.g. Argon-41). A known volume of gas is collected with a sampler and then usually analyzed using a gamma-ray detector.

The PUREX and PFP facilities routinely report Plutonium-241 emissions. These emissions are calculated from facility inventories, since it can not be readily sampled and analyzed using conventional methods.

\section{B.2 LIQUID EFFLUENT SAMPLE COLLECTION AND ANALYSIS}

Liquid effluent sample collection schemes are designed to provide a representative sample of the effluent. Volume proportionality and extraction from a well-mixed stream are accomplished where practicable. Liquid effluent streams are sampled using the following: (1) weekly grab sampling, (2) batch sampling for a retention area, (3) incremental timeproportional sampling, (4) incremental flow-proportional sampling, and (5) continuous flowproportional sampling.

Samples of the liquid effluent are collected for one month, composited, and then analyzed for total alpha and total beta activity. Specific radionuclide analyses are also performed on liquid effluent streams with a potential to exceed predetermined limits or as specified by WHC Effluent Monitoring.

\section{B.3 DATA HANDLING}

Radionuclide air and liquid effluent data received, are as follows:

- $\quad$ Stack record air sampler flowrates, total flow volume, and sample times.

- Stack flow rates determined via 40 CFR 60 Appendix A Methods 2 and $2 \mathrm{~A}$ or employing mass flowmeters on the stack, or an approved alternate method.

- $\quad$ Liquid discharge volumes.

- Sample analysis data.

These data are reviewed continually for consistency and errors. Concentration values calculated weekly, monthly, and quarterly are compared with past and present facility releases and with environmental release guides. All anomalies are investigated.

Generally, total alpha and total beta analysis results do not accurately reflect specific radionuclide emissions. However, if alpha results indicate a release concentration much less than the most restrictive limit for a specific alpha emitting radionuclide, the discharge is assumed to be within the release limits for all alpha emitters. The same methodology is employed for beta emitters. Exceptions to this methodology do exist. In liquids, results for 
total beta do not include ${ }^{3} \mathrm{H}$, a low-energy beta emitter, and volatile alpha and beta emitters that may be driven off with evaporation (e.g., ${ }^{129} \mathrm{I}$ and ${ }^{210} \mathrm{Po}$ ). Radioactive gases and volatile radionuclides are not included in total alpha and beta analyses of air samples. Separate analyses are performed for ${ }^{3} \mathbf{H}$, volatile substances, and gases when appropriate. 
THIS PACE INTENTTONALLY LEH' DLANK 
WHC-EP-0527-5

\section{DISTRIBUTION}

Number of Copies

\section{OFFSITE}

U.S. Environmental Protection Agency, Region 10

1200 Sixth Avenue

Seattle, WA 98101

G. M. Leitch (5)

AT-081

A. Frankel

R. W. Poeten

AT-081

6

Washington State Department of Health

Air Emissions and Defense Waste Section

Division of Radiation Protection

P. O. Box 47827

Olympia, WA 98504-7827
A. W. Conklin (5)

J. E. Erickson

1

Washington State Department of Ecology

M. A. Wilson

B5-18

5

U.S. Department of Energy-Headquarters

R. F. Pelletier (3)

EH-232

M. K. Harmon

EM-442

K. C. Duvall

$\mathrm{EH}-232$

Confederated Tribes of the Umatilla Indian Reservation

P. O. Box 638

Pendleton, OR 97801

J. R. Wilkinson

Distr-1 


\section{DISTRIBUTION (continued)}

\section{Number of Copies}

$\underline{\text { OFFSITE }}$

1

1

\section{$\underline{\text { ONSITE }}$}

10

14
Nez Perce Tribe

Environmental Restoration and Waste Management

P. O. Box 365

Lapwai, ID 83540

D. L. Powaukee

Yakama Indian Nation

Environmental Restoration Waste Management Program

P.O. Box 151

Toppenish, Washington 98948

R. Jim

$\underline{\text { Richland Operations Office }}$

G. M. Bell

A5-52

R. F. Brich

H0-12

J. B. Hall

A5-15

J. E. Mecca

R3-81

B. M. Pangborn

A5-55

J. E. Rasmussen

A5-15

H. M. Rodriguez

A5-15

D. C. Ward

A5-15

A. H. Wirkkala

A5-11

DOE-RL Public Reading Room

$\mathrm{H} 2-53$

Pacific Northwest National Laboratory

M. Y, Ballinger

P7-68

E. G. Damberg

P7-68

R. L. Dirkes

K6-75

W. T. Farris

K3-54 
WHC-EP-0527-5

DISTRIBUTION (continued)

Number of Copies

ONSITE

Pacific Northwest National Laboratory (continued)

R. W. Hanf, Jr.

K6-75

G. R. Hoenes

P7-79

S. J. Jette

P7-68

T. J. Lazarski

P7-79

T. W. Moon

P7.79

K. Rhoads

K3-54

M. J. Sula

P7 68

H. T. Tilden II

P7-79

R. K. Woodruff

P7-68

PNNL Reference Library

10

Bechtel Hanford. Inc.

E. T. Coenenburg

H9-11

R. G. Egge

T7-05

M. E. Greenidge

$\mathrm{X} 5-54$

J. T. Hadley

H9-11

S. J. Ingle

H9-12

R. J. Landon

H0-18

L. A. Mihalik

H9- 12

M. R. Morton

$\mathrm{X} 5-53$

D. W. Long

T7-05

J. P. Zoric

X5-57

153

Westinghouse Hanford Company

President's Office

B3-01

D. J. Alexander

S3-24

D. Alison

R1-51

C. P. Allen

R1-51

D. D. Bachand

R1-51

J. M. Barnett

T4-08

J. A. Bates

H6-36

R. J. Boom

T6-12

M. J. Brown

S6-71

K. R. Busching

T4-03 
WHC-EP-0527-5

\section{DISTRIBUTION (continued)}

\section{Number of Copies}

\section{$\underline{\text { ONSITE }}$}

Westinghouse Hanford Company (continued)

G. J. Carter, Jr.

$\mathrm{X} 5-53$

S. E. Campbell

$\mathrm{T} 4-03$

B. C. Cornwell

L5-70

J. F. Brown

S5-03

G. M. Crummel

R1-51

N. R. Dahl

N2-57

W. E. Davis

H6-36

J. L. Demarest

G1-40

L. P. Diediker (75)

H6-36

A. J. Diliberto

H6-10

T. A. Dillhoff

N2-57

B. J. Dixon

B4-20

W. T. Dixon

H6-21

J. J. Dorian

H6-30

D. L. Dyekman

T4-08

D. L. Edwards

S3-27

B. G. Erlandson

R2-36

D. J. Farquhar

H7-34

D. P. Fasset

T3-28

D. L. Flyckt

S6-71

T. P. Frazier

H6-25

R. G. Gant

X3-79

L. A. Garner

R2-36

C. K. Girres

T3-28

B. P. Gleckler

H6-36

F. T. Green

H6-29

E. M. Greager

H6-36

P. R. Gunter

R1-52

R. D. Gustavson

R2-50

K. J. Haggerty

L6-04

D. L. Halgren

L6-04

M. J. Hall

T6-12

M. B. Jaeger

L4-02

A. R. Johnson

H6-20

D. L. Johnson

S5-66

R. E. Johnson

H6-25 
WHC-EP-0527-5

DISTRIBUTION (continued)

Number of Copies

ONSITE

Westinghouse Hanford Company (continued)

S. E. Killoy

S4-66

J. M. Kisielnicki

L6-39

G. J. LeBaron

S6-19

L. L. Lockrum

S3-90

J. J. Luke

C. C. Macinnis

H6-25

B. M. Markes

B3-28

C. E. Marple

H6-20

B. A. Mayancsik

S4-56

D. J. McBride

T4-03

P. C. Miller

D. L. Mitchell

T5-54

R1-51

J. A. Morrison

H6-29

S. E. Myers

S3-31

S. T. Noga

S3-24

S5-66

J. K. Perry

A. D. Poor

H6-25

D. E. Rasmussen

L6-55

J. H. Rasmussen

N1-47

T. M. Ridge

S6-51

S4-66

J. W. Schmidt (2)

H6-20

P. J. Sullivan

T5-54

R. W. Szelmeczka

L6-05

L. W. Vance

B1-13

E. F. Votaw

G1-13

D. J. Watson

X3-79

L. L. Weaver

T6-12

C. D. Wollam

S6-22

M. T. York

H6-29

Central Files

A3-88

Correspondence Control

A3-01

DPC

A3-89 
WHC-EP-0527-5

This page intentionally left blank.

Distr-6 